NASATECHNICAL

MEMORANDUM
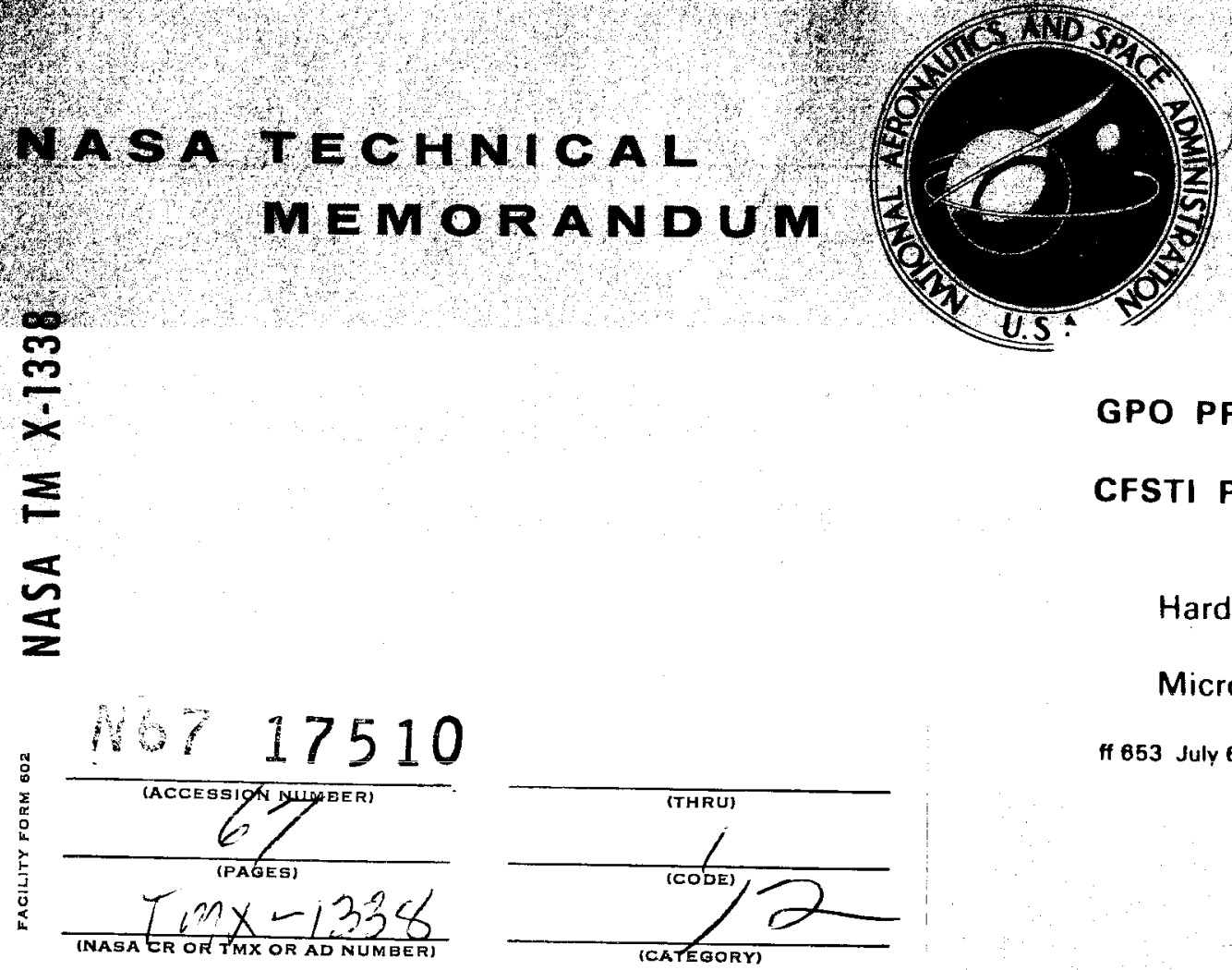

NASA TM X-1338:

GPO PRICE \$

CFSTI PRICE(S) \$

I.

Hard copy (HC)

Microfiche (MF). .5

ff 653 July 65

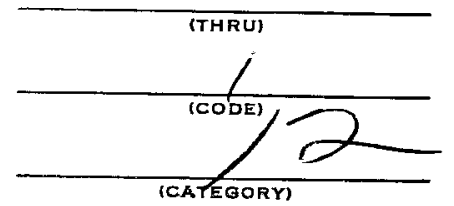

MERCURY DROPLET SIZE

AND DISTRIBUTION IN GLASS

CONDENSER TUBE IN 1.g AND

ZERO-GRAVITY ENVIRONMENTS

by Jonas I. Sturas

Lewis Research Center

Cleveland, Obio

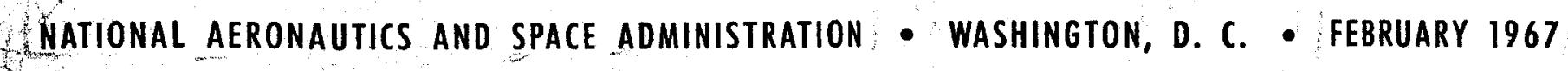


NASA TM X-1338

MERCURY DROPLET SIZE AND DISTRIBUTION IN GLASS CONDENSER

TUBE IN 1-g AND ZERO-GRAVITY ENVIRONMENTS

By Jonas I. Sturas

Lewis Research Center

Cleveland, Ohio 
SUMMARY ................................. 1

INTRODUCTION . . . . . . . . . . . . . . . . . . . . . . . 1

APPARATUS AND PROCEDURE . . . . . . . . . . . . . . . . . . . 2

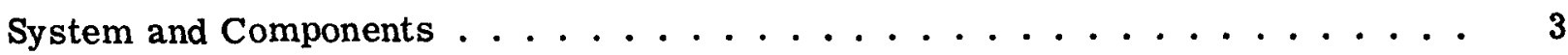

Instrumentation. .......................... 8

Test Procedure. . . . . . . . . . . . . . . . . . . . . . 9

RESULTS AND DISCUSSION. . . . . . . . . . . . . . . . . . . . . 9

General Description of Film Observations . . . . . . . . . . . . . . 9

Analysis ............................ 10

Basic model. . . . . . . . . . . . . . . . . . . . . 11

Droplet size distribution . . . . . . . . . . . . . . . . . 12

Correlation of Parameters a, b, c, and $\delta_{\mathrm{mf}}$ With System Parameters . . . . 37

Ground tests. . . . . . . . . . . . . . . . . 37

Airplane tests ......................... . . . . 40

Effect of gravity. . . . . . . . . . . . . . . . . . . . . . . . . 43

Modification of Ground Test Data to Obtain Zero-Gravity Droplet Distribution . . 47

Droplet flux ........................ . . . 47

Ground tests. . . . . . . . . . . . . . . . . . . . 47

Effect of gravity on droplet flux . . . . . . . . . . . . . . . . . . . . . . 48

Maximum stable droplet size . . . . . . . . . . . . . . . . . . 48

Critical droplet size in ground tests . . . . . . . . . . . . . . . . . . . . . 49

Droplet height on condenser wall in ground tests ............. 51

$\begin{array}{lr}\text { CONCLUDING REMARKS } & 51\end{array}$

APPENDIXES

A - SYMBOLS . . . . . . . . . . . . . . . . . . . . . . 53

B - DROPLET SIZE DISTRIBUTION FUNCTION . . . . . . . . . . . . . 55

C - VAPOR VELOCITY DISTRIBUTION ALONG CONDENSER TUBE . . . . . . 58

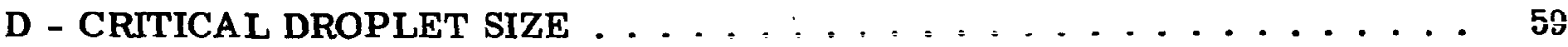

REFERENCES . . . . . . . . . . . . . . . . . . . . . 64 


\title{
MERCURY DROPLET SIZE AND DISTRIBUTION IN GLASS CONDENSER TUBE IN l-g AND ZERO-GRAVITY ENVIRONMENTS
}

\author{
by Jonas I. Sturas
}

Lewis Research Center

\section{SUMMARY}

An experimental study was made of mercury droplet size and flux distribution in a horizontal convectively cooled glass mercury vapor condenser tube in 1-g and zerogravity environments. The film analysis showed that the liquid phase was dispersed throughout the vapor phase in the form of discrete droplets, even in the higher vaporquality range studied. The dispersed droplet breakup and coalescense in two-phase flow occurred simultaneously and continuously along the length of the condenser tube. The observed droplet size distribution was best described by the modified gamma distribution function.

Droplet size and flux distribution obtained from a series of high-speed film measurements, at various velocities and quality conditions along the tube length, were described statistically and fitted to a modified gamma distribution. The numerical values of three characteristic parameters that describe the gamma distribution curve varied with vapor velocity and quality.

The critical droplet size measured from a droplet track left on a condenser wall at its incipient movement and the maximum stable droplet size measured in a turbulent vapor stream were essentially linear functions of vapor quality and vapor velocity.

\section{INTRODUCTION}

The design and development of liquid-metal Rankine cycle electric power generating systems for space flight applications have defined a need for improved methods of predicting pressure drop and heat-transfer characteristics of two-phase flows associated with boiling and condensing processes in variable gravity environments. The condensing process is of particular interest since, in a Rankine turbogenerator system, the conden- 
ser component has stringent limitations on pressure level and pressure drop imposed by the turbine and circulating pump requirements.

The accuracy of analytically derived thermofluid dynamic relations is keyed to the assumption of realistic flow models that accurately characterize the formation, orientation, and transport of the liquid-phase portion of the two-phase flow. It can also be imagined that the flow characterization may be significantly affected by the imposed gravity environment and that the degree of this effect may be different at various flow conditions in a given tube geometry and locally in a given tube at a fixed flow condition.

Prior studies directed toward analytical definition of two-phase frictional pressure drop for mercury vapor condensation in nonwetting condenser tubes have been conducted by Koestal and Gido (ref. 1) and Heller (ref. 2) based on a postulated 1-g flow model.

As part of an overall program conducted at the NASA Lewis Research Center on mercury vapor condensing characteristics in 1-g and zero-gravity environments (ref. 3), a specific study was made to gain a better understanding of flow regimes in a typical condehser tube. The results discussed herein are based on high-speed motion-picture analysis of nonwetting mercury vapor condensing flow regimes in a uniformly cooled glass tube under 1-g and zero-gravity conditions. The analysis includes definition of droplet sizes and frequency distribution, maximum stable drop size, and critical drop size on the condenser tube wall at incipient movement. Empirical relations were derived from measured data for calculating droplet size distribution and other droplet parameters pertinent to two-phase-flow mechanisms.

Available on loan is a Lewis motion picture (C221) that includes an introduction to space power systems, a description of the MECA - AJ-2 flight package and associated hardware, and high-speed film sequences of mercury condensing in 1-g and zero-gravity environments. (A request card is included at the back of the report.)

Experimental data were obtained for a range of mercury weight flow rates from 0.315 to 0.435 pound per second, and the dependence of droplet distribution on vapor velocity and vapor quality was determined by photographic studies at various positions along the condensing length.

\section{APPARATUS AND PROCEDURE}

The experimental apparatus, system components, instrumentation, and test procedures were essentially the same for tests performed on the ground and in the zerogravity flight facility. 


\section{System and Components}

The test apparatus is shown schematically in figure 1, and the system flow diagram is shown in figure 2. The apparatus consisted of an expulsion tank that supplied tripledistilled mercury to the boiler. The boiler, consisting of a preheater and an electrically heated boiling section, produced a continuous supply of mercury vapor at a quality of 90 percent or greater. To assure consistent vapor flow and to minimize liquid carryover, the vapor was passed through a plenum chamber filled with stainless-steel cuttings. The mercury vapor flow was controlled to the desired value by a pressure regulator and a calibrated orifice.

The condensing tubes consisted of 0.440 -inch constant-diameter low-alkali borosilicate glass tubing for the ground experiments and 0.400-inch constant-diameter transparent high-silica glass tubing for the aircraft flights. The overall length of the condenser tube in all cases was 87 inches. The tube was uniformly cooled by a crossflow of nitrogen gas issuing from two diametrically opposed manifolds, as shown in figure 1 . The mercury vapor was condensed completely inside the tube, and the liquid mercury passed through an orifice at the end of the tube into the receiver. The desired condensing pressure was maintained by controlling the receiver cover gas pressure.

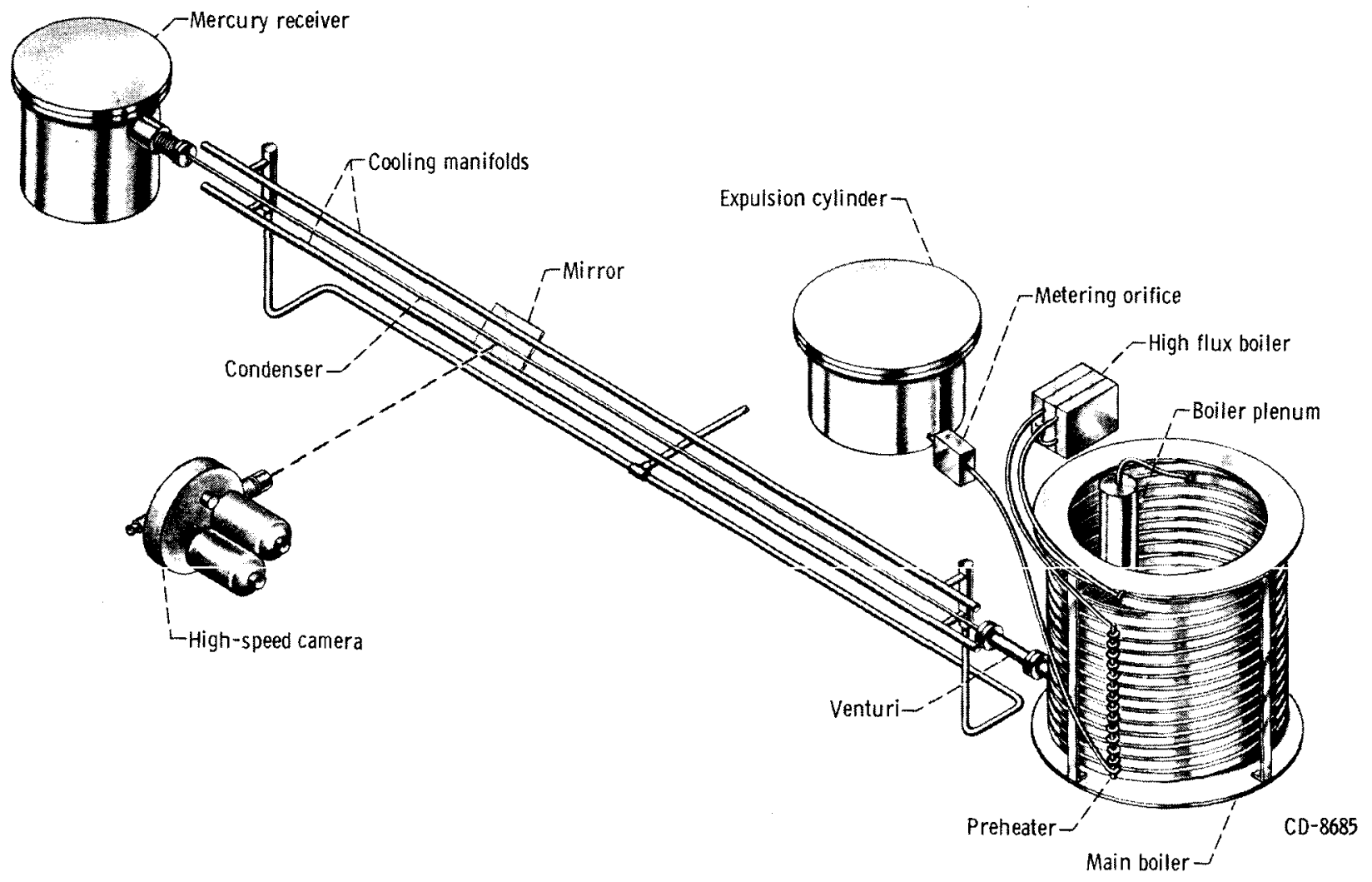

Figure 1. - Schematic drawing of test loop. 


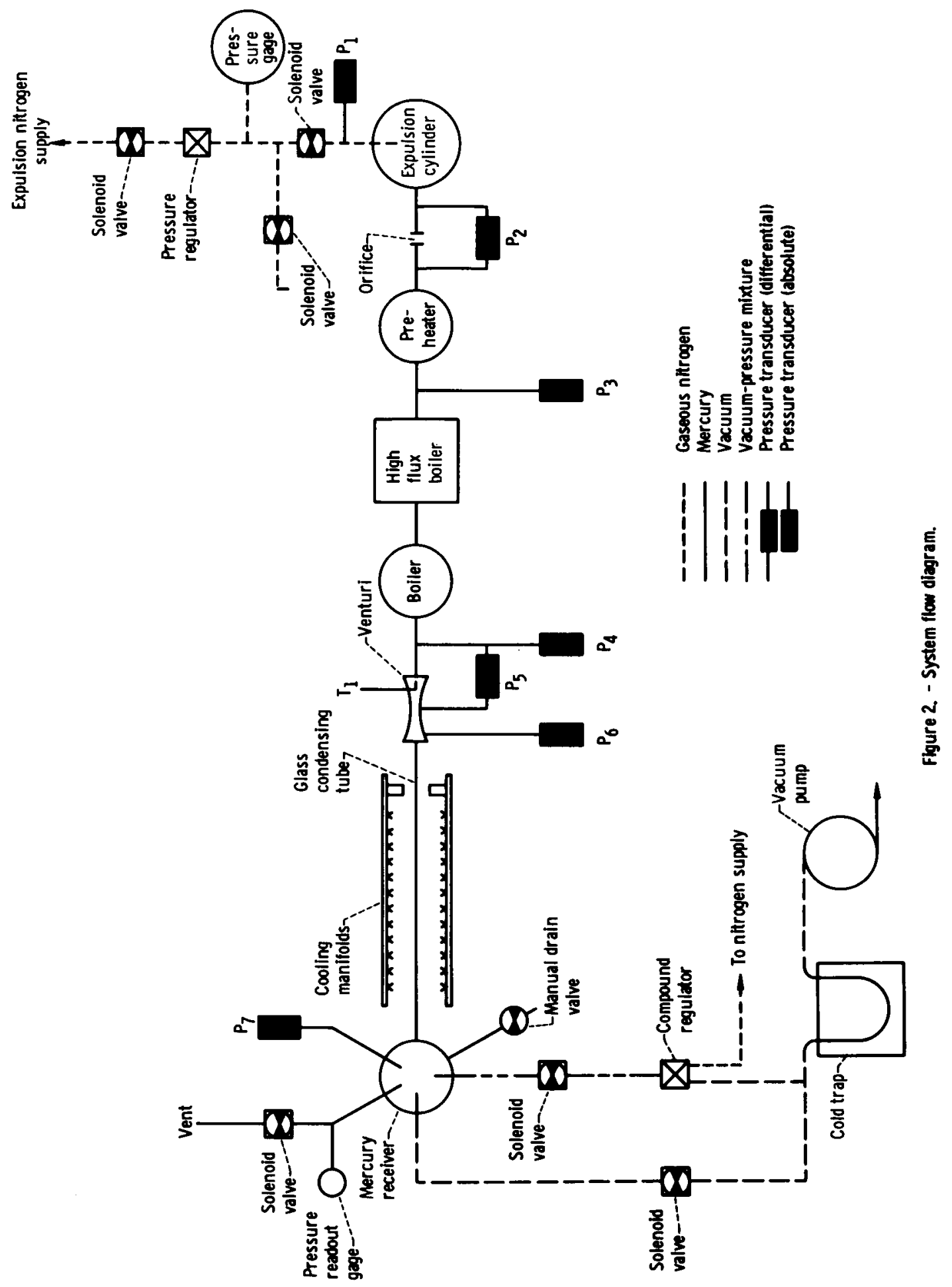




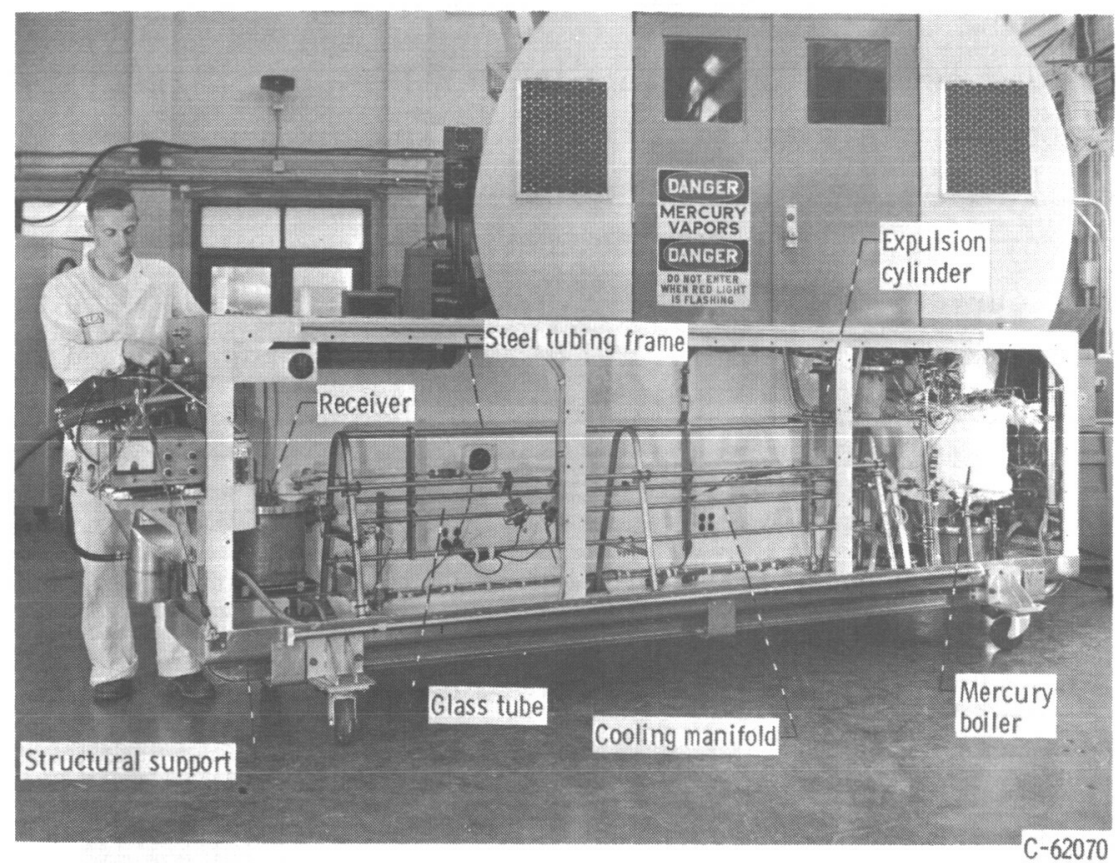

Figure 3. - Basic test system subassembly.

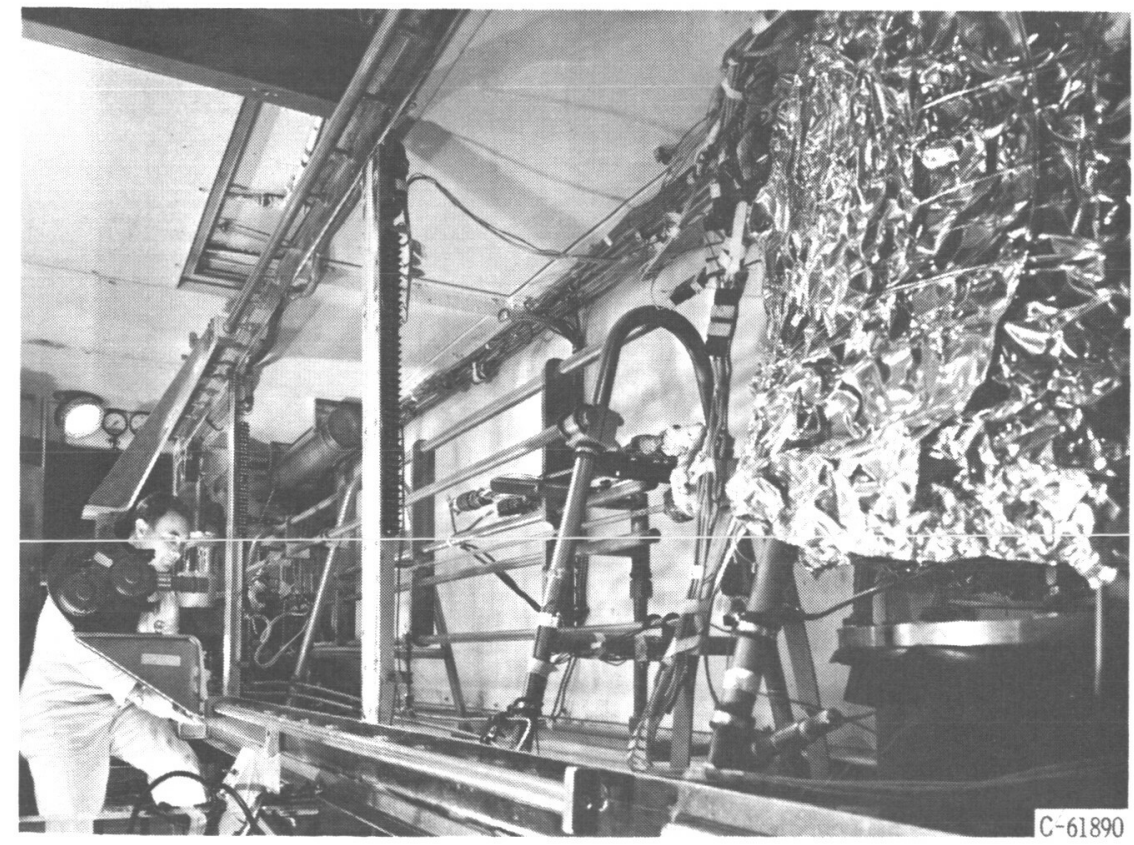

Figure 4. - Experimental system installed in bomb-bay of aircraft. 
The experimental system installed in the flight package enclosure is shown in figure 3. The experimental system installed in the bomb-bay of the AJ-2 aircraft for the zero-gravity flights is shown in figure 4 .

Films of the condensation process were taken with a 16 millimeter high-speed camera at rates ranging approximately from 1000 to 8000 frames per second. Although the initial camera-speed acceleration produced a nonuniform frame rate, the transients involved and the frame rate at any film position could be accurately determined from the curves shown in figure 5. These curves, one for each film taken, were obtained from the measurement of the number of frames between timing marks, which were recorded on the edge of the film in the form of light flashes at the rate of 120 impulses per second, as illustrated in figure 6.

In addition to the high-speed camera, a low-speed camera with a wide-angle lens was used in the aircraft to record the gross movement of the mercury interface during zerogravity maneuvers. The vertical acceleration was indicated by an accelerometer that was in the field of view of the camera. The arrangement of the camera is shown in figure 7.

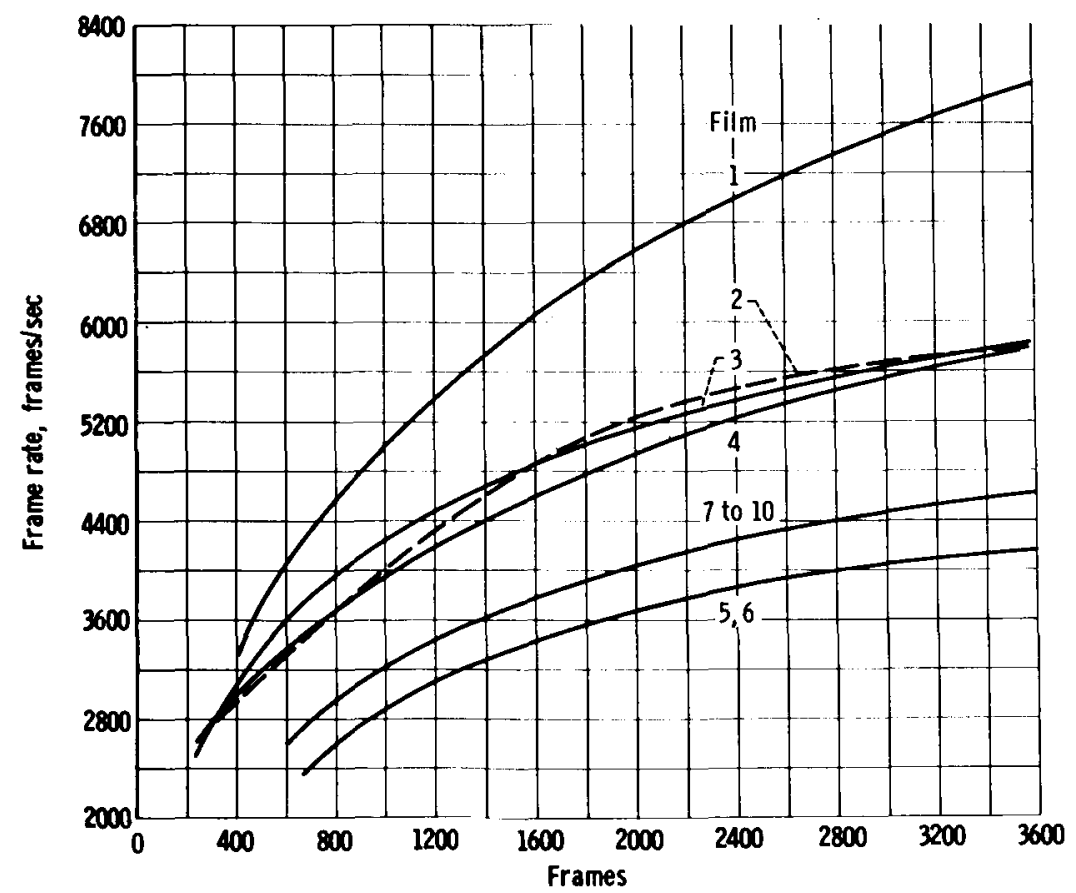

Figure 5. - Frame rate is function of number of frames. Reference point is from frame following last perforated number. 


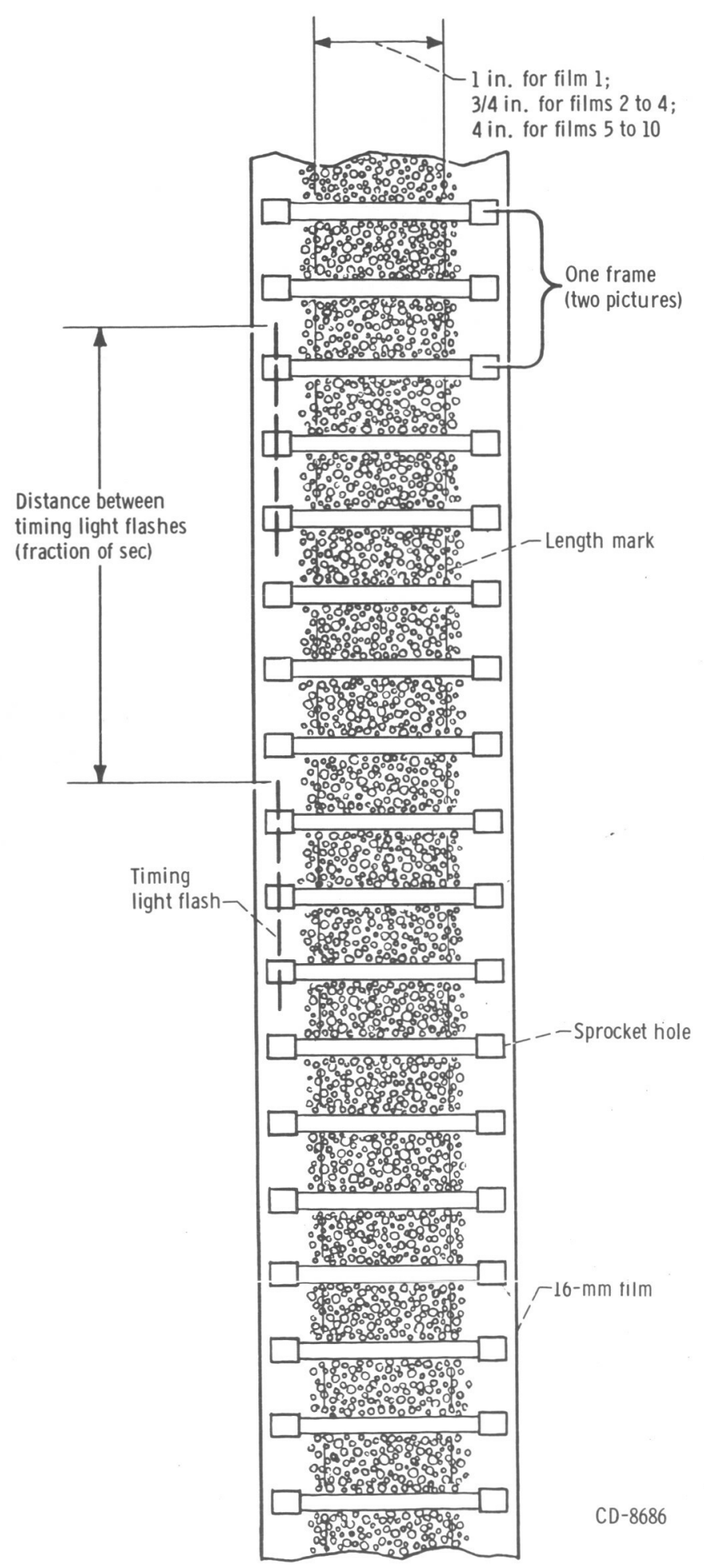

Figure 6. - Schematic drawing of 16-millimeter film showing relation between timing light flashes and frames recorded along film. 


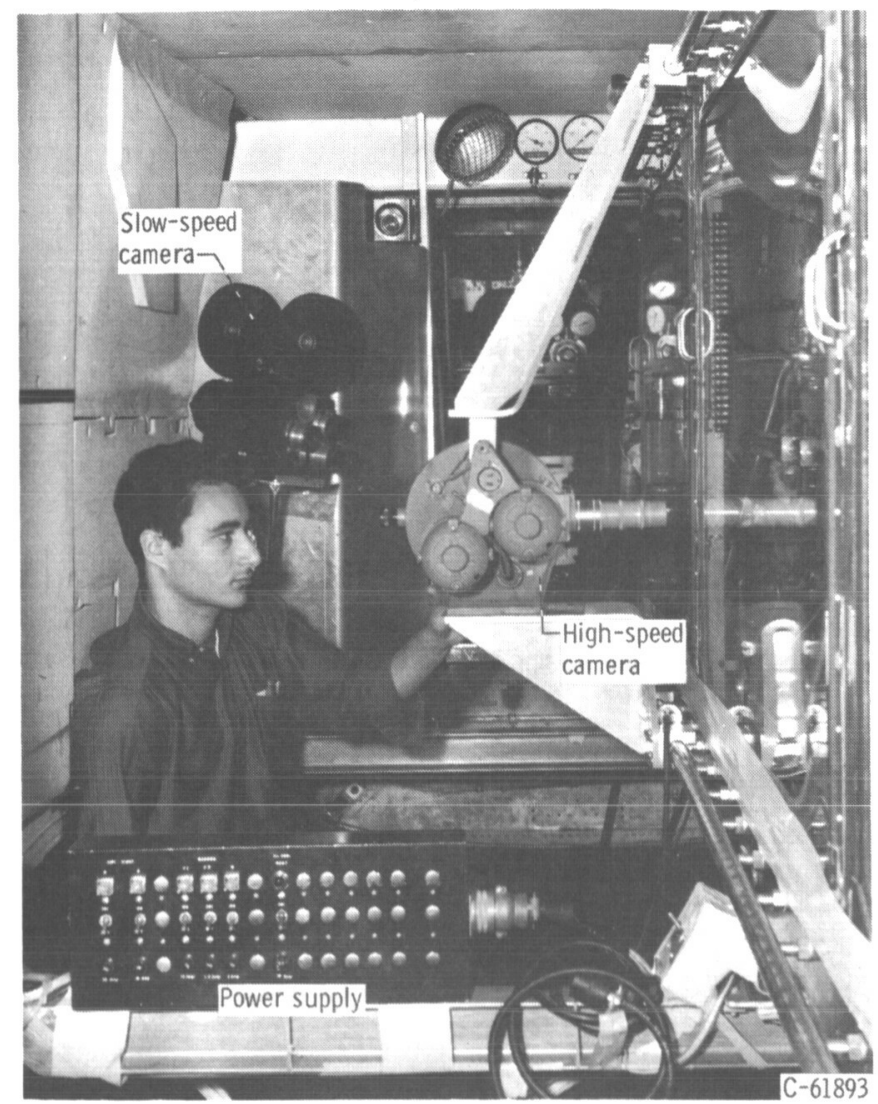

Figure 7. - Arrangement of camera relative to test rig used in airplane experiment.

\section{Instrumentation}

The mercury vapor flow rate into the condensing tube was measured by a venturi with a throat diameter of 0.277 inch and an exit diameter equal to the condenser tube diameter.

Oscillographs were used to record the data in both facilities. In the aircraft, the accelerations generated along the three axes (longitudinal, lateral, and vertical) were sensed by accelerometers located in the bombbay near the geometric center of the experiment. The "g" level indications were used for aircraft control throughout the maneuver and also were recorded to provide a direct correspondence with system pressures. The oscillograph trace provided a time history of the zero-gravity maneuver starting from pullup to pullout. About 4 or 5 seconds of the initial trajectory

following pullup were required to damp out pressure oscillations induced by the pullup maneuver. The portion of the trajectory during which the high-speed camera was operating was also recorded.

Stainless-steel inductance-type pressure transducers, capable of operating in a mercury environment up to $900^{\circ} \mathrm{F}$, were used to measure venturi absolute pressure and venturi pressure drop. Low-temperature transducers were used at all other locations in the system. Each transducer in direct contact with mercury was oriented so that its mounting tube and core axis were on the same horizontal plane as the condensing tube to minimize the head effect. In addition, the mounting tube and core axis were positioned parallel to the lateral axis of the aircraft, which had the least acceleration. Oriented in this way, the $g$ effect of the mercury column in the pressure line was minimal.

Temperatures throughout the system were measured by I. S. A. calibration $\mathrm{K}$ Chromel-Alumel thermocouples. A shielded sheathed thermocouple was immersed in the mercury vapor upstream of the venturi. At other locations, the thermocouple junction was spot welded to the outside surface of the various components such as the heaters. 


\section{Test Procedure}

Prior to each test, the mercury loop was evacuated to approximately 0.060 torr, and the mercury heaters brought to operating temperatures. Mercury flow was initiated and maintained at a low rate for approximately 5 minutes keeping the liquid-vapor interface in the receiver to minimize the presence of noncondensables in the system. The receiver pressure was then increased to the operating value (between 14 and 15 psia), and the gaseous nitrogen flow for condenser tube cooling was regulated to locate the interface at the desired location. In the ground facility, the high-speed pictures were taken at several stations along the condensing length. In this way, condensing characteristics at these stations were recorded for a single setting of flow rate.

In the aircraft, data were taken at both $1-\mathrm{g}$ and zero-gravity conditions. Data at $1 \mathrm{~g}$ (level flight) were determined by the stability of the aircraft in all axes. When the signal from the pilot was given on attaining this condition, the oscillograph recorders and highspeed camera were set in motion. To obtain a complete record of the zero-gravity period, the recording was begun immediately prior to the actual zero-gravity maneuver while still in level flight. The oscillograph recorders and the slow-speed wide-angle-lens camera were started and continued to operate throughout the entire maneuver (the aircraft and zero-gravity maneuver are discussed in ref. 3). During the maneuver, the interface was seen to undergo quite violent movements before stabilizing within the field of view of the high-speed camera. At this point of stabilization, when zero gravity was attained, the high-speed camera was set in motion and operated through the camera reel capacity.

\section{RESULTS AND DISCUSSION}

\section{General Description of Film Observations}

In order to provide an interpretive background for the quantitative study of droplet distribution in a condenser tube, it is helpful first to consider a general qualitative description of the photographic data.

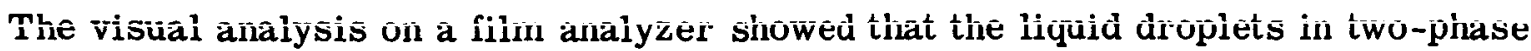
mercury vapor flow in 1-g and zero-gravity environments appeared dispersed throughout the vapor phase approximately in the proportion shown in table I.

The shape of the droplets ranged from spheroids to cigar shaped. The following four shapes were the most frequent on the analyzer screen:

(1) Spherical - small droplets moving in the vapor stream

(2) Spheroid - larger than the most frequent droplet size; moves close to the condenser wall with no oscillation (termed maximum stable droplet) 
TABLE I. - PROPORTION OF DROPLETS DISPERSED IN VAPOR PHASE

\begin{tabular}{|c|c|c|c|c|}
\hline \multirow[t]{2}{*}{ Film } & \multicolumn{2}{|c|}{ Percentage of all droplets } & \multirow{2}{*}{$\begin{array}{l}\text { Assumed } \\
\text { linear vapor } \\
\text { quality, } \\
\mathbf{x}\end{array}$} & \multirow{2}{*}{$\begin{array}{c}\text { Environ- } \\
\text { ment, } \\
\text { g's }\end{array}$} \\
\hline & $\begin{array}{l}\text { Moving on or near } \\
\text { condenser wall }\end{array}$ & $\begin{array}{c}\text { Moving in core of } \\
\text { vapor stream }\end{array}$ & & \\
\hline \multicolumn{5}{|c|}{ Ground test } \\
\hline 1 & 50 & 50 & 0.82 & 1 \\
\hline 2 & 75 & 25 & .36 & 1 \\
\hline 3 & 80 & 20 & .20 & 1 \\
\hline 4 & 85 & 15 & .03 & 1 \\
\hline \multicolumn{5}{|c|}{ Airplane test } \\
\hline 5 & 70 & 30 & 0.03 & 0 \\
\hline 6 & 60 & 40 & .03 & 0 \\
\hline 7 & 50 & 50 & .20 & 0 \\
\hline 8 & 70 & 30 & .03 & 1 \\
\hline 9 & 80 & 20 & .03 & 1 \\
\hline 10 & 70 & 30 & .20 & 1 \\
\hline
\end{tabular}

(3) Ellipsoidal - moves along the bottom on the condenser wall

(4) Irregular - unstable droplets in oscillatory motion on the bottom of the condenser wall close to the vapor-liquid interface

The ratio of the droplet velocity in the vapor core to that of droplet velocity on the condenser wall varied approximately from 5 , close to the inlet, to 2 , near the vaporliquid interface. All the droplets originated on the condenser wall and remained stationery until additional condensation increased the size to the "critical diameter", at which time the vapor drag started to slide them along the wall. The droplet population in a condenser tube increased very rapidly with a decrease in vapor quality. An increase in the droplet population resulted mainly from the breakup process of coalesced droplets.

The effect of gravity on droplet distribution across the condenser tube was pronounced. In zero gravity, droplets were somewhat smaller and more uniformly dispersed across the condenser tube than in a $1-\mathrm{g}$ environment. This effect is discussed in more detail in the following section.

\section{Analysis}

Based on the general qualitative description of droplet dispersion throughout the vapor phase, observed from the films taken of the condensation process, a model was postulated to account quantitatively for a droplet size distribution along the condenser tube. The analysis was carried out in two steps: The first step was to seek the distribution of drop- 
- let frequency, droplet density, and droplet flux along the length of the condenser tube. The second step was to obtain the diameter of a droplet, originated by condensation on the condenser tube wall, at its incipient movement resulting from vapor drag. This diameter is termed the "critical droplet size." In this step, the height of the most frequently occurring droplet on the condenser wall was also obtained.

Basic model. - The basic model of droplet dispersion in the condenser tube is based on the following considerations:

(1) All droplets originated by condensation on the condenser tube wall. The droplets remained on the wall until reaching a critical diameter after which they started to slide toward the interface.

(2) Sliding droplets along the condenser wall increased in size due to coalescence. In most instances, the large droplets were torn apart, and were entrained in the vapor stream as a result of turbulence.

(3) Droplet coalescence and breakup were random in nature.

(4) The droplets were dispersed nonuniformly across the condenser tube. The droplet population on the condenser wall and in the core of the vapor stream varied with vapor quality and gravity conditions.

(5) The droplets were assumed to have a gamma distribution. This assumption is based on a marked similarity between the shape of the histograms of droplet size distribution obtained from measurements and the shape obtained with the gamma probability density function.

(6) As reported by Eckert (ref. 4), in fully developed turbulent flow in a pipe, the laminar-sublayer thickness is well represented by the following relation:

$$
\delta_{b}=63.5 \mathrm{~d}\left(\operatorname{Re}_{\mathrm{v}}\right)^{-0.875}
$$

The laminar-sublayer thickness was obtained from this relation $\delta_{b}$ for ground test conditions, and the results are shown in table $\Pi$. (All symbols are defined in appendix A.)

TABLE II. - ILAMINAR SUBLAYER THICKNESS FOR GROUND TEST CONDITIONS

\begin{tabular}{|c|c|c|c|}
\hline Film & $\begin{array}{c}\text { Vapor quality, } \\
\mathbf{x}\end{array}$ & $\begin{array}{c}\text { Vapor Reynolds } \\
\text { number, } \\
\operatorname{Re}_{\mathbf{v}}^{-0.875}\end{array}$ & $\begin{array}{c}\text { Laminar-sublayer } \\
\text { thickness, } \\
\delta_{\mathrm{b}}\end{array}$ \\
\hline 1 & 0.82 & $13.52 \times 10^{-5}$ & in. \\
2 & .36 & $28.50 \times 10^{-5}$ & 0.004 \\
3 & .20 & $37.60 \times 10^{-5}$ & .008 \\
4 & .03 & $2.27 \times 10^{-3}$ & .010 \\
\hline
\end{tabular}

Therefore, in the range of the condensing length investigated, the ratio of the measured droplet height $\delta_{y}$ of the most frequently occurring size droplet on the condenser wall to the calculated thickness of the laminar sublayer and the ratio of the thickness of the laminar sublayer to the height (taken as $\delta_{c} / 2$ ) of the critical droplet size varied, respectively, as follows: 


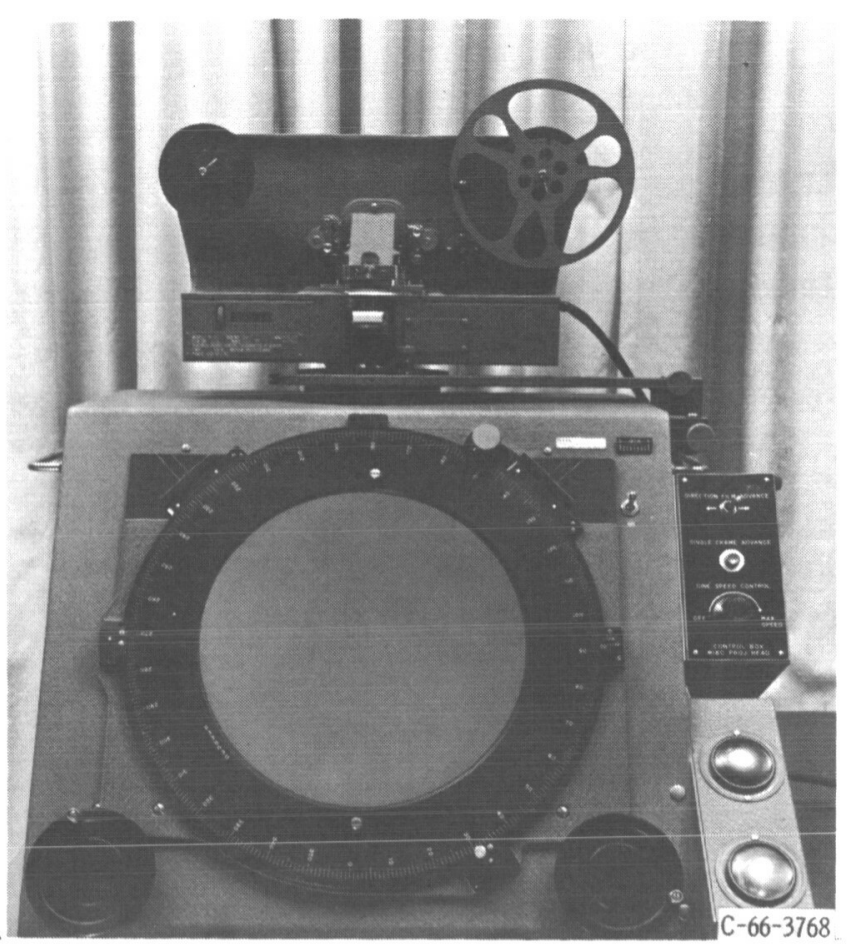

Figure 8. - Motion-picture analyzer.

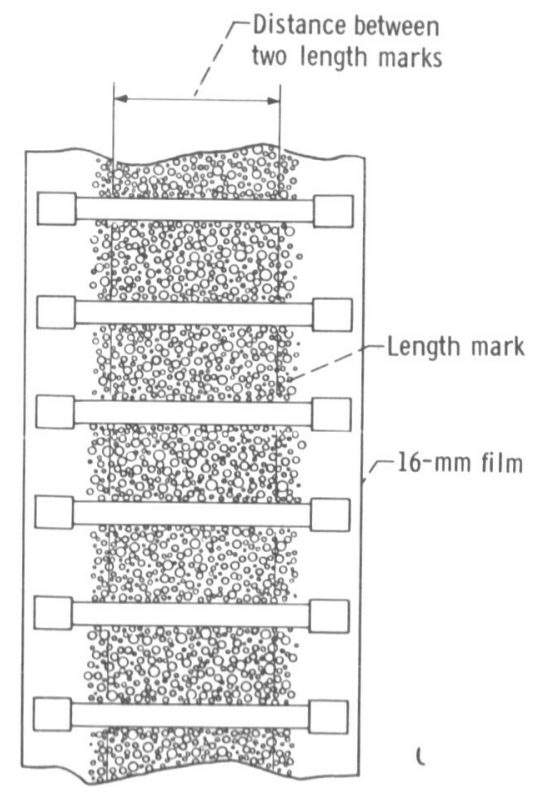

Figure 9. - Schematic representation of dro ; distribution as shown on analyzed screen.

$$
\begin{aligned}
& 2.75 \leq \frac{\delta^{\frac{y}{}}}{\delta_{b}} \leq 0.23 \\
& 1.54 \leq \frac{\delta_{b}}{\delta_{c} / 2} \leq 1.05
\end{aligned}
$$

From these results, the following two assumptions were postulated:

(1) With the exception of the droplets very near the liquid-vapor interface, the height of the most frequent size droplet occurring on a condenser wall would protrude above the laminarsublayer thickness and would be in the region of the turbulent layer.

(2) All the droplets on the condenser wall will be originated by a process of condensation within the laminar-sublayer thickness.

Droplet size distribution. - The droplet sizes and population were developed with the aid of the motion-picture analyzer shown in figure 8. Depending on the lens used, the magnification from film to the analyzer screen was $\times 16.5$ and $\times 27.0$ for the ground tests, and $\times 1.4$ and $\times 2.2$ for the airplane tests. The measurements and counts were made of all droplets that appeared between the two length marks on the tube (as shown in fig. 9) in focus on the film analyzer screen. The measurement of the projected image of a droplet on the analyzer screen was accomplished with crosshair micrometer dials in the $\mathrm{x}$ and $y$-directions. The measurements of droplet sizes and counts of the numbers of each size were made at four specific locations (by two observers) 
ralong the film. For example, for film 1, the measurement of droplet sizes and a count of their number were made at frames 2000, 2200, 2500, and 3000. The frames were counted from the reference point indicated on each film.

The arithmetic mean of the $i^{\text {th }}$ interval of a droplet size analyzed was determined from the following relation

$$
\delta_{z, i}=\frac{\sum_{i=1}^{m} f_{i} \delta_{i}}{\sum_{i=1}^{m} f_{i}}=\frac{\sum_{i=1}^{m} f_{i} \delta_{i}}{N} \quad i=1,2,3, \ldots, m
$$

where

$\delta_{z, i}$ midvalue of $i^{\text {th }}$ interval, termed "group mark"

m number of intervals

$f_{i} \quad$ number of droplets in $i^{\text {th }}$ group interval, called absolute group-mark frequency

$\delta_{\mathbf{i}} \quad$ variable droplet diameter

$\mathrm{N}$ total (average) number of droplets counted between two length marks

The data, thus obtained at various vapor velocities and quality conditions, are listed in tables III(a) to (d) for ground tests (1-g environment) and in tables III(e) to (j) for air plane tests (1-g and zero-gravity environments). In order to compare the droplets of various sizes flowing at various velocities through the section considered on the same time basis, the absolute frequency of each group mark was related to 1 second. Thus,

$$
\mathrm{n}_{\mathrm{w}, \mathrm{i}}=\frac{\mathrm{f}_{\mathrm{i}}}{\Delta \tau_{\mathrm{w}, \mathrm{i}}}
$$

the percentage of droplets on the wall, and

$$
n_{s, i}=\frac{f_{i}}{\Delta \tau_{s, i}}
$$

the percentage of droplets in the stream of the vapor core. The total number of droplets 
TABLE III. - MEASURED,

(a) Film 1 (6 in. from inlet); within interval of frames 2000 to 3000

\begin{tabular}{|c|c|c|c|c|c|c|c|c|c|c|}
\hline \multirow{3}{*}{$\begin{array}{c}\text { Meas- } \\
\text { ured } \\
\text { drop- } \\
\text { let } \\
\text { diam- } \\
\text { eter, } \\
{ }^{\prime} z \\
\text { in. }\end{array}$} & \multicolumn{4}{|c|}{ Frame } & \multirow{3}{*}{$\begin{array}{l}\text { Meas- } \\
\text { ured } \\
\text { fre- } \\
\text { quency, } \\
\mathrm{f}, \\
\text { number } \\
\text { of drop- } \\
\text { lets }\end{array}$} & \multirow{3}{*}{$\begin{array}{l}\text { Average } \\
\text { group } \\
\text { range, } \\
{ }^{\delta} z \\
\text { in. }\end{array}$} & \multirow{3}{*}{$\begin{array}{l}\text { Group } \\
\text { mark, } \\
\delta_{z, i}, \\
\text { in. }\end{array}$} & \multirow{3}{*}{$\begin{array}{c}\text { Abso- } \\
\text { lute } \\
\text { group- } \\
\text { mark } \\
\text { fre- } \\
\text { quency, } \\
\mathrm{f}_{\mathrm{i}} \\
\text { number } \\
\text { of drop- } \\
\text { lets }\end{array}$} & \multirow[b]{3}{*}{$\begin{array}{c}\text { Time for } \\
\text { droplets } \\
\text { of } \mathrm{i}^{\text {th }} \\
\text { group } \\
\text { mark to } \\
\text { travel be- } \\
\text { tween two } \\
\text { length } \\
\text { marks of } \\
\text { condenser } \\
\text { wall, } \\
(\Delta \tau)_{w, i} \\
\text { sec }\end{array}$} & \multirow[b]{3}{*}{$\begin{array}{l}\text { Time for } \\
\text { droplets o } \\
i^{\text {th }} \text { group } \\
\text { mark to } \\
\text { travel be- } \\
\text { tween two } \\
\text { length } \\
\text { marks in } \\
\text { stream of } \\
\text { vapor } \\
\text { core, } \\
(\Delta \tau) \text { s, }{ }^{\prime} \\
\text { sec }\end{array}$} \\
\hline & 2000 & 2200 & 2500 & 3000 & & & & & & \\
\hline & \multicolumn{4}{|c|}{ Number of droplets } & & & & & & \\
\hline 0.005 & 1 & 0 & 2 & 2 & & 0.005 to 0.007 & 0.007 & 4 & 0.01862 & 0.01295 \\
\hline .007 & 4 & 2 & 5 & 0 & & & & & & \\
\hline .008 & 3 & 1 & 6 & 2 & & 0.008 to 0.009 & .009 & 8 & .02620 & .0197 \\
\hline .009 & 5 & 7 & 3 & 4 & $5 J$ & & & & & \\
\hline .010 & 4 & 3 & 5 & 3 & & 0.010 to 0.011 & .011 & 11 & .03110 & .01860 \\
\hline .011 & 7 & 8 & 6 & 8 & $7 \int$ & & & & & \\
\hline .012 & 3 & 0 & 2 & 4 & 27 & 0.012 to 0.013 & 013 & 7 & 01742 & 00824 \\
\hline .013 & 4 & 4 & 6 & 5 & $5\}$ & & & & & \\
\hline .014 & 3 & 4 & 2 & 2 & & 0.014 to 0.015 & .015 & 5 & .01810 & .0082 \\
\hline .015 & 2 & 1 & 3 & 3 & $2 J$ & & & & & \\
\hline .016 & 1 & 0 & 1 & 2 & $1)$ & 0.016 to 0.017 & .017 & 3 & .01470 & .00560 \\
\hline .017 & 2 & 1 & 3 & 2 & $2\}$ & & & & & \\
\hline .018 & 2 & 1 & 0 & 1 & 1 & 0.018 to 0.019 & .019 & 2 & .01380 & .00486 \\
\hline .019 & 0 & 2 & 1 & 1 & 1 & & & & & \\
\hline .020 & 1 & 0 & 1 & 0 & 01 & 0.020 to 0.021 & .021 & 1 & .01118 & .00435 \\
\hline .021 & 1 & 2 & 0 & 1 & $1 \int$ & & .021 & & & \\
\hline Total & & & & & & & & 41 & & \\
\hline
\end{tabular}


DROPLET DISTRIBUTION

counted from reference point; ground test; 1 -g environment

\begin{tabular}{|c|c|c|c|c|c|c|c|}
\hline $\begin{array}{c}\begin{array}{c}\mathbf{f}_{\mathbf{i}} \delta_{z, i}, \\
\text { droplets }\end{array} \\
\frac{\sec }{}\end{array}$ & $\begin{array}{l}\text { Percent- } \\
\text { age of } \\
\text { droplets } \\
\text { on wall, } \\
\mathrm{n}_{w, i} \\
\frac{\text { droplets }}{\text { sec }}\end{array}$ & $\begin{array}{l}\text { Percent- } \\
\text { age of } \\
\text { droplets } \\
\text { in vapor } \\
\text { core, } \\
n_{\mathbf{s}, i}, \\
\frac{\text { droplets }}{\text { sec }}\end{array}$ & $\begin{array}{c}\text { Total droplets } \\
\text { passing through } \\
\text { reference point } \\
\text { along con- } \\
\text { denser tube, } \\
\mathrm{n}_{\mathrm{t}, \mathrm{i}}=\mathrm{n}_{\mathrm{w}, \mathrm{i}}+\mathrm{n}_{\mathrm{s}, \mathrm{i}}, \\
\frac{\text { droplets }}{\text { sec }}\end{array}$ & $\begin{array}{c}\text { Average } \\
\text { number } \\
\text { of drop- } \\
\text { lets per } \\
\text { unit } \\
\text { volume } \\
\text { of con- } \\
\text { denser } \\
\text { tube, } \\
n_{v, i}, \\
\frac{\text { droplets }}{\text { in. }}\end{array}$ & 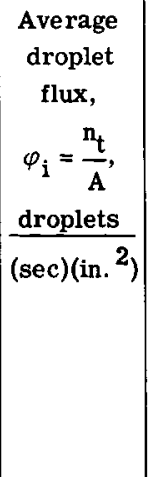 & $\mathrm{n}_{\mathrm{t}, \mathrm{i}^{\mathrm{i}} \mathrm{z}, \mathrm{i}}$ & $\begin{array}{l}\text { Total } \\
\text { area } \\
\text { of } \\
\text { histo- } \\
\text { gram, } \\
\text { A }_{t, 1}\end{array}$ \\
\hline 0.028 & 107 & 154 & 261 & 35 & 1730 & 1.83 & 0.0875 \\
\hline .072 & 153 & 203 & 356 & 70 & 2340 & 3.21 & .1201 \\
\hline .121 & 177 & 296 & 473 & 96 & 3110 & 5.21 & .1580 \\
\hline .091 & 201 & 424 & 625 & 61 & 4110 & 8.13 & .2090 \\
\hline .075 & 138 & 305 & 443 & 44 & 3910 & 6.64 & .1480 \\
\hline .051 & 102 & 268 & 370 & 26 & 2430 & 6.28 & .1240 \\
\hline .038 & 72 & 206 & 278 & 17 & 1828 & 5.28 & .0927 \\
\hline .021 & 45 & 115 & 160 & 9 & 1051 & 3.36 & .0534 \\
\hline 0.497 & & & 2966 & 358 & & 39.94 & 0.992 \\
\hline
\end{tabular}


TABLE III. - Continued. MEASURED.

(b) Film 2 (42 in. from inlet); within interval of frames 1000 to 2500

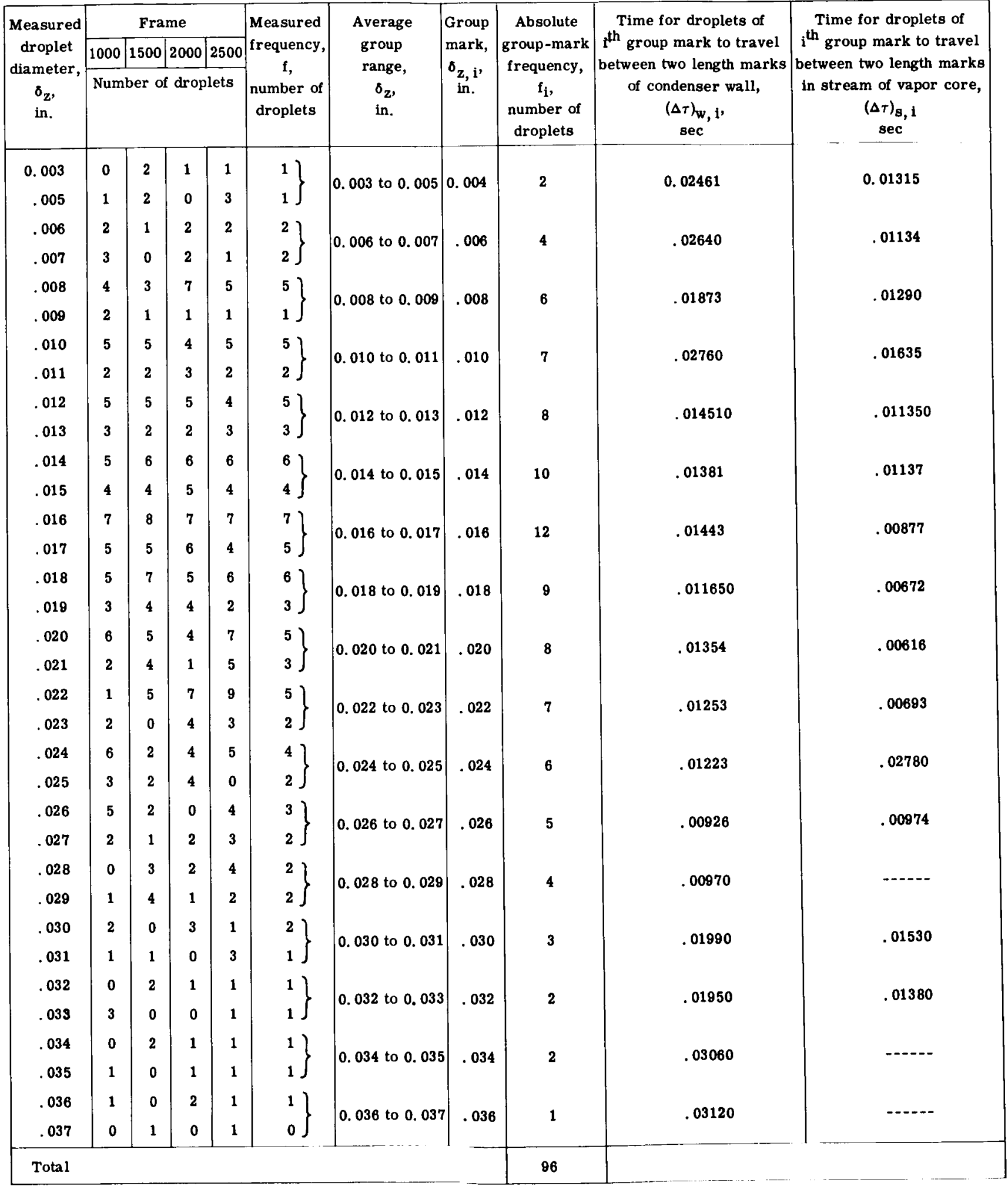




\section{DROPLET DISTRIBUTION}

counted from reference point; ground test; 1 -g environment

\begin{tabular}{|c|c|c|c|c|c|c|c|}
\hline $\begin{array}{c}\mathbf{f}_{\mathfrak{i} \delta_{z, \mathfrak{i}},} \\
\text { droplets }\end{array}$ & $\begin{array}{c}\text { Percentage of } \\
\text { droplets on } \\
\text { wall, } \\
\mathbf{n}_{\mathrm{w}, \mathrm{i}}, \\
\frac{\text { droplets }}{\text { sec }}\end{array}$ & $\begin{array}{l}\text { Percentage of } \\
\text { droplets in } \\
\text { vapor core, } \\
\text { n }_{\mathbf{s}, \mathbf{i}} \\
\frac{\text { droplets }}{\text { sec }}\end{array}$ & $\begin{array}{l}\text { Total droplets passing } \\
\text { through reference point } \\
\text { along condenser tube, } \\
n_{t, 1}=n_{w, i}+n_{s, i^{\prime}} \\
\frac{\text { droplets }}{\text { sec }}\end{array}$ & $\begin{array}{c}\text { Average number of } \\
\text { droplets per unit vol- } \\
\text { ume of condenser tube, } \\
\mathrm{n}_{\mathrm{v}, \mathrm{i}} \text {, } \\
\frac{\text { droplets }}{\text { in. }}\end{array}$ & $\begin{array}{c}\text { Average drop- } \\
\text { let flux, } \\
\varphi_{i}=\frac{n_{t}}{A} \\
\frac{\text { droplets }}{(\sec )\left(\text { in. }^{2}\right)}\end{array}$ & $n_{t, i} \delta_{z, i}$ & $\begin{array}{c}\text { Total area of } \\
\text { histogram, } \\
A_{t, i}\end{array}$ \\
\hline 0.008 & 61 & 38 & 99 & 13 & 651 & 0.040 & 0.01368 \\
\hline .024 & 113 & 86 & 201 & 26 & 1322 & 1.20 & .02778 \\
\hline .048 & 240 & 116 & 356 & 39 & 2342 & 2.85 & .04920 \\
\hline .070 & 190 & 107 & 297 & 46 & 1954 & 2.97 & .04105 \\
\hline .096 & 413 & 176 & 589 & 53 & 3875 & 7.06 & .08140 \\
\hline .140 & 543 & 220 & 763 & 66 & 5020 & 10.76 & .10545 \\
\hline .192 & 622 & 342 & 964 & 79 & 6342 & 15.40 & .13322 \\
\hline .162 & 580 & 337 & 917 & 59 & 6033 & 16.50 & .12673 \\
\hline .160 & 442 & 325 & 767 & 53 & 5046 & 15.38 & .10600 \\
\hline .154 & 418 & 252 & 670 & 46 & 4408 & 14.75 & .09259 \\
\hline .144 & 367 & 53 & 420 & 39 & 2763 & 10.10 & .05804 \\
\hline .130 & 404 & 128 & 532 & 33 & 3500 & 13.80 & .07352 \\
\hline .112 & 310 & 0 & 310 & 26 & 2040 & 8.66 & .04284 \\
\hline . ชิงิ์ & $\mathbf{i} \mathbf{i z}$ & $4 \hat{8}$ & î̂ô & 20 & 1053 & $\frac{1}{4} .80$ & .02211 \\
\hline .064 & 77 & 36 & 113 & 13 & 743 & 3.62 & .01562 \\
\hline .068 & 49 & 0 & 49 & 13 & 322 & 1.67 & .00677 \\
\hline .036 & 25 & 0 & 25 & 7 & 164 & .90 & .00346 \\
\hline 1.698 & & & 7232 & 631 & & 130.82 & 0.99476 \\
\hline
\end{tabular}


TABLE III. - Continued. MEASURED

(c) Film 3 (54.6 in. from inlet); within interval of frames 1200 to 2000

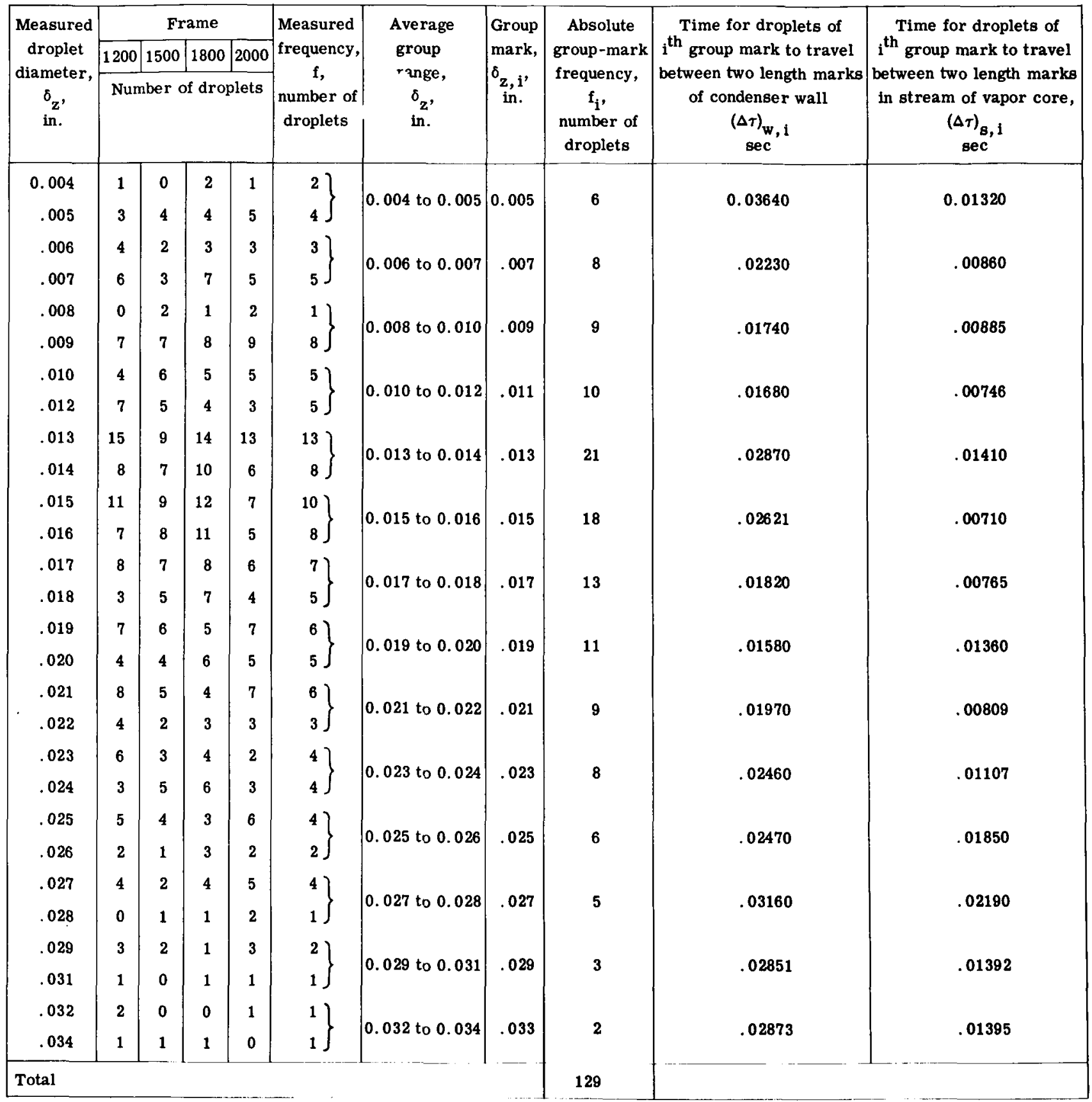


DROPLET DISTRIBUTION

counted from reference point; ground test; 1 -g environment

\begin{tabular}{|c|c|c|c|c|c|c|c|}
\hline 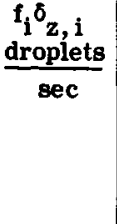 & $\begin{array}{l}\text { Percentage of } \\
\text { droplets on } \\
\text { wall, } \\
\text { nw, i' } \\
\frac{\text { droplets }}{\text { sec }}\end{array}$ & $\begin{array}{l}\text { Percentage of } \\
\text { droplets in } \\
\text { vapor core, } \\
\text { ns,i' }_{\text {droplets }} \\
\frac{\text { sec }}{}\end{array}$ & $\begin{array}{l}\text { Total droplets passing } \\
\text { through reference point } \\
\text { along condenser tube, } \\
\qquad \begin{aligned} & \mathbf{n}_{\mathbf{t}, \mathbf{i}}=\mathbf{n}_{\mathbf{w}, \mathbf{i}}+\mathbf{n}_{\mathbf{s}, \mathbf{i}} \\
& \frac{\text { droplets }}{\text { sec }}\end{aligned}\end{array}$ & $\begin{array}{l}\text { Average number of } \\
\text { droplets per unit vol- } \\
\text { ume of condenser tube, } \\
\frac{n_{v, i},}{\text { in. } 3}\end{array}$ & $\begin{array}{l}\text { Average drop- } \\
\text { let flux, } \\
\varphi_{\mathbf{i}}=\frac{\mathbf{n}_{\mathbf{t}}}{\mathbf{A}} \\
\frac{\text { droplets }}{(\mathrm{sec})\left(\text { in. }^{2}\right)}\end{array}$ & $n_{t, i}{ }^{\delta} z, i$ & $\begin{array}{c}\text { Total area of } \\
\text { histogram, } \\
\mathbf{A}_{\mathrm{t}, \mathrm{i}}\end{array}$ \\
\hline 0.030 & 132 & 91 & 223 & 39 & 1467 & 1.1150 & 0.0308 \\
\hline .056 & 287 & 186 & 473 & 53 & 3112 & 3.3110 & .0653 \\
\hline .081 &. .414 & 201 & 615 & 59 & 4047 & 5.5350 & .0849 \\
\hline .110 & 482 & 268 & 750 & 66 & 4935 & 8. 2500 & .1035 \\
\hline .273 & 585 & 300 & 885 & 138 & 5823 & 11.5050 & .1221 \\
\hline .270 & 550 & 507 & 1057 & 118 & 6955 & 15.8550 & .1459 \\
\hline .221 & 570 & 341 & 911 & 85 & 5994 & 15.4870 & .1257 \\
\hline .209 & 556 & 162 & 718 & 72 & 4724 & 13.6420 & .0991 \\
\hline .189 & 364 & 223 & 587 & 59 & 3862 & 12.3270 & .0810 \\
\hline .184 & 261 & 145 & 406 & 53 & 2671 & 9.3380 & .0560 \\
\hline .150 & 194 & 65 & 259 & 39 & 1704 & 6.4750 & .0357 \\
\hline .135 & 126 & 46 & 172 & 33 & 1132 & 4.6440 & .0237 \\
\hline .087 & 63 & 43 & 106 & 20 & 698 & 3.0740 & .0146 \\
\hline .066 & 56 & 3 & 85 & 13 & 559 & 2. 8050 & .0059 \\
\hline 2. 063 & & & 7247 & 847 & & 113.363 & 0.9941 \\
\hline
\end{tabular}


TABLE III. - Continued, MEASURED

(d) Film 4 (67. 8 in. from inlet); within interval of frames 1000 to

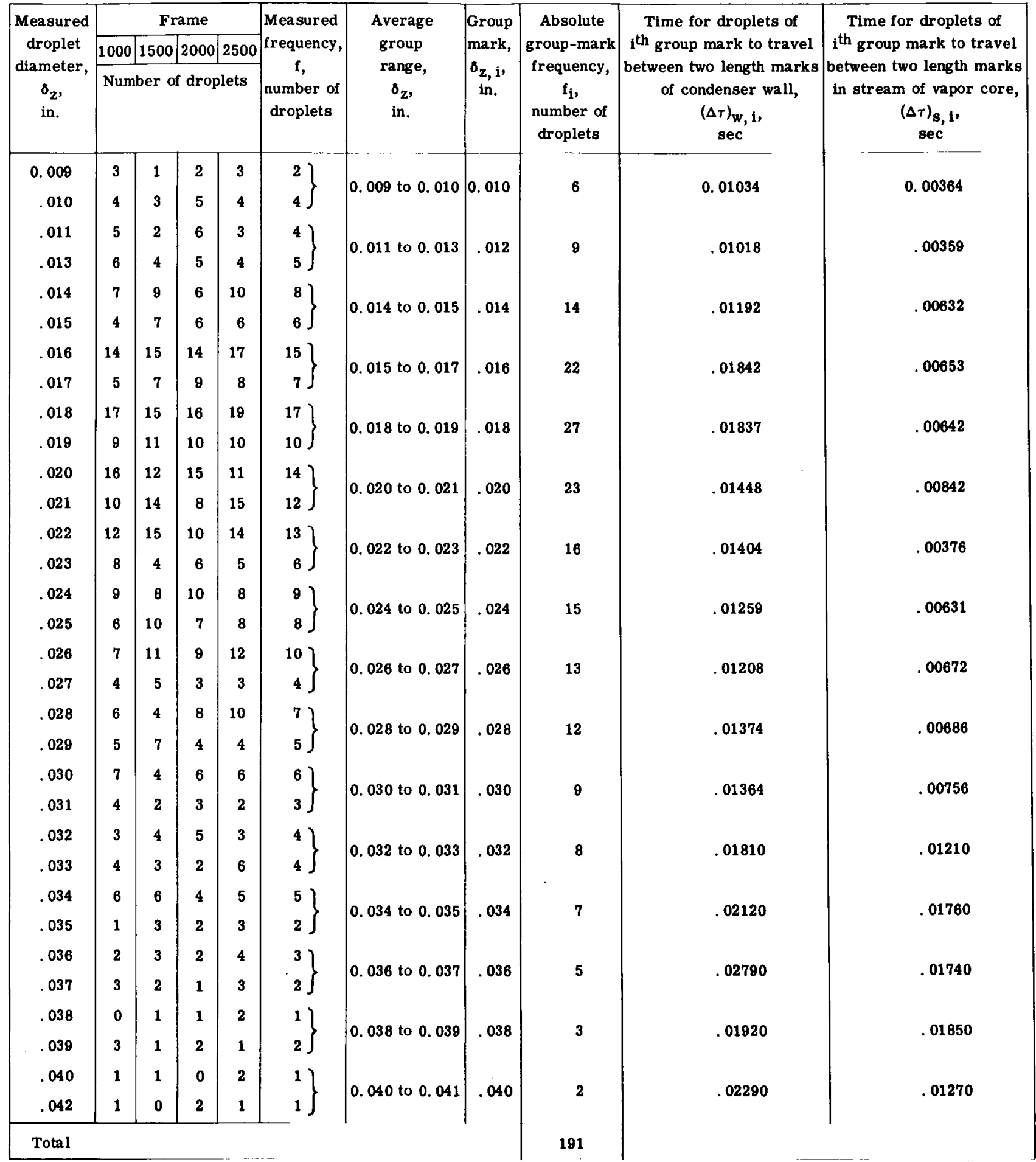




\section{- DROPLET DISTRIBUTION}

2500 counted from reference point; ground test; 1 -g environment

\begin{tabular}{|c|c|c|c|c|c|c|c|}
\hline$\frac{\begin{array}{c}\mathbf{f}_{\mathbf{i}} \boldsymbol{\delta}_{\mathbf{z}, \mathbf{i}} \\
\text { droplets }\end{array}}{\text { sec }}$ & $\begin{array}{c}\text { Percentage of } \\
\text { droplets on } \\
\text { wall, } \\
\mathrm{n}_{\mathbf{w}, \mathbf{i}} \\
\text { droplets } \\
\text { sec }\end{array}$ & $\begin{array}{c}\text { Percentage of } \\
\text { droplets in } \\
\text { vapor core, } \\
\mathrm{n}_{\mathbf{s}, \mathbf{i}} \\
\frac{\text { droplets }}{\text { sec }}\end{array}$ & $\begin{array}{c}\text { Total droplets passing } \\
\text { through reference point } \\
\text { along condenser tube, } \\
n_{t, i}=n_{w, i}+n_{s, i} \\
\frac{\text { droplets }}{\text { sec }}\end{array}$ & $\begin{array}{c}\text { Average number of } \\
\text { droplets per unit vol- } \\
\text { ume of condenser tube, } \\
\mathrm{n}_{\mathrm{v}, \mathrm{i}} \text {, } \\
\frac{\text { droplets }}{\text { in. }^{3}}\end{array}$ & $\begin{array}{c}\text { Average drop- } \\
\text { let flux, } \\
\varphi_{i}=\frac{\mathrm{n}_{\mathrm{t}}}{\mathrm{A}} \\
\text { droplets } \\
\left.\text { (sec)(in. }{ }^{2}\right)\end{array}$ & $n_{t, i^{\delta}} z, i$ & $\begin{array}{c}\text { Total area of } \\
\text { histogram, } \\
\mathbf{A}_{\mathrm{t}, \mathrm{i}}\end{array}$ \\
\hline 0.060 & 493 & 247 & 740 & 39 & 4880 & 7.40 & 0.04887 \\
\hline .108 & 747 & 373 & 1120 & 59 & 7360 & 13.42 & .07399 \\
\hline .196 & 998 & 332 & 1330 & 92 & 8740 & 18.61 & .08786 \\
\hline .352 & 1014 & 506 & 1520 & 145 & 9980 & 24.30 & .10042 \\
\hline .486 & 1125 & 355 & 1480 & 178 & 11750 & 26.70 & .09777 \\
\hline .460 & 1350 & 409 & 1752 & 151 & 11570 & 35.20 & .11620 \\
\hline .352 & 967 & 637 & 1604 & 105 & 10560 & 35.30 & .10597 \\
\hline .360 & 1012 & 357 & 1369 & 99 & 6980 & 33.60 & .09044 \\
\hline .338 & 914 & 290 & 1204 & 86 & 7930 & 31.30 & .07954 \\
\hline .336 & 744 & 262 & 1006 & 79 & 6610 & 28.20 & .06646 \\
\hline .270 & 562 & 178 & 740 & 59 & 4860 & 22.20 & .04883 \\
\hline .256 & 375 & 99 & 474 & 53 & 3120 & 15.20 & .03131 \\
\hline .238 & 281 & 60 & 341 & 46 & 2240 & 11.60 & .02253 \\
\hline .180 & 152 & 43 & 195 & 33 & 1282 & 7.02 & .01288 \\
\hline .114 & 113 & 24 & 157 & 20 & 1032 & 5.97 & .01037 \\
\hline .080 & 74 & 24 & 98 & 13 & 644 & 3.93 & .00647 \\
\hline 4.186 & & & 15137 & 1257 & & 328.55 & 0.9995 \\
\hline
\end{tabular}


TABLE III. - Continued. MEASURED .

(e) Film 8 (47 in. from inlet); within intervals of frames 3200 to 3800 counted from reference point; airplane mean vapor velocity, 43.50 feet per second.

\begin{tabular}{|c|c|c|c|c|c|c|c|c|c|}
\hline \multirow{3}{*}{$\begin{array}{c}\text { Measured } \\
\text { droplet } \\
\text { diameter, } \\
\delta_{z} \\
\text { in. }\end{array}$} & \multicolumn{4}{|c|}{ Frame } & \multirow{3}{*}{$\begin{array}{c}\text { Measured } \\
\text { frequency, } \\
\text { f, } \\
\text { number of } \\
\text { droplets }\end{array}$} & \multirow{3}{*}{$\begin{array}{c}\text { Average } \\
\text { group } \\
\text { range, } \\
\delta_{z} \\
\text { in. }\end{array}$} & \multirow{3}{*}{$\begin{array}{l}\text { Group } \\
\text { mark, } \\
{ }^{\delta} \mathrm{z}, \mathrm{i} \\
\text { in. }\end{array}$} & \multirow{3}{*}{$\begin{array}{c}\text { Absolute group- } \\
\text { mark frequency, } \\
\mathbf{f}_{\mathbf{i}}, \\
\text { number of } \\
\text { droplets }\end{array}$} & \multirow{3}{*}{$\begin{array}{l}\text { Time for drop- } \\
\text { lets of } \mathbf{i}^{\text {th }} \text { group } \\
\text { mark to travel } \\
\text { between two } \\
\text { length marks of } \\
\text { condenser wall, } \\
(\Delta \tau)_{w, i} \\
\text { sec }\end{array}$} \\
\hline & 3200 & 3400 & 3600 & 3800 & & & & & \\
\hline & \multicolumn{4}{|c|}{ Number of droplets } & & & & & \\
\hline $\begin{array}{r}0.013 \\
.014\end{array}$ & $\begin{array}{l}1 \\
1\end{array}$ & $\begin{array}{l}0 \\
2\end{array}$ & $\begin{array}{l}0 \\
1\end{array}$ & $\begin{array}{l}1 \\
1\end{array}$ & $\left.\begin{array}{l}0 \\
1\end{array}\right\}$ & 0.013 to 0.014 & 0.014 & 1 & $-\lambda-1$ \\
\hline .015 & 1 & 0 & 0 & 1 & 0 & 0.015 to 0.016 & .016 & 1 & 0.02000 \\
\hline .016 & 3 & 1 & 0 & 1 & 1 & & & & \\
\hline $\begin{array}{l}.017 \\
.018\end{array}$ & $\begin{array}{l}0 \\
4\end{array}$ & $\begin{array}{l}1 \\
0\end{array}$ & $\begin{array}{l}3 \\
3\end{array}$ & $\begin{array}{l}1 \\
2\end{array}$ & $\left.\begin{array}{l}1 \\
2\end{array}\right\}$ & 0.017 to 0.018 & .018 & 3 & .02083 \\
\hline $\begin{array}{l}.019 \\
.020\end{array}$ & $\begin{array}{l}0 \\
2\end{array}$ & $\begin{array}{l}3 \\
4\end{array}$ & $\begin{array}{l}1 \\
2\end{array}$ & $\begin{array}{l}1 \\
3\end{array}$ & $\begin{array}{l}1 \\
3\end{array}$ & 0.019 to 0.020 & .020 & 4 & .01156 \\
\hline $\begin{array}{r}.021 \\
.022\end{array}$ & $\begin{array}{l}4 \\
2\end{array}$ & $\begin{array}{l}1 \\
0\end{array}$ & $\begin{array}{l}5 \\
4\end{array}$ & $\begin{array}{l}2 \\
2\end{array}$ & $\begin{array}{l}3 \\
2\end{array}$ & 0.021 to 0.022 & .022 & 5 & .02033 \\
\hline $\begin{array}{l}.023 \\
.024\end{array}$ & $\begin{array}{l}1 \\
6\end{array}$ & $\begin{array}{l}2 \\
5\end{array}$ & $\begin{array}{l}4 \\
2\end{array}$ & $\begin{array}{l}0 \\
4\end{array}$ & $\begin{array}{l}2 \\
4\end{array}$ & 0.023 to 0.024 & .024 & 6 & .01408 \\
\hline $\begin{array}{l}.025 \\
.026\end{array}$ & $\begin{array}{l}3 \\
9\end{array}$ & $\begin{array}{l}1 \\
7\end{array}$ & $\begin{array}{l}7 \\
8\end{array}$ & $\begin{array}{l}4 \\
5\end{array}$ & $\begin{array}{l}4 \\
7\end{array}$ & 0.025 to 0.026 & .026 & 11 & .02273 \\
\hline $\begin{array}{r}.027 \\
.028\end{array}$ & $\begin{array}{l}0 \\
7\end{array}$ & $\begin{array}{l}2 \\
3\end{array}$ & $\begin{array}{l}3 \\
4\end{array}$ & $\begin{array}{l}2 \\
3\end{array}$ & $\left.\begin{array}{l}2 \\
4\end{array}\right\}$ & 0.027 to 0.028 & .028 & 6 & .01840 \\
\hline $\begin{array}{l}.029 \\
.030\end{array}$ & $\begin{array}{l}0 \\
3\end{array}$ & $\begin{array}{l}2 \\
1\end{array}$ & $\begin{array}{l}1 \\
3\end{array}$ & $\begin{array}{l}1 \\
2\end{array}$ & $\begin{array}{l}1 \\
2\end{array}$ & 0.029 to 0.030 & .030 & 3 & .02113 \\
\hline $\begin{array}{l}.031 \\
.032\end{array}$ & $\begin{array}{l}4 \\
3\end{array}$ & $\begin{array}{l}0 \\
1\end{array}$ & $\begin{array}{l}3 \\
2\end{array}$ & $\begin{array}{l}2 \\
3\end{array}$ & $\begin{array}{l}2 \\
2\end{array}$ & 0.031 to 0.032 & .032 & 4 & .01460 \\
\hline $\begin{array}{r}.033 \\
.034\end{array}$ & $\begin{array}{l}3 \\
2\end{array}$ & $\begin{array}{l}0 \\
4\end{array}$ & $\begin{array}{l}1 \\
0\end{array}$ & $\begin{array}{l}1 \\
3\end{array}$ & $\left.\begin{array}{l}1 \\
1\end{array}\right)$ & 0.033 to 0.034 & .034 & 2 & .01266 \\
\hline $\begin{array}{l}.035 \\
.036\end{array}$ & $\begin{array}{l}1 \\
1\end{array}$ & $\begin{array}{l}1 \\
3\end{array}$ & $\begin{array}{l}2 \\
4\end{array}$ & $\begin{array}{l}0 \\
0\end{array}$ & $\begin{array}{l}1 \\
2\end{array}$ & 0.035 to 0.036 & .036 & 3 & .01829 \\
\hline $\begin{array}{l}.037 \\
.038\end{array}$ & $\begin{array}{l}1 \\
0\end{array}$ & $\begin{array}{l}0 \\
1\end{array}$ & $\begin{array}{l}1 \\
4\end{array}$ & $\begin{array}{l}3 \\
4\end{array}$ & $\left.\begin{array}{l}1 \\
2\end{array}\right\}$ & 0.037 to 0.038 & .038 & 3 & .02027 \\
\hline $\begin{array}{l}.039 \\
.040\end{array}$ & $\begin{array}{l}3 \\
2\end{array}$ & $\begin{array}{l}1 \\
4\end{array}$ & $\begin{array}{l}1 \\
0\end{array}$ & $\begin{array}{l}2 \\
3\end{array}$ & $\left.\begin{array}{l}2 \\
2\end{array}\right\}$ & 0.039 to 0.040 & .040 & 4 & .02299 \\
\hline Total & & & & & & & & 56 & \\
\hline
\end{tabular}


DROPLET DISTRIBUTION

test, zero-gravity environment; vapor quality, 0.20 ; total weight flow rate, 0.0329 pound per second; local

\begin{tabular}{|c|c|c|c|c|c|c|}
\hline $\begin{array}{l}\text { Time for droplets } \\
\text { of ith group mark } \\
\text { to travel between } \\
\text { two length marks } \\
\text { in stream of } \\
\text { vapor core, } \\
(\Delta \tau)_{8, i} \\
\text { sec }\end{array}$ & $\frac{\begin{array}{c}f_{i}{ }^{\delta} z, i \\
\text { droplets }\end{array}}{\sec }$ & $\begin{array}{l}\text { Total droplets } \\
\text { passing through } \\
\text { reference point } \\
\text { along con- } \\
\text { denser tube, } \\
\mathrm{n}_{\mathrm{t}, \mathrm{i}}=\mathrm{n}_{\mathrm{w}, \mathrm{i}}+\mathrm{n}_{\mathrm{s}, \mathrm{i}} \text { ' } \\
\frac{\text { droplets }}{\text { sec }}\end{array}$ & $\begin{array}{c}\text { Average number } \\
\text { of droplets per } \\
\text { unit volume of } \\
\text { condenser tube, } \\
n_{v, i}, \\
\frac{\text { droplets }}{\text { in. }^{3}}\end{array}$ & $\begin{array}{c}\begin{array}{c}\text { Average } \\
\text { drop- } \\
\text { let flux, } \\
\varphi_{\mathrm{i}}=\frac{\mathrm{n}_{\mathrm{t}}}{\mathrm{A}} \\
\text { droplets } \\
(\sec )\left(\mathrm{in}^{2}\right)^{2}\end{array}\end{array}$ & $n_{t, i}{ }^{\delta} z, i$ & $\begin{array}{c}\text { Total area } \\
\text { of histogram, } \\
\mathbf{A}_{\mathrm{t}, \mathrm{i}}\end{array}$ \\
\hline 0.00562 & 0.014 & 89 & 8 & 709 & 1.25 & 0.01465 \\
\hline .00400 & .016 & 150 & 8 & 1194 & 2.40 & .02470 \\
\hline .00595 & .054 & 324 & 24 & 2580 & 5.83 & .05334 \\
\hline .00727 & .080 & 448 & 32 & 3567 & 8.96 & .07376 \\
\hline .00577 & .110 & 556 & 40 & 4427 & 12.23 & .09154 \\
\hline .00580 & .144 & 730 & 48 & 5812 & 17.52 & .12018 \\
\hline .00794 & .286 & 935 & 88 & 7444 & 24.31 & .15393 \\
\hline .00585 & .168 & 676 & 48 & 5382 & 18.93 & .11129 \\
\hline .00847 & .090 & 248 & 24 & 1975 & 7.44 & .04083 \\
\hline .00454 & .128 & 578 & 32 & 4602 & 18.50 & .09516 \\
\hline .00455 & .068 & 299 & 16 & 2381 & 10.17 & .04923 \\
\hline .00493 & .108 & 386 & 24 & 3073 & 13.90 & .06355 \\
\hline .00647 & .114 & 306 & 24 & 2436 & 11.63 & .05038 \\
\hline .00763 & .160 & 349 & 32 & 2779 & 13.96 & .05746 \\
\hline & & 6074 & 448 & & 167.03 & 1.00000 \\
\hline
\end{tabular}


TABLE III. - Continued. MEASURED

(f) Film 5 (60 in. from inlet); within interval of frames 3000 to 3600 counted from reference point; airplane test; vapor velocity, 7.17 feet per second.

\begin{tabular}{|c|c|c|c|c|c|c|c|c|c|}
\hline \multirow{3}{*}{$\begin{array}{c}\text { Measured } \\
\text { droplet } \\
\text { diameter, } \\
\delta_{z} \\
\text { in. }\end{array}$} & \multicolumn{4}{|c|}{ Frame } & \multirow{3}{*}{$\begin{array}{l}\text { Measured } \\
\text { frequency, } \\
\mathbf{f}, \\
\text { number of } \\
\text { droplets }\end{array}$} & \multirow{3}{*}{$\begin{array}{l}\text { Average } \\
\text { group } \\
\text { range, } \\
\delta_{z}, \\
\text { in. }\end{array}$} & \multirow{3}{*}{$\begin{array}{l}\text { Group } \\
\text { mark, } \\
\delta_{z, i} \\
\text { in. }\end{array}$} & \multirow{3}{*}{$\begin{array}{c}\text { Absolute group- } \\
\text { mark frequency, } \\
\mathbf{f}_{\mathbf{i}} \text { ' } \\
\text { number of } \\
\text { droplets }\end{array}$} & \multirow{3}{*}{$\begin{array}{l}\text { Time for drop- } \\
\text { lets of ith group } \\
\text { mark to travel } \\
\text { between two } \\
\text { length marks of } \\
\text { condenser wall, } \\
(\Delta \tau)_{w, 1} \\
\text { sec }\end{array}$} \\
\hline & 3000 & 3200 & 3400 & 3600 & & & & & \\
\hline & \multicolumn{4}{|c|}{ Number of droplets } & & & & & \\
\hline 0.011 & 1 & 0 & 1 & 0 & 0 & 0011 to 0012 & 0012 & 1 & 0.01458 \\
\hline .012 & 2 & 1 & 0 & 1 & 1 & 0.02100 .016 & 0.012 & 2 & 0.01800 \\
\hline .013 & 1 & 0 & 0 & 1 & 0 & & & & \\
\hline .014 & 1 & 1 & 2 & 1 & 1 & 0.013 to 0.014 & .014 & 1 & .00875 \\
\hline .015 & $\mathbf{0}$ & 1 & 3 & 1 & 1 & 0015 to 0018 & 010 & & \\
\hline .016 & 1 & 0 & 2 & 1 & 1 & 0.01500 .010 & .010 & $\mathbf{2}$ & .00972 \\
\hline .017 & 2 & 3 & 4 & 0 & 2 & 0017 to 0018 & 018 & 3 & 00004 \\
\hline .018 & 0 & 1 & 2 & 2 & 1 & 0.02100 .010 & .010 & $\boldsymbol{J}$ & .00094 \\
\hline .019 & 2 & 3 & 1 & 2 & 2 & & & & \\
\hline .020 & 3 & 2 & 2 & 2 & 2 & 0.019 to 0.020 & .020 & 4 & .01094 \\
\hline .021 & 4 & 1 & 5 & 2 & 3 & 0.021 to 0.022 & 022 & 7 & .00948 \\
\hline .022 & 6 & 3 & 2 & 5 & 4 & 0.001 to 0.024 & $.02 \mathbf{a}$ & r & .00840 \\
\hline .023 & 3 & 2 & 4 & 2 & 3 & & & & \\
\hline .024 & 4 & 6 & 1 & 2 & 3 & 0.023 to 0.024 & .024 & 6 & .00719 \\
\hline .025 & 5 & 3 & 7 & 4 & 5 & 0005100000 & & & \\
\hline .026 & 4 & 3 & 2 & 2 & 3 & 0.025 to 0.026 & .026 & 8 & .00523 \\
\hline .027 & 7 & 6 & 3 & 3 & 5 & & & & \\
\hline .028 & 6 & 8 & 5 & 6 & 6 & 0.027 to 0.028 & .028 & 11 & .01878 \\
\hline .029 & 3 & 7 & 2 & 5 & 4 & 0.028 to 0.030 & 030 & 10 & 03804 \\
\hline .030 & 8 & 3 & 5 & 7 & 6 & & & & \\
\hline .031 & 3 & 2 & 3 & 5 & 3 & 0021 to 0032 & & 8 & \\
\hline .032 & 5 & 4 & 7 & 3 & 5 & 0.031 to 0.032 & .032 & 8 & .01223 \\
\hline .033 & 2 & 5 & 6 & 4 & 4 & & & & \\
\hline .034 & 1 & 0 & 5 & 6 & 3 & 0.033 to 0.034 & .034 & 7 & .02112 \\
\hline .035 & 6 & 1 & 0 & 0 & 2 & 0025 to 0038 & 030 & $B$ & 09498 \\
\hline .036 & 8 & 2 & 1 & 6 & 4 & 0.00000 .000 & . 030 & 0 & .02428 \\
\hline .037 & 0 & 1 & 6 & 5 & 3 & & & & \\
\hline .038 & 1 & 3 & 2 & 1 & 2 & 0.037 to 0.038 & .038 & 5 & .01311 \\
\hline .038 & 2 & 0 & 1 & 0 & 1 & & & & \\
\hline .040 & 3 & 4 & 0 & 2 & $2\}$ & 0.039 to 0.040 & .040 & 3 & .02727 \\
\hline .041 & 5 & 0 & 6 & 1 & 3 & 0041 to 0042 & & & \\
\hline .042 & 1 & 2 & 0 & 1 & $1 \int$ & 0.041 to 0.042 & .042 & 4 & .02800 \\
\hline Total & & & & & & & & 86 & \\
\hline
\end{tabular}


DROPLET DISTRIBUTION

zero-gravity environment; vapor quality, 0.03 ; total weight flow rate, 0.0435 pound per second; local mean

\begin{tabular}{|c|c|c|c|c|c|c|}
\hline $\begin{array}{l}\text { Time for droplets } \\
\text { of } i^{\text {th }} \text { group mark } \\
\text { to travel between } \\
\text { two length marks } \\
\text { in stream of } \\
\text { vapor core, } \\
(\Delta \tau)_{8, i} \\
\text { sec }\end{array}$ & $\begin{array}{c}\mathbf{f}_{\mathbf{i}^{8} \mathrm{z}, \mathrm{i}} \\
\frac{\text { droplets }}{\mathrm{sec}}\end{array}$ & $\begin{array}{l}\text { Total droplets } \\
\text { passing through } \\
\text { reference point } \\
\text { along con- } \\
\text { denser tube, } \\
\mathbf{n}_{\mathrm{t}, \mathrm{i}}=\mathrm{n}_{\mathrm{w}, \mathrm{i}}+\mathrm{n}_{\mathrm{s}, \mathrm{i}} \\
\frac{\text { droplets }}{\text { sec }}\end{array}$ & $\begin{array}{c}\text { Average number } \\
\text { of droplets per } \\
\text { unit volume of } \\
\text { condenser tube, } \\
n_{v, i^{\prime}} \\
\frac{\text { droplets }}{\text { in. }}\end{array}$ & \begin{tabular}{|} 
Average \\
drop- \\
let flux, \\
$\varphi_{\mathrm{i}}=\frac{\mathrm{n}_{\mathrm{t}}}{\mathrm{A}}$ \\
droplets \\
(sec)(in.)
\end{tabular} & $n_{t, i}{ }^{\delta} z, i$ & $\begin{array}{c}\text { Total area } \\
\text { of histogram, } \\
A_{t, i}\end{array}$ \\
\hline 0.00273 & 0.012 & 158 & 8 & 1258 & 1.90 & 0.01459 \\
\hline .00219 & .014 & 217 & 8 & 1728 & 3.04 & .02004 \\
\hline .00224 & .032 & 412 & 16 & 3280 & 6.59 & .03805 \\
\hline .00275 & .054 & 562 & 24 & 4475 & 10.12 & .05191 \\
\hline .00249 & .080 & 738 & 32 & 5876 & 14.76 & .06816 \\
\hline .00349 & .154 & 1119 & 56 & 8909 & 24.62 & .10335 \\
\hline .00248 & .144 & 1311 & 48 & 10438 & 31.46 & .12109 \\
\hline .00324 & .208 & 1811 & 64 & 14419 & 47.09 & .16727 \\
\hline .00446 & .308 & 1150 & 88 & 9156 & 32.20 & .10622 \\
\hline .00792 & .300 & 563 & 80 & 4483 & 16.89 & .05200 \\
\hline .00670 & .256 & 816 & 64 & 6497 & 26.11 & .07537 \\
\hline .00737 & .238 & 517 & 56 & 4116 & 17.58 & .04775 \\
\hline .00594 & .216 & 476 & 48 & $\overline{3} \bar{T} \bar{Y} \bar{y} 0$ & i $\overline{7} .14$ & $.0 \hat{4} 4350$ \\
\hline .00641 & .190 & 501 & 40 & 3989 & 19.04 & .04627 \\
\hline .00763 & .120 & 195 & 24 & 1553 & 7.80 & .01801 \\
\hline .00663 & .168 & 281 & 32 & 2237 & 11.80 & .02595 \\
\hline & & 10827 & 688 & & 288.14 & 0.99999 \\
\hline
\end{tabular}


TABLE III. - Continued. MEASURED

(g) Film 10 (59 in. from inlet); within interval of frames 3000 to 3600 counted from reference point; airplane vapor velocity, 7.17 feet per second.

\begin{tabular}{|c|c|c|c|c|c|c|c|c|c|}
\hline \multirow{3}{*}{$\begin{array}{c}\text { Measured } \\
\text { droplet } \\
\text { diameter, } \\
\delta_{z} \\
\text { in. }\end{array}$} & \multicolumn{4}{|c|}{ Frame } & \multirow{3}{*}{$\begin{array}{l}\text { Measured } \\
\text { frequency, } \\
f, \\
\text { number of } \\
\text { droplets }\end{array}$} & \multirow{3}{*}{$\begin{array}{c}\text { Average } \\
\text { group } \\
\text { range, } \\
\delta_{z} \\
\text { in. }\end{array}$} & \multirow{3}{*}{$\begin{array}{l}\text { Group } \\
\text { mark, } \\
{ }^{6}, \mathbf{i} \text {, } \\
\text { in. }\end{array}$} & \multirow{3}{*}{$\begin{array}{c}\text { Absolute group- } \\
\text { mark frequency, } \\
\qquad \mathfrak{f}_{\mathfrak{i}}, \\
\text { number of } \\
\text { droplets }\end{array}$} & \multirow{3}{*}{$\begin{array}{l}\text { Time for drop- } \\
\text { lets of } i^{\text {th }} \text { group } \\
\text { mark to travel } \\
\text { between two } \\
\text { length marks of } \\
\text { condenser wall, } \\
(\Delta \tau)_{w, i} \\
\text { sec }\end{array}$} \\
\hline & 3000 & 3200 & 3400 & 3600 & & & & & \\
\hline & \multicolumn{4}{|c|}{ Number of droplets } & & & & & \\
\hline 0.017 & 0 & 1 & 0 & 1 & & 0.017 to 0.018 & 0.018 & 1 & $-\cdots--$ \\
\hline .018 & 2 & 1 & 2 & 0 & 1 & 0.0स & 0.020 & & \\
\hline .019 & 1 & 1 & 3 & 0 & 1 & 0019 to 0090 & & & \\
\hline .020 & 2 & 1 & 0 & 2 & 1 & 0.019 to 0.020 & .020 & 2 & $\cdots$ \\
\hline .021 & 1 & 0 & 3 & 0 & & 0.021 to 0.022 & 022 & 3 & 0.01034 \\
\hline .022 & 2 & 3 & 1 & 3 & 2 & $0.02800 .02 a$ & .022 & 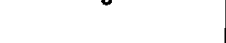 & 0.01007 \\
\hline .023 & 3 & 4 & 2 & 2 & 3 & 0.023 to 0.024 & 024 & 5 & 01577 \\
\hline .024 & 2 & 1 & 1 & 3 & 2 & 0.020100 .064 & .024 & $\mathbf{0}$ & . \\
\hline .025 & 3 & 4 & 2 & 1 & 3 & 0.025 to 0026 & 026 & 7 & 01441 \\
\hline .026 & 3 & 5 & 4 & 4 & 4 & 0.000000 .020 & & & \\
\hline .027 & 2 & 3 & 4 & 3 & 3 & 0027 to 0028 & 028 & 9 & 01500 \\
\hline .028 & 6 & 7 & 5 & 7 & 6 & 0.027 to 0.028 & .020 & $y$ & .01000 \\
\hline .029 & 7 & 5 & 4 & 3 & 4 & 0.029 to 0.030 & 030 & 12 & 01556 \\
\hline .030 & 9 & 10 & 6 & 8 & 8 & 0.020 to 0.000 & .00 & 16 & .01000 \\
\hline .031 & 3 & 5 & 4 & 7 & 5 & 0.031 to 0.032 & .032 & 11 & .02115 \\
\hline .032 & 7 & 4 & 5 & 7 & 6 & & & & \\
\hline .033 & 4 & 3 & 2 & 6 & 4 & 0.033 to 0.034 & 034 & 10 & 03382 \\
\hline .034 & 5 & 7 & 7 & 6 & 6 & & & 10 & .00000 \\
\hline .035 & 2 & 4 & 4 & 1 & 3 & 0.035 to 0.036 & .036 & 8 & .03060 \\
\hline .036 & 3 & 8 & 6 & 2 & $5]$ & & & & \\
\hline .037 & 6 & 3 & 2 & 4 & 4 & 0.037 to 0.038 & 038 & 7 & 04153 \\
\hline .038 & 2 & 4 & 3 & 2 & 3 & 0.00100 .000 & & & .07800 \\
\hline .039 & 4 & 1 & 2 & 2 & 2 & 0.039 to 0.040 & .040 & 5 & .03241 \\
\hline .040 & 3 & 2 & 4 & 2 & 31 & & & & \\
\hline .041 & 5 & 1 & 4 & 3 & 3 & 0.041 to 0.042 & 042 & 5 & 02917 \\
\hline .042 & 3 & 1 & 2 & 2 & $2 J$ & & & ( & .02016 \\
\hline .043 & 2 & 2 & 0 & 1 & $1)$ & 0043 to 0044 & 014 & $A$ & \\
\hline .044 & 3 & 2 & 4 & 4 & $3 \int$ & 0.04500 .044 & $.04 \mathbf{4}$ & 4 & .00036 \\
\hline .045 & 2 & 1 & 0 & 3 & $1\}$ & 0.045 to 0.046 & .046 & 3 & 00959 \\
\hline .046 & 3 & 1 & 2 & 2 & $2 \int$ & & & J & .00000 \\
\hline Total & & & & & & & & 82 & \\
\hline
\end{tabular}


DROPLET DISTRIBUTION

test; 1-g environment; vapor quality, 0.03 ; total weight flow rate, 0.0435 pound per second; local mean

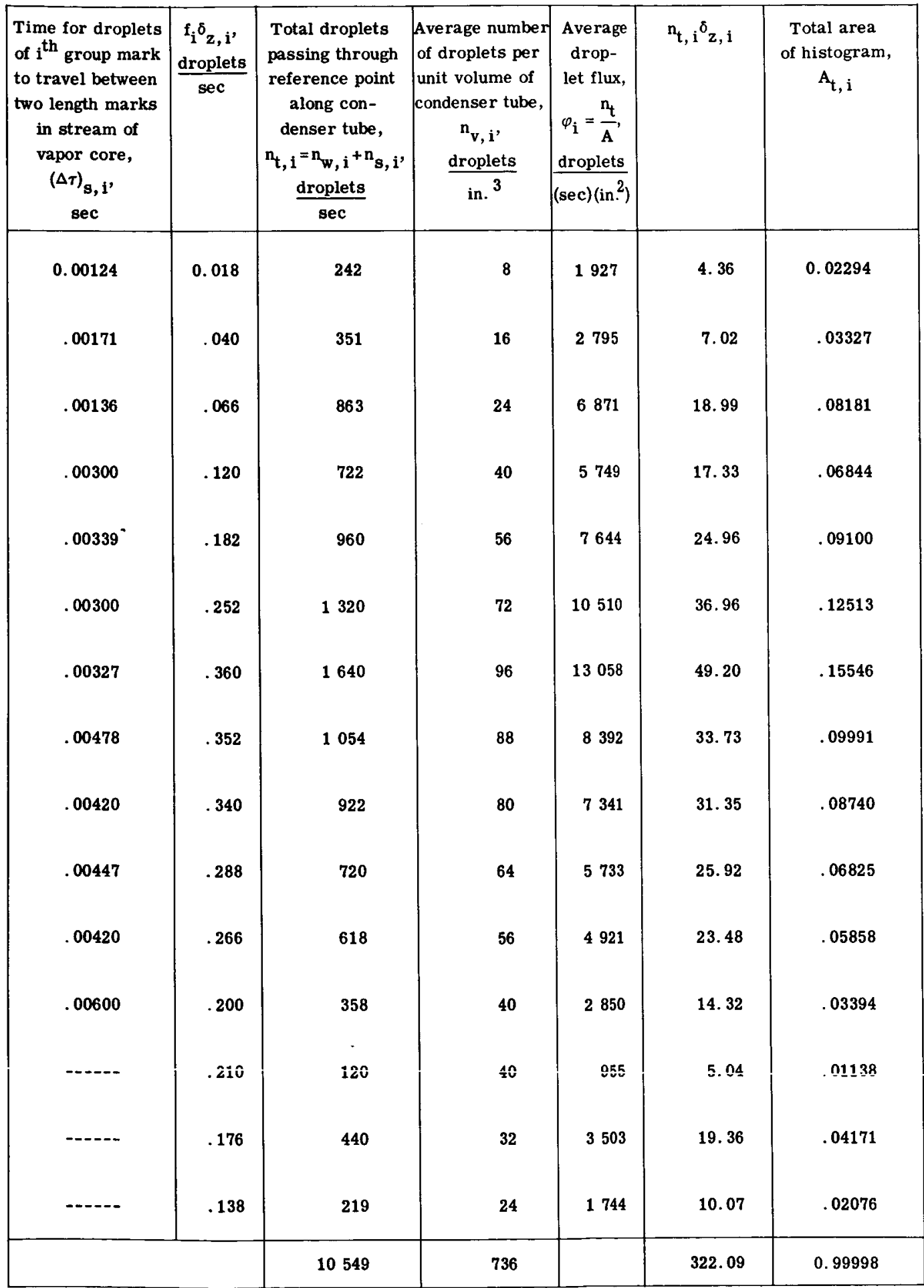


TABLE III. - Continued. MEASURED

(h) Film 6 (60 in. from inlet); within interval of frames 3200 to 3800 counted from reference point; airplane local mean vapor velocity, 5.94 feet per second

\begin{tabular}{|c|c|c|c|c|c|c|c|c|c|}
\hline \multirow{3}{*}{$\begin{array}{c}\text { Measured } \\
\text { droplet } \\
\text { diameter, } \\
{ }^{6} \mathrm{z} \\
\text { in. }\end{array}$} & \multicolumn{4}{|c|}{ Frame } & \multirow{3}{*}{$\begin{array}{c}\text { Measured } \\
\text { frequency, } \\
\mathrm{f}, \\
\text { number of } \\
\text { droplets }\end{array}$} & \multirow{3}{*}{$\begin{array}{c}\text { Average } \\
\text { group } \\
\text { range, } \\
\delta_{z} \text { ' } \\
\text { in. }\end{array}$} & \multirow{3}{*}{$\begin{array}{l}\text { Group } \\
\text { mark, } \\
\delta_{z, i}, \\
\text { in. }\end{array}$} & \multirow{3}{*}{$\begin{array}{c}\text { Absolute group- } \\
\text { mark frequency, } \\
f_{i} \\
\text { number of } \\
\text { droplets }\end{array}$} & \multirow{3}{*}{$\begin{array}{l}\text { Time for drop- } \\
\text { lets of ith group } \\
\text { mark to travel } \\
\text { between two } \\
\text { length marks of } \\
\text { condenser wall, } \\
(\Delta \tau)_{w, 1} \\
\text { sec }\end{array}$} \\
\hline & 3200 & 3400 & 3600 & 3800 & & & & & \\
\hline & \multicolumn{4}{|c|}{ Number of droplets } & & & & & \\
\hline 0.017 & 1 & 0 & 1 & 0 & $0)$ & 0.017 to 0.018 & 0.018 & 1 & 0.02400 \\
\hline .018 & 3 & 1 & 0 & 1 & 1 & & & 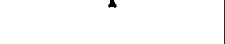 & \\
\hline .019 & 2 & $\mathbf{0}$ & 4 & 3 & 2 & & & & \\
\hline .020 & 1 & 1 & $\mathbf{0}$ & 2 & 1 & 0.019 to 0.020 & .020 & 3 & .02045 \\
\hline .021 & 2 & 3 & 2 & 2 & 27 & & & & \\
\hline .022 & 4 & 5 & 3 & 1 & $3]$ & 0.021 to 0.022 & .022 & 5 & .02655 \\
\hline .023 & 2 & 1 & 0 & 4 & 2 & 0023 to 0094 & & & \\
\hline .024 & 5 & 3 & 5 & 4 & 4 & 0.023 to 0.024 & .024 & 6 & .01857 \\
\hline .025 & 7 & 4 & 6 & 2 & $4)$ & & & & \\
\hline .026 & 4 & 7 & 6 & 8 & 6 & 0.025 to 0.026 & .026 & 10 & .02449 \\
\hline .027 & 3 & 6 & 4 & 4 & 4 & & & & \\
\hline .028 & $\theta$ & 5 & 11 & 8 & 8 & 0.027 to 0.028 & .028 & 12 & .02509 \\
\hline .029 & 3 & 3 & 2 & 4 & 3 & 0.029 to 0.030 & 030 & 8 & $0971 ?$ \\
\hline .030 & 8 & 5 & 3 & 3 & 5 & 0.02000 .000 & .000 & 0 & .02112 \\
\hline .031 & 3 & 2 & 4 & 5 & 3 & & & & \\
\hline .032 & 5 & 3 & 6 & 4 & 4 & 0.031 to 0.032 & .032 & 7 & .01522 \\
\hline .033 & 2 & 1 & 4 & 5 & 3 & 0.033 to 0.034 & .034 & 5 & .01288 \\
\hline .034 & 5 & 1 & 1 & 0 & $2 J$ & & & & \\
\hline .035 & 3 & 2 & 2 & 1 & 2 & 0.035 to 0.036 & 036 & 4 & 01905 \\
\hline .036 & 4 & 0 & 5 & 1 & $2 J$ & & .000 & $z$ & .01000 \\
\hline .037 & 3 & 4 & 2 & 2 & 37 & 0.037 to 0.038 & 038 & 4 & \\
\hline .038 & 2 & 0 & 1 & 1 & $1\}$ & 0.031 to 0.000 & . USO & $\mathbf{4}$ & .01678 \\
\hline .039 & 0 & 3 & 0 & 0 & $1)$ & 0.039 to 0.040 & .040 & 3 & .01957 \\
\hline .040 & 3 & 1 & 2 & 2 & $2 J$ & & & & \\
\hline .041 & 1 & 3 & 0 & 1 & $1\}$ & 0.041 to 0.042 & 042 & 2 & 01558 \\
\hline .042 & 0 & 1 & 0 & 2 & $1 J$ & & & & .01000 \\
\hline Total & & & & & & & & 70 & \\
\hline
\end{tabular}


DROPLET DISTRIBUTION

test; zero-gravity environment; vapor quality, 0.03 ; total weight flow rate, 0.0315 pound per second;

\begin{tabular}{|c|c|c|c|c|c|c|}
\hline $\begin{array}{l}\text { Time for droplets } \\
\text { of } i^{\text {th }} \text { group mark } \\
\text { to travel between } \\
\text { two length marks } \\
\text { in stream of } \\
\text { vapor core, } \\
(\Delta \tau)_{8, i} \\
\text { sec }\end{array}$ & $\begin{array}{c}\begin{array}{c}f_{i}^{\delta} z, i^{\prime} \\
\text { droplets }\end{array} \\
\text { sec }\end{array}$ & $\begin{array}{l}\text { Total droplets } \\
\text { passing through } \\
\text { reference point } \\
\text { along con- } \\
\text { denser tube, } \\
\mathbf{n}_{\mathbf{t}, \mathbf{i}}=\mathrm{n}_{\mathrm{w}, \mathrm{i}}+\mathrm{n}_{\mathrm{s}, \mathrm{i}}, \\
\frac{\text { droplets }}{\text { sec }}\end{array}$ & $\begin{array}{c}\text { Average number } \\
\text { of droplets per } \\
\text { unit volume of } \\
\text { condenser tube, } \\
n_{v, i} \text {, } \\
\frac{\text { droplets }^{3}}{\text { in. }^{3}}\end{array}$ & $\begin{array}{c}\text { Ave rage } \\
\text { drop- } \\
\text { let flux, } \\
\varphi_{\mathrm{i}}=\frac{\mathrm{n}_{\mathrm{t}}}{\mathrm{A}} \\
\text { droplets } \\
\text { (sec)(in.) }\end{array}$ & $\mathrm{n}_{\mathrm{t}, \mathrm{i}} \mathbf{\delta}_{\mathrm{z}, \mathrm{i}}$ & $\begin{array}{c}\text { Total area } \\
\text { of histogram, } \\
A_{t, i}\end{array}$ \\
\hline 0.00702 & 0.018 & 82 & 8 & 653 & 1.48 & 0.01371 \\
\hline .1043 & .060 & 203 & 24 & 1616 & 4.06 & .03395 \\
\hline .00541 & .110 & 483 & 40 & 3846 & 10.63 & .08078 \\
\hline .00882 & .144 & 456 & 48 & 3631 & 10.94 & .07627 \\
\hline .00983 & .260 & 652 & 80 & 5191 & 16.95 & .10905 \\
\hline .00966 & .336 & 784 & 96 & 6242 & 21.95 & .13113 \\
\hline .00426 & .240 & 929 & 64 & 7397 & 27.87 & .15538 \\
\hline .00542 & .224 & 793 & 56 & 6314 & 25.38 & .13263 \\
\hline .00694 & .170 & 521 & 35 & 4148 & 17.71 & .08714 \\
\hline .00769 & .144 & 334 & 32 & 2659 & 12.02 & .05586 \\
\hline .00667 & .152 & 383 & 32 & 3049 & 14.55 & .06406 \\
\hline .00816 & .120 & 239 & 24 & 1903 & 9.56 & .03997 \\
\hline .01860 & .084 & 120 & 16 & 955 & 5.04 &.$\overline{0} \bar{Z} \mathbf{U} \bar{u} \bar{t}$ \\
\hline & & 5979 & 555 & & 178.14 & 1.00000 \\
\hline
\end{tabular}


TABLE III. - Continued. MEASURED

(i) Film 11 (59 in. from inlet); within interval of frames 3200 to 3800 counted from reference point; airplane vapor velocity, 11.60 feet per second

\begin{tabular}{|c|c|c|c|c|c|c|c|c|c|}
\hline \multirow{3}{*}{$\begin{array}{c}\text { Measured } \\
\text { droplet } \\
\text { diameter, } \\
\delta_{z}, \\
\text { in. }\end{array}$} & \multicolumn{4}{|c|}{ Frame } & \multirow{3}{*}{$\begin{array}{l}\text { Measured } \\
\text { frequency, } \\
\text { f, } \\
\text { number of } \\
\text { droplets }\end{array}$} & \multirow{3}{*}{$\begin{array}{l}\text { Average } \\
\text { group } \\
\text { range, } \\
\delta_{z} \text {, } \\
\text { in. }\end{array}$} & \multirow{3}{*}{$\begin{array}{l}\text { Group } \\
\text { mark, } \\
\delta_{z, 1}, \\
\text { in. }\end{array}$} & \multirow{3}{*}{$\begin{array}{l}\text { Absolute group- } \\
\text { mark frequency, } \\
f_{i} \text {, } \\
\text { number of } \\
\text { droplets }\end{array}$} & \multirow{3}{*}{$\begin{array}{l}\text { Time for drop- } \\
\text { lets of } i^{\text {th }} \text { group } \\
\text { mark to travel } \\
\text { between two } \\
\text { length marks of } \\
\text { condenser wall, } \\
(\Delta \tau)_{w, i} \\
\text { sec }\end{array}$} \\
\hline & 3200 & 3400 & 3600 & 3800 & & & & & \\
\hline & \multicolumn{4}{|c|}{ Number of droplets } & & & & & \\
\hline $\begin{array}{r}0.021 \\
.022\end{array}$ & $\begin{array}{l}1 \\
2\end{array}$ & $\begin{array}{l}0 \\
1\end{array}$ & $\begin{array}{l}1 \\
0\end{array}$ & $\begin{array}{l}0 \\
1\end{array}$ & $\begin{array}{l}0 \\
1\end{array}$ & 0.021 to 0.022 & 0.022 & 1 & ------ \\
\hline $\begin{array}{r}.023 \\
.024\end{array}$ & $\begin{array}{l}0 \\
1\end{array}$ & $\begin{array}{l}2 \\
0\end{array}$ & $\begin{array}{l}1 \\
1\end{array}$ & $\begin{array}{l}0 \\
2\end{array}$ & $\begin{array}{l}1 \\
1\end{array}$ & 0.023 to 0.024 & .024 & 2 & 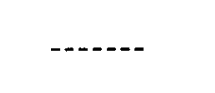 \\
\hline $\begin{array}{r}.025 \\
.026\end{array}$ & $\begin{array}{l}3 \\
4\end{array}$ & $\begin{array}{l}1 \\
2\end{array}$ & $\begin{array}{l}0 \\
5\end{array}$ & $\begin{array}{l}0 \\
1\end{array}$ & $\left.\begin{array}{l}1 \\
3\end{array}\right\}$ & 0.025 to 0.026 & .026 & 4 & $\cdots--\cdots$ \\
\hline $\begin{array}{r}.027 \\
.028\end{array}$ & $\begin{array}{l}3 \\
1\end{array}$ & $\begin{array}{l}2 \\
2\end{array}$ & $\begin{array}{l}1 \\
1\end{array}$ & $\begin{array}{l}1 \\
1\end{array}$ & $\begin{array}{l}2 \\
1\end{array}$ & 0.027 to 0.028 & .028 & 7 & $+\cdots$ \\
\hline $\begin{array}{r}.029 \\
.030\end{array}$ & $\begin{array}{l}3 \\
4\end{array}$ & $\begin{array}{l}1 \\
2\end{array}$ & $\begin{array}{l}0 \\
3\end{array}$ & $\begin{array}{l}0 \\
4\end{array}$ & $\left.\begin{array}{l}1 \\
3\end{array}\right]$ & 0.029 to 0.030 & .030 & 9 & $-\cdots$ \\
\hline $\begin{array}{l}.031 \\
.032\end{array}$ & $\begin{array}{l}0 \\
3\end{array}$ & $\begin{array}{l}3 \\
4\end{array}$ & $\begin{array}{l}2 \\
6\end{array}$ & $\begin{array}{l}3 \\
4\end{array}$ & $\left.\begin{array}{l}2 \\
4\end{array}\right\}$ & 0.031 to 0.032 & .032 & 11 & 0.02933 \\
\hline $\begin{array}{r}.033 \\
.034\end{array}$ & $\begin{array}{l}2 \\
1\end{array}$ & $\begin{array}{l}4 \\
0\end{array}$ & $\begin{array}{l}3 \\
4\end{array}$ & $\begin{array}{l}3 \\
3\end{array}$ & $\begin{array}{l}3 \\
2\end{array}$ & 0.033 to 0.034 & .034 & 8 & .03122 \\
\hline $\begin{array}{l}.035 \\
.036\end{array}$ & $\begin{array}{l}1 \\
2\end{array}$ & $\begin{array}{l}0 \\
3\end{array}$ & $\begin{array}{l}1 \\
2\end{array}$ & $\begin{array}{l}1 \\
1\end{array}$ & $\begin{array}{l}1 \\
2\end{array}$ & 0.035 to 0.036 & .036 & 6 & .04286 \\
\hline $\begin{array}{l}.037 \\
.038\end{array}$ & $\begin{array}{l}2 \\
2\end{array}$ & $\begin{array}{l}0 \\
3\end{array}$ & $\begin{array}{l}1 \\
2\end{array}$ & $\begin{array}{l}0 \\
4\end{array}$ & $\begin{array}{l}1 \\
3\end{array}$ & 0.037 to 0.038 & .038 & 4 & .02254 \\
\hline $\begin{array}{l}.039 \\
.040\end{array}$ & $\begin{array}{l}3 \\
0\end{array}$ & $\begin{array}{l}2 \\
0\end{array}$ & $\begin{array}{l}1 \\
2\end{array}$ & $\begin{array}{l}2 \\
1\end{array}$ & $\left.\begin{array}{l}2 \\
1\end{array}\right)$ & 0.039 to 0.040 & .040 & 3 & .00830 \\
\hline $\begin{array}{r}.041 \\
.042\end{array}$ & $\begin{array}{l}3 \\
0\end{array}$ & $\begin{array}{l}0 \\
0\end{array}$ & $\begin{array}{l}1 \\
2\end{array}$ & $\begin{array}{l}1 \\
2\end{array}$ & $\begin{array}{l}1 \\
1\end{array}$ & 0.041 to 0.042 & .042 & 2 & .00884 \\
\hline $\begin{array}{r}.043 \\
.044\end{array}$ & $\begin{array}{l}0 \\
3\end{array}$ & $\begin{array}{l}1 \\
0\end{array}$ & $\begin{array}{l}1 \\
0\end{array}$ & $\begin{array}{l}1 \\
2\end{array}$ & $\begin{array}{l}1 \\
1\end{array}$ & 0.043 to 0.044 & .044 & 1 & .00734 \\
\hline $\begin{array}{l}.045 \\
.046\end{array}$ & $\begin{array}{l}1 \\
0\end{array}$ & $\begin{array}{l}1 \\
1\end{array}$ & $\begin{array}{l}0 \\
1\end{array}$ & $\begin{array}{l}2 \\
1\end{array}$ & $\left.\begin{array}{l}1 \\
1\end{array}\right\}$ & 0.045 to 0.046 & .046 & 2 & .01143 \\
\hline Total & & & & & & & & 60 & \\
\hline
\end{tabular}


DROPLET DISTRIBUTION

test; 1 -g environment: vapor quality, 0.03 ; total weight flow rate, 0.0324 pound per second; local mean

\begin{tabular}{|c|c|c|c|c|c|c|}
\hline $\begin{array}{l}\text { Time for droplets } \\
\text { of } i^{\text {th }} \text { group mark } \\
\text { to travel between } \\
\text { two length marks } \\
\text { in stream of } \\
\text { vapor core, } \\
(\Delta \tau)_{s, i} \text {, } \\
\text { sec }\end{array}$ & $\begin{array}{c}\begin{array}{c}f_{\mathbf{i}}^{\delta} z, \mathbf{i} \\
\text { droplets }\end{array} \\
\text { sec }\end{array}$ & $\begin{array}{l}\text { Total droplets } \\
\text { passing through } \\
\text { reference point } \\
\text { along con- } \\
\text { denser tube, } \\
\mathrm{n}_{\mathrm{t}, \mathrm{i}}=\mathrm{n}_{\mathrm{w}, \mathrm{i}}+\mathrm{n}_{\mathrm{s}, \mathrm{i}}, \\
\frac{\text { droplets }}{\mathrm{sec}}\end{array}$ & $\begin{array}{c}\text { Average number } \\
\text { of droplets per } \\
\text { unit volume of } \\
\text { condenser tube, } \\
n_{v, i} \text {, } \\
\frac{\text { droplets }}{\text { in. }}\end{array}$ & $\begin{array}{c}\text { Average } \\
\text { drop- } \\
\text { let flux, } \\
\varphi_{\mathrm{i}}=\frac{\mathrm{n}_{\mathrm{t}}}{\mathrm{A}} \\
\text { droplets } \\
\left.\text { (sec)(in. }{ }^{2}\right)\end{array}$ & $n_{t, i^{\delta}, i}$ & $\begin{array}{c}\text { Total area } \\
\text { of histogram, } \\
\mathbf{A}_{\mathbf{t}, \mathbf{i}}\end{array}$ \\
\hline 0.00323 & 0.022 & 62 & 8 & 494 & 1.36 & 0.01298 \\
\hline .00339 & .048 & 118 & 16 & 940 & 2.83 & .02471 \\
\hline .00295 & .104 & 271 & 32 & 2158 & 7.05 & .05674 \\
\hline .00289 & .196 & 485 & 56 & 3862 & 13.58 & .10155 \\
\hline .00292 & .270 & 616 & 72 & 4905 & 18.48 & .12898 \\
\hline .00391 & .352 & 863 & 88 & 6871 & 27.62 & .18070 \\
\hline .00317 & .272 & 710 & 64 & 5653 & 24.14 & .14866 \\
\hline .00300 & .216 & 512 & 48 & 4077 & 18.43 & .10720 \\
\hline .00288 & .152 & 420 & 32 & 3344 & 15.96 & .08794 \\
\hline$-\cdots$ & .120 & 289 & 24 & 2301 & 11.56 & .06051 \\
\hline -.-. & .084 & 181 & 16 & 1441 & 7.60 & .03790 \\
\hline$-\ldots$ & .044 & 109 & 8 & 868 & 4.80 & .02282 \\
\hline --.-- & .092 & 140 & 16 & 1115 & 6. 44 & 02931 \\
\hline & & 4776 & 480 & & 159.85 & 1.00000 \\
\hline
\end{tabular}


TABLE III. - Concluded. MEASURED

(j) Film 14 (47 in. from inlet); within interval of frames 3400 to 3700 counted from reference point; airplane vapor velocity, 43.00 feet per second

\begin{tabular}{|c|c|c|c|c|c|c|c|c|c|}
\hline \multirow{3}{*}{$\begin{array}{c}\text { Measured } \\
\text { droplet } \\
\text { diameter, } \\
\delta_{z} \\
\text { in. }\end{array}$} & \multicolumn{4}{|c|}{ Frame } & \multirow{3}{*}{$\begin{array}{c}\text { Measured } \\
\text { frequency, } \\
\text { f, } \\
\text { number of } \\
\text { droplets }\end{array}$} & \multirow{3}{*}{$\begin{array}{c}\text { Average } \\
\text { group } \\
\text { range, } \\
\delta_{z} \text { ' } \\
\text { in. }\end{array}$} & \multirow{3}{*}{$\begin{array}{l}\text { Group } \\
\text { mark, } \\
\delta_{z, 1} \\
\text { in. }\end{array}$} & \multirow{3}{*}{$\begin{array}{c}\text { Absolute group- } \\
\text { mark frequency, } \\
\text { 'f } \\
\text { number of } \\
\text { droplets }\end{array}$} & \multirow{3}{*}{$\begin{array}{l}\text { Time for drop- } \\
\text { lets of } 1^{\text {th }} \text { group } \\
\text { mark to travel } \\
\text { between two } \\
\text { length marks of } \\
\text { condenser wall, } \\
(\Delta \tau)_{w, 1} \\
\text { sec }\end{array}$} \\
\hline & 3400 & 3500 & 3600 & 3700 & & & & & \\
\hline & \multicolumn{4}{|c|}{ Number of droplets } & & & & & \\
\hline 0.019 & 1 & 0 & 1 & 0 & 01 & 0.019 to 0.020 & 0.020 & 1 & 0.01014 \\
\hline .020 & 1 & 2 & 0 & 0 & 1 & & & & \\
\hline .021 & 0 & 2 & 1 & 1 & 1 & 0.021 to 0.022 & .022 & 2 & .01029 \\
\hline .022 & 1 & 1 & 0 & 2 & 1 & & & & \\
\hline .023 & $\mathbf{0}$ & 1 & 1 & 1 & 1 & 0.023 to 0.024 & .024 & 3 & .01963 \\
\hline .024 & 1 & 2 & 3 & 1 & 2 & & & & \\
\hline .025 & 2 & 2 & 4 & 1 & 2 & 0.025 to 0.026 & .026 & 5 & .03500 \\
\hline .026 & 4 & 3 & 3 & 2 & 3 & 0.025 LO 0.020 & .020 & 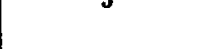 & . Oouro \\
\hline .027 & 0 & 4 & 2 & 3 & 2 & & 028 & 6 & 01359 \\
\hline .028 & 5 & 6 & 4 & 2 & 4 & 0.027 to 0.028 & .028 & 6 & .01358 \\
\hline .029 & 1 & 5 & 4 & 2 & 3 & 0029 to 0030 & 030 & 7 & 01591 \\
\hline .030 & 4 & 2 & 5 & 6 & 4 & 0.02000000 & & & \\
\hline .031 & 7 & 1 & 3 & 4 & 4 & 0.031 to 0.032 & .032 & 8 & .01538 \\
\hline .032 & 3 & 7 & 3 & 4 & 4 & 0.031 to 0.032 & .002 & 0 & .01000 \\
\hline .033 & 2 & 2 & 4 & 3 & 3 & 0033 to 0024 & $024+>$ & 7 & 01898 \\
\hline .034 & 4 & 6 & 4 & 3 & 4 & 0.033 to 0.034 & .034 & $\gamma$ & .01828 \\
\hline .035 & 3 & 2 & 3 & 3 & 3 & 0.035 to 0.036 & .036 & 6 & .01321 \\
\hline .036 & 5 & 4 & 1 & 3 & 3 & & & & \\
\hline .037 & $\mathbf{1}$ & 2 & 2 & 4 & 2 & 0.037 to 0.038 & 038 & 5 & 03571 \\
\hline .038 & 2 & 3 & 3 & 1 & 3 & & & & .00511 \\
\hline .039 & 0 & 1 & 2 & 1 & $1\}$ & 0.039 to 0.040 & 040 & 4 & 03373 \\
\hline .040 & 4 & 2 & 5 & 2 & $3 \int$ & & & & \\
\hline .041 & 0 & 1 & 1 & 3 & 1 & 0 O41 to 0 04a & 014 & 3 & 04200 \\
\hline .047 & 2 & 3 & 2 & 0 & $2 \int$ & 0.041 to 0.044 & $.04 \mathbf{4}$ & 0 & .0zzov \\
\hline Total & & & & & & & & 57 & \\
\hline
\end{tabular}


DROPLET DISTRIBUTION

test: 1-g environment; vapor quality, 0.20; total weight flow rate, 0.0329 pound per second; local mean

\begin{tabular}{|c|c|c|c|c|c|c|}
\hline $\begin{array}{l}\text { Time for droplets } \\
\text { of } i^{\text {th }} \text { group mark } \\
\text { to travel between } \\
\text { two length marks } \\
\text { in stream of } \\
\text { vapor core, } \\
(\Delta \tau))_{8, i} \\
\text { sec }\end{array}$ & $\frac{\begin{array}{c}f_{i}{ }^{\delta} z, i \\
\text { droplets }\end{array}}{\text { sec }}$ & $\begin{array}{l}\text { Total droplets } \\
\text { passing through } \\
\text { reference point } \\
\text { along con- } \\
\text { denser tube, } \\
\mathbf{n}_{t, \mathbf{i}}=\mathbf{n}_{\mathbf{w}, \mathbf{i}}+\mathbf{n}_{\mathrm{s}, \mathbf{i}} \\
\frac{\text { droplets }}{\text { sec }}\end{array}$ & $\begin{array}{l}\text { Average number } \\
\text { of droplets per } \\
\text { unit volume of } \\
\text { condenser tube, } \\
\text { nv,i } \\
\frac{\text { droplets }}{3} \\
\text { in. }\end{array}$ & $\begin{array}{c}\begin{array}{c}\text { Average } \\
\text { drop- }\end{array} \\
\text { let flux, } \\
\varphi_{i}=\frac{n_{t}}{A} \\
\text { droplets } \\
\text { (sec)(in. }\end{array}$ & $n_{t, i}{ }^{\delta} z, i$ & $\begin{array}{c}\text { Total area } \\
\text { of histogram, } \\
\mathbf{A}_{\mathbf{t}, \mathbf{i}}\end{array}$ \\
\hline 0.00390 & 0.020 & 146 & 8 & 1162 & 2.92 & 0.03023 \\
\hline .00522 & .044 & 251 & 16 & 1998 & 5.52 & .05197 \\
\hline .00826 & .072 & 216 & 18 & 1720 & 5.18 & .04472 \\
\hline .00698 & .130 & 315 & 40 & 2508 & 8.19 & .06522 \\
\hline .00756 & .168 & 547 & 48 & 4355 & 15. 32 & .11325 \\
\hline .00442 & .210 & 783 & 56 & 6234 & 23.49 & .16211 \\
\hline .00842 & .256 & 649 & 64 & 5167 & 20.77 & .13437 \\
\hline .00732 & .238 & 555 & 56 & 4419 & 18.87 & .11491 \\
\hline .00522 & .216 & 663 & 48 & 5279 & 23.87 & .13727 \\
\hline .00644 & .190 & 331 & 40 & 2635 & 12.58 & .06853 \\
\hline .00851 & .160 & 224 & 32 & 1783 & 8.96 & .04638 \\
\hline .00900 & .132 & 150 & 24 & 1194 & 6.60 & .03106 \\
\hline & & 4830 & 450 & & 152.27 & 1.00002 \\
\hline
\end{tabular}


per second passing through a reference point along the condenser tube was obtained from the relation

$$
\begin{aligned}
n_{t, i} & =n_{w, i}+n_{s, i} \\
& =f_{i}\left(\frac{\text { Percentage of drops on wall }}{\Delta \tau_{w, i}}+\frac{\text { Percentage of drops in stream of vapor core }}{\Delta \tau_{s, i}}\right)
\end{aligned}
$$

where, $\Delta \tau_{\mathrm{w}, \mathrm{i}}$ and $\Delta \tau_{\mathrm{s}, \mathrm{i}}$ are the time intervals elapsed for a droplet size of the $\mathrm{i}^{\text {th }}$ group mark to travel between the two length marks on a condenser wall and in a stream of vapor core, respectively.

The number of droplets obtained from equation (4) is called absolute frequency per second of the $i^{\text {th }}$ interval of group mark of the droplet size. In order to obtain the number of droplets per unit volume of a condenser tube, the absolute group-mark frequency $f_{i}$ of a given droplet size was divided by the condenser volume confined between the two length marks. Thus,

$$
\mathrm{n}_{\mathrm{v}, \mathrm{i}}=\frac{\mathrm{f}_{\mathrm{i}}}{\frac{\pi}{4} \mathrm{~d}^{2} \iota}
$$

Dividing the absolute droplet frequency per second (given in eq. (4)) by the crosssectional area of a condenser tube gives the droplet flux (number of droplets per unit cross-sectional area per unit time). Thus,

$\varphi_{i}=\frac{f_{i}}{\frac{\pi}{4} d^{2}}\left(\frac{\text { Percentage of droplets on wall }}{\Delta \tau_{w, i}}+\frac{\text { Percentage of droplets in stream of vapor core }}{\Delta \tau_{w, s}}\right)$

The measured values were reduced by using equations (1) to (6) for each interval of droplet size analyzed. The data, thus, obtained are presented in table III. In order to translate the data given in these tables into graphic form, a system of rectangle coordinates was used, as outlined in reference 5 . The abscissa represents the variable droplet diameter $\delta_{i}$, and the ordinate the absolute frequencies per group interval. The rectangle heights for $n_{t, i}$ and $n_{v, i}$ are defined, respectively, by 


$$
\begin{aligned}
& h_{t, i}=\frac{n_{t, i}}{\Delta \delta \sum_{i=1}^{m} n_{t, i}}=\frac{n_{t, i}}{\Delta \delta N_{t}} \\
& h_{v, i}=\frac{n_{v, i}}{\Delta \delta \sum_{i=1}^{m} n_{v, i}}=\frac{n_{v, i}}{\Delta \delta N_{v}}
\end{aligned}
$$

which denote the absolute frequencies centered at $\delta_{i}$, for instance, and were calculated for each interval of droplet size. When the ordinates of the rectangles $h_{t, i}$ and $h_{v}, i$ were multiplied by the base width $\Delta \delta$, the areas of the respective rectangles were obtained. Thus,

$$
\begin{aligned}
& A_{t, i}=h_{t, i} \Delta \delta=\frac{n_{t, i}}{N_{t}} \\
& A_{v, i}=h_{v, i} \Delta \delta=\frac{n_{v, i}}{N_{v}}
\end{aligned}
$$

The area calculated from these equations represents the relative frequencies centered at $\delta_{i}$. The total calculated area of any histogram approached 1 closely, because the sum of the relative frequencies must be equal to 1 . For example, at the vapor quality of 0.03 , a sum of the areas of all calculated rectangles of the histogram, as tabulated in table III(d), is $\sum_{i=1}^{m} A_{t, i}=0.9995$, whereas the theoretical value, as defined by equation (B4) in appendix B, is 1 . The data calculated by using equations (7) to (9) were tabulated in table $\mathrm{II}$, and the results are plotted in figures 10 to 13, respectively. In these figures, the height of the rectangles, which represents the absolute frequencies, and therefore, the areas which represent the corresponding relative frequencies of the histogram are only proportional to the actual calculated values rather than equal to them.

With a convenient choice of a rectangle width $\Delta \delta$ of 1 unit on the $\delta$-axis, the numerical values obtained for the rectangle heights from equations (7) and (8) are equal to the corresponding rectangle areas, calculated from equations (9) and (10), respectively. 


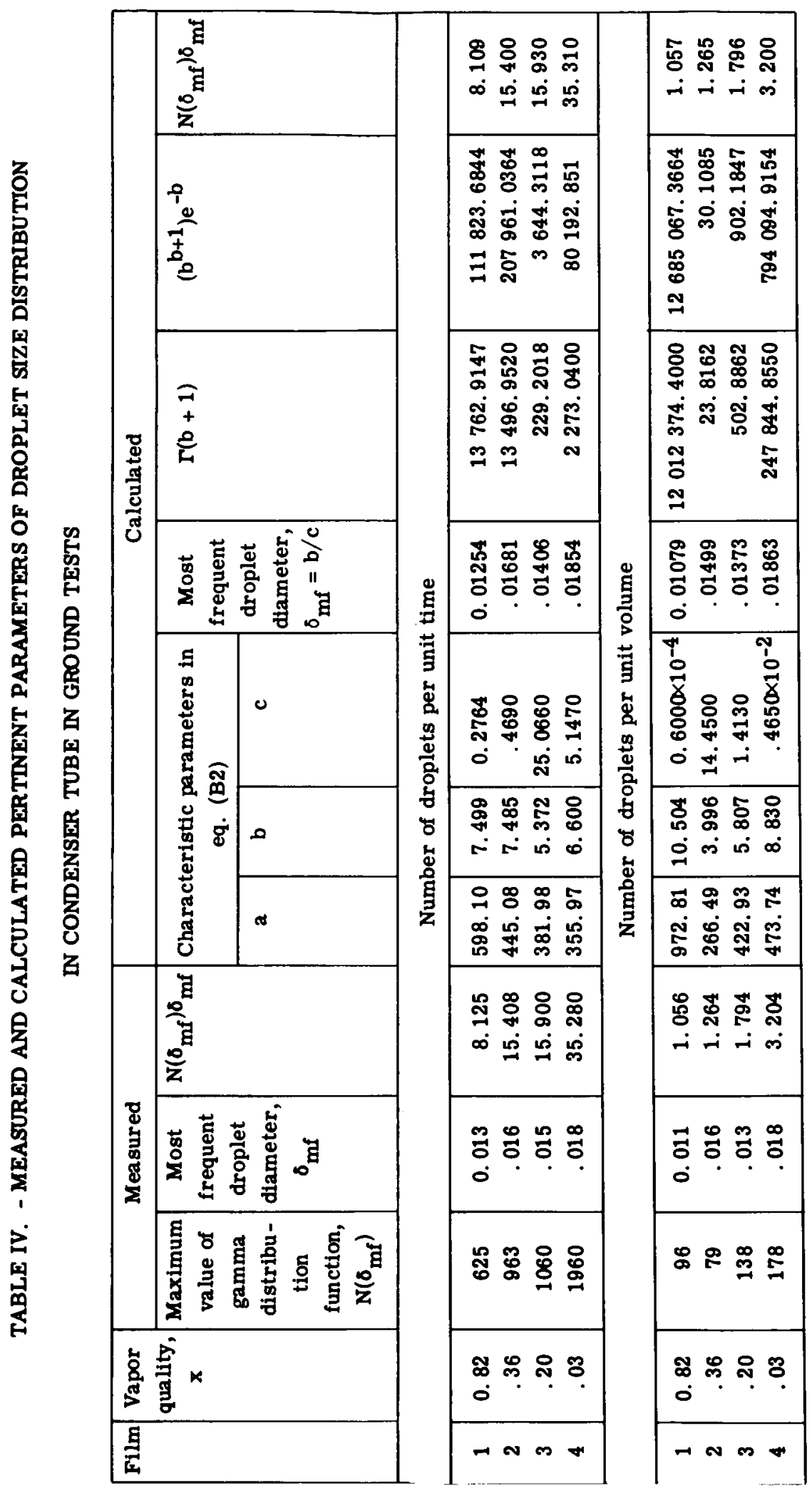


Inspection of histograms of the droplet size distribution showed a marked similarity in shape with gamma distribution given in reference 6 . This suggested the choice of the following three-parameter frequency distribution function,

$$
N(\delta)=C(a \delta)^{b} e^{-a \delta}
$$

which is derived from the gamma probability density function in appendix $B$. The three parameters that comprise a frequency function $N(\delta)$ were determined from the measured data in conjunction with equations (B7) to (B9). A digital computer and a FORTRAN program (Least-Square Estimation of Nonlinear Parameters described in ref. 7, which is available as IBM Share Program No. 1428) were used for this purpose. The numerical values of three characteristic parameters thus determined are tabulated in table IV for ground tests (1-g environment) and in table $V$ for airplane tests (1-g and zero-gravity environments). By substituting these values of the representative parameters into equation (11), a set of two equations, for each vapor quality condition analyzed, may be obtained. Each equation expresses the absolute droplet frequency $N(\delta)$ per unit time and per unit volume, respectively, having a certain diameter $\delta$.

\section{Correlation of Parameters $\mathrm{a}, \mathrm{b}$, and $\mathrm{c}$ and $\delta_{\mathrm{mf}}$ With System Parameters}

In order to obtain a generalized expression for predicting droplet size distribution in a condenser tube, the values of three parameters $a, b$, and $c$, and the most frequent droplet size $\delta_{\mathrm{mf}}$ necessary in describing the gamma distribution law were correlated as a function of system parameters such as mean vapor velocity $V_{m, v}$ and vapor quality $x$. In a range of system parameters investigated $4 \leq \mathrm{V}_{\mathrm{m}, \mathrm{v}} \leq 129$ feet per second and $0.03 \leq \mathrm{x} \leq 0.82$, these parameters were correlated for two conditions of droplet distribution: for expressing the absolute droplet frequency per unit time and per unit volume of a condenser tube.

Ground tests. - In the ground test analysis, the inlet vapor velocity was 141 feet per second, the inlet vapor quality 90 percent or greater, and the total mercury weight flow rate was 0.0385 pound per second. The vapor velocity distribution along the condenser tube is discussed in appendix $\mathrm{C}$.

For a condition where the time function was included in the analysis, the following empirical equations were obtained:

$$
\begin{gathered}
a_{t}=\left(v_{m, v}\right) x^{0.069}+10^{2.559} \\
b_{t}=a_{t, i}\left(\delta_{m f}\right)_{t}
\end{gathered}
$$



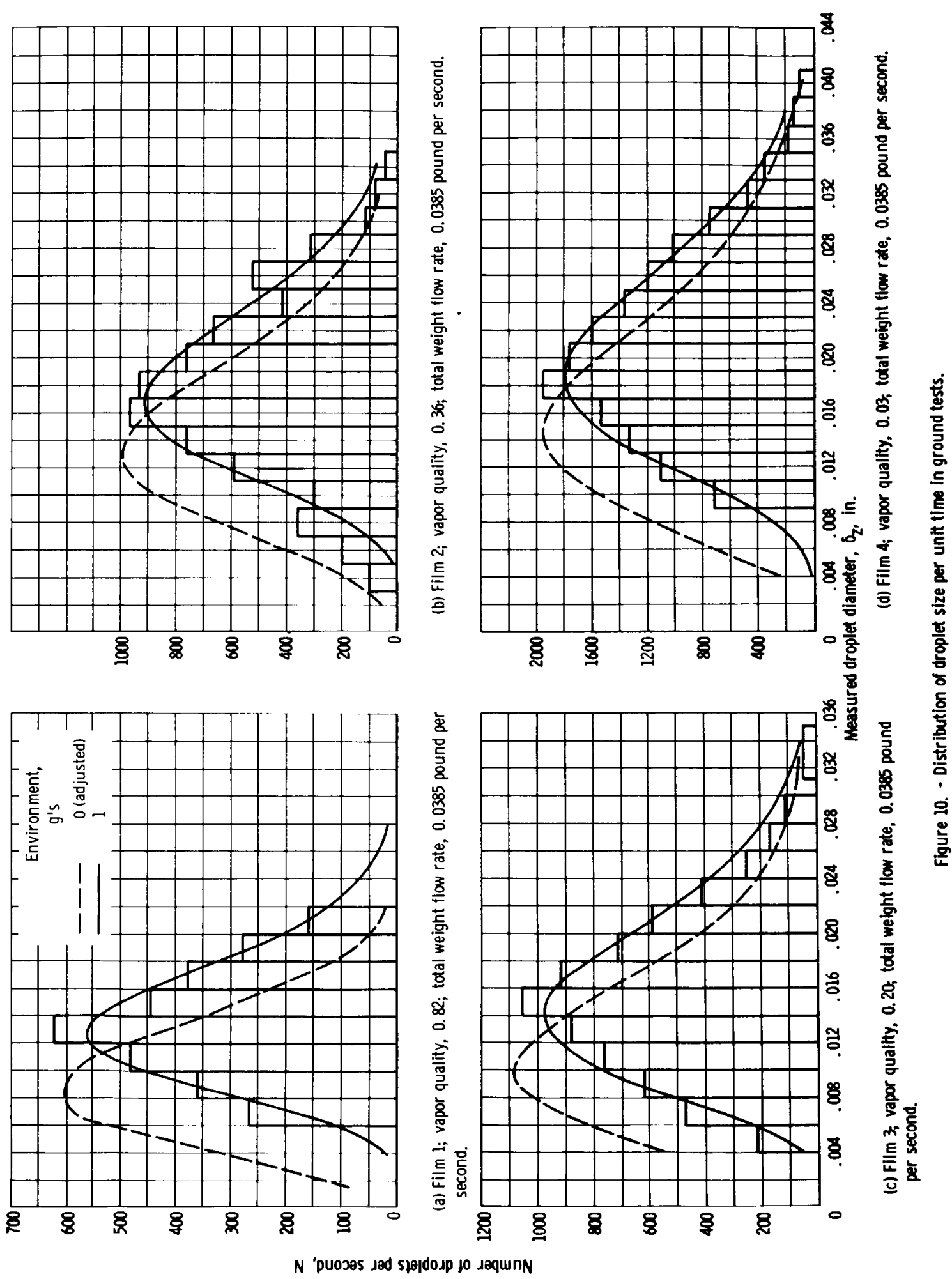


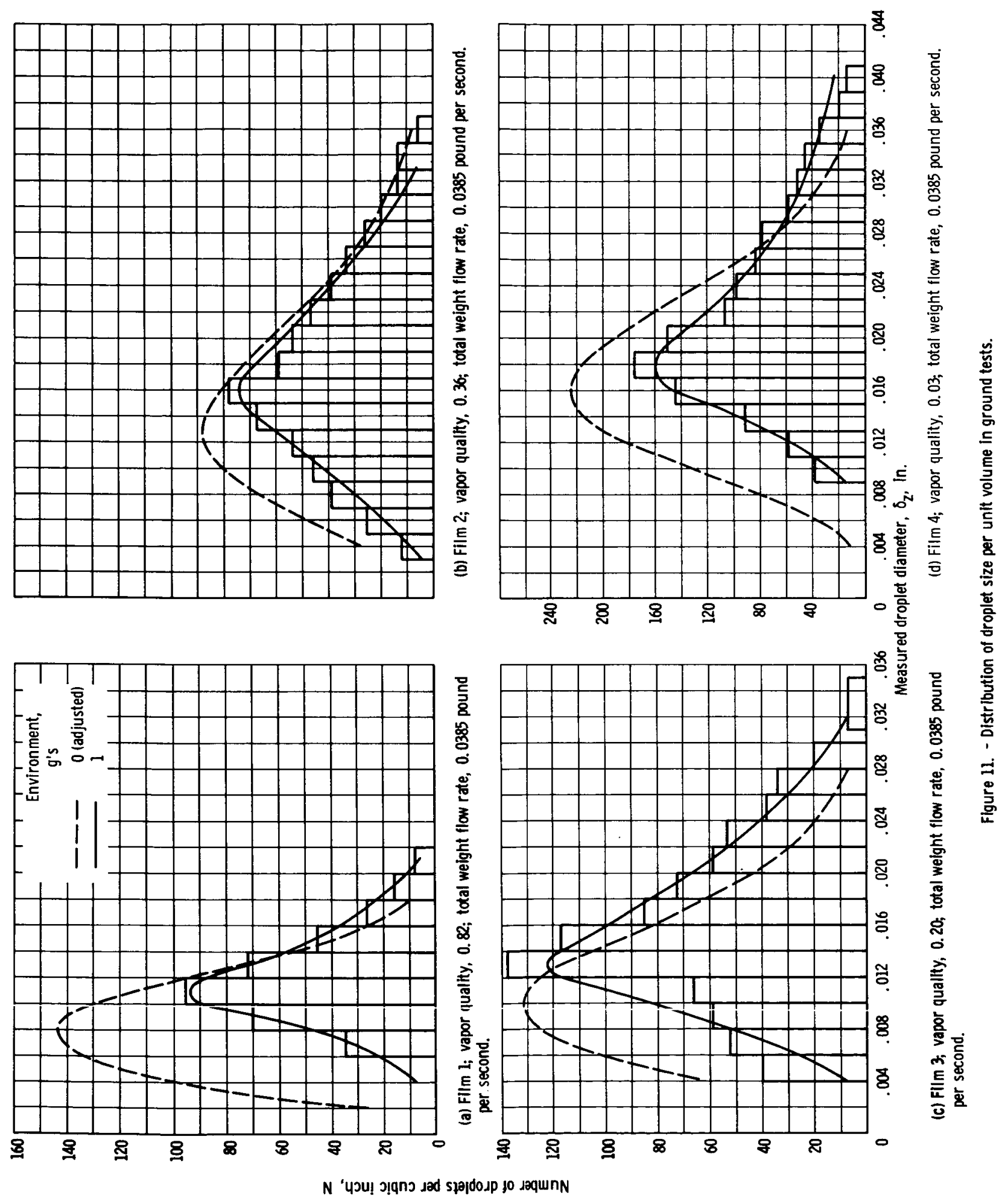




$$
\begin{gathered}
c_{t}=\frac{b_{t}}{\left(\delta_{\mathrm{mf}}\right)_{\mathrm{t}}} \frac{1}{\Gamma\left(b_{\mathrm{t}}+1\right)} \\
\left(\delta_{\mathrm{mf}}\right)_{\mathrm{t}}=5684.620-58.912(1-\mathrm{x}) \mathrm{V}_{\mathrm{m}, \mathrm{v}}
\end{gathered}
$$

For a condition where the volume function was included in the analysis, the correlated parameters were expressed by the following empirical equations:

$$
\begin{gathered}
a_{v}=0.170\left(v_{m, v}-x\right)^{2}-13.328 v_{m, v} x+479.400 \\
b_{v}=a_{v}\left(\delta_{m f}\right)_{v} \\
c_{v}=\frac{b_{v}}{\left(\delta_{f m}\right)_{v}} \frac{1}{\Gamma\left(b_{v}+1\right)} \\
\left(\delta_{m f}\right)_{v}=5684.620-58.912(1-x) v_{m, v}
\end{gathered}
$$

By substituting the values of the correlated parameters into equation (11), the two general empirical equations were obtained for predicting droplet size distribution in a condenser tube, which gave the absolute frequencies of the droplet size distribution (the number of droplets of a given size per second, and the number of droplets of a given size per cubic inch of a condenser tube). These two equations thus obtained are plotted in figures 10 and 11. An accuracy of correlation of the measured data with the empirical equation (11) used, gave a deviation of $1.36 \mathrm{rms}$ percent for the plots shown in figure 10 , and $1.72 \mathrm{rms}$ percent for the plots shown in figure 11.

Airplane tests. - The airplane tests were conducted in 1-g and zero-gravity environments. In these tests, the vapor inlet velocity ranged from 165 to 235 feet per second, the vapor inlet quality was 90 percent or greater, and the total mercury weight flow rate ranged from 0.0315 to 0.0435 pound per second. In the airplane tests, the films of a condensing mercury vapor flow were taken simultaneously at two different locations (two high-speed cameras were available) for vapor quality conditions of 0.20 and 0.03 .

The field of view of the camera in the airplane installation was limited to about 4 inches of the condensation tube. Therefore, the droplet magnification from film to the analyzer screen, depending on magnification lens used, ranged from 1.4 to 2.2. Because of the small magnification factor, small droplets were not visible in the condenser tube; 


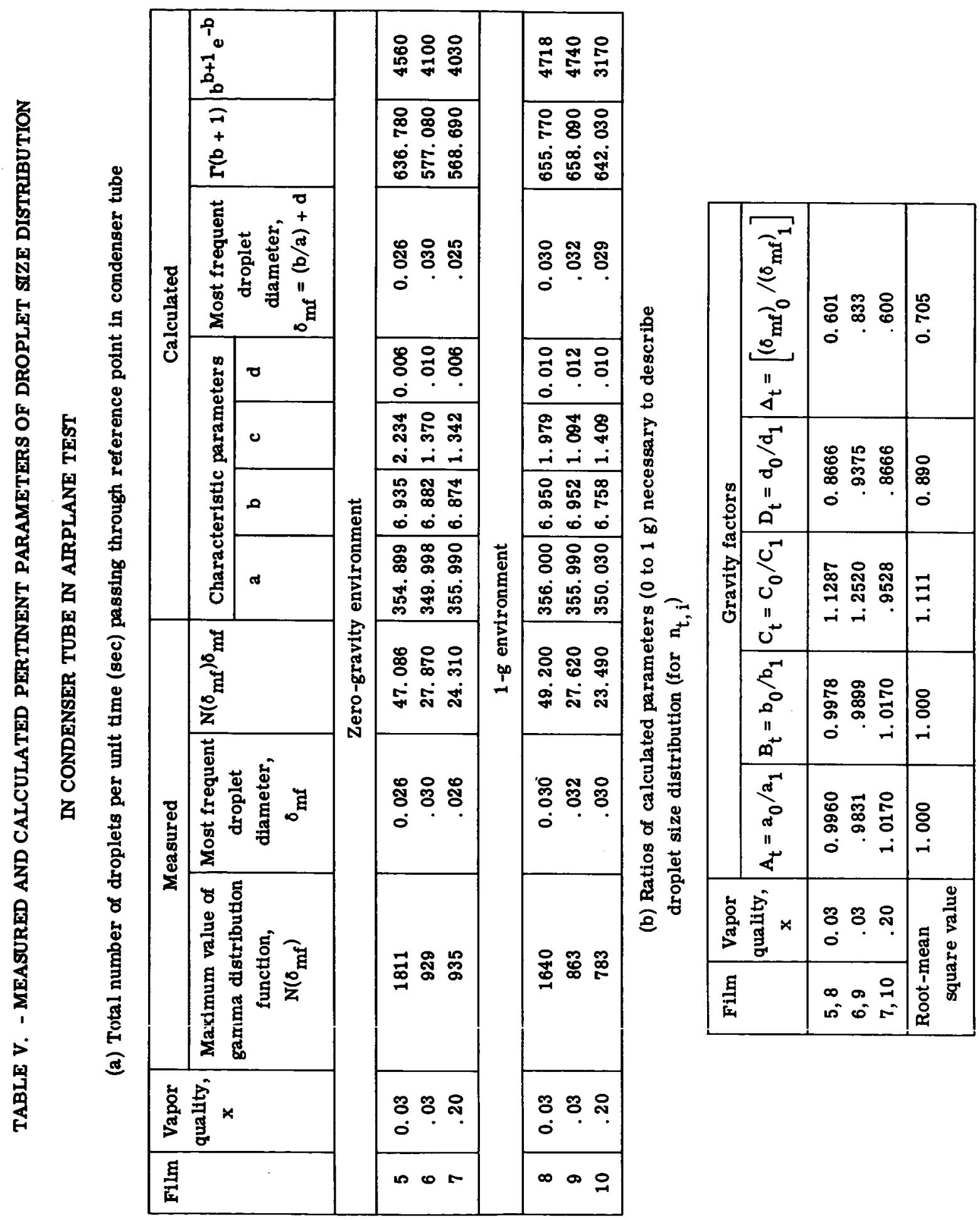




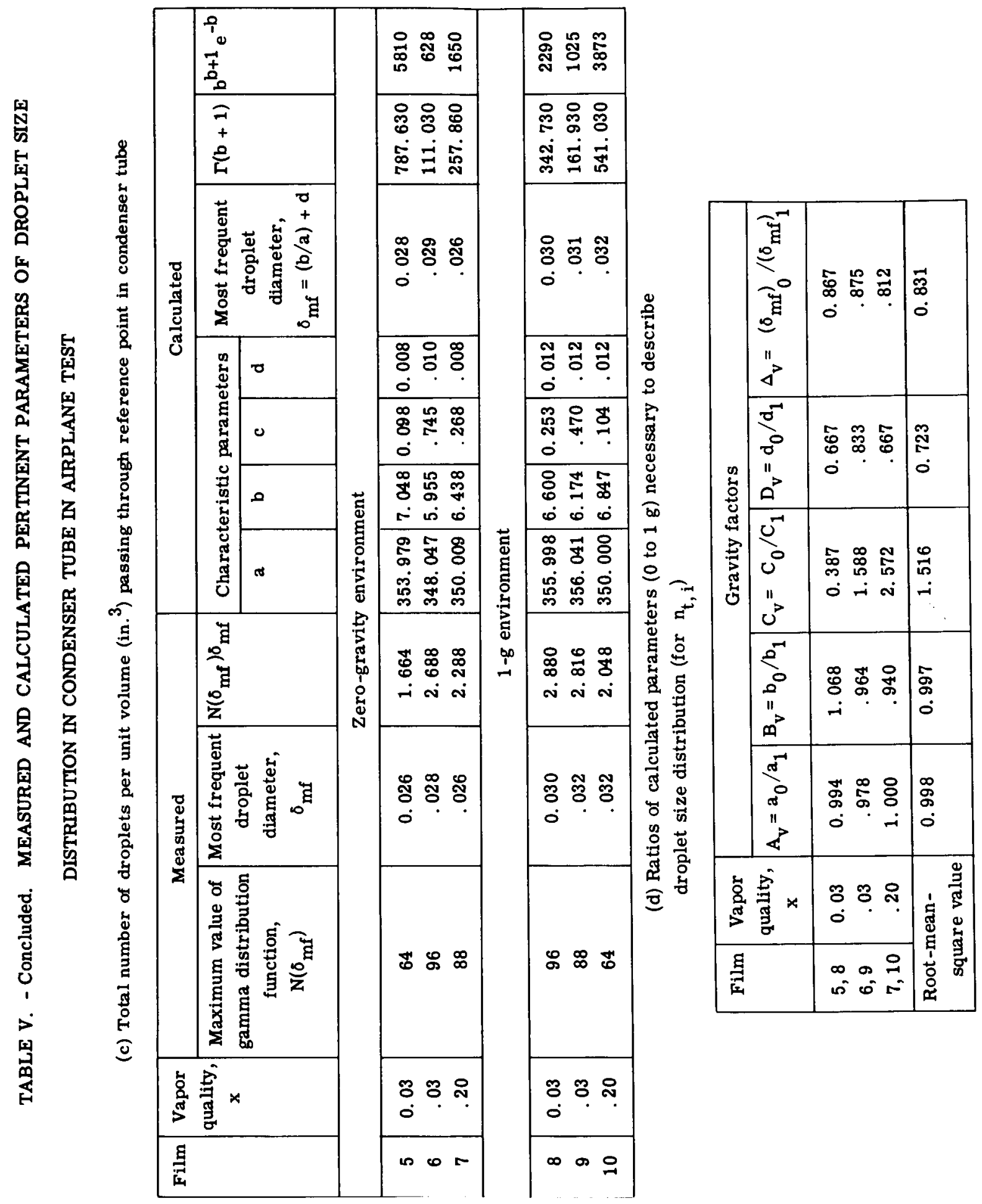


therefore, the droplet size distribution obtained from the airplane tests indicates mainly the effect of gravity level rather than an actual droplet size distribution.

The numerical values of the three characteristic parameters $a, b$, and $c$ were determined for each test run and gravity level investigated from the test data. The results obtained are tabulated in table $\mathrm{V}$ for 1 -g and zero-gravity environments for expressing the absolute droplet frequency per unit time and the number of droplets per unit volume of a condenser tube. By substituting these values into equation (11), two empirical equations were obtained for each test run, which gave the absolute frequencies of the droplet size distribution, the number of droplets of a given size per second, and the number of droplets of a given size per cubic inch of condenser tube. These two equations are plotted in figures 12 and 13 for 1 -g and zero-gravity environments, respectively. The accuracy of the measured data relative to the empirical equation (eq. (11)) obtained in the airplane tests ranged from 1.83 to $2.40 \mathrm{rms}$ percent.

Effect of gravity. - The effect of gravity level on the droplet size distribution in a condenser tube can be seen from figures 12 and 13. In zero gravity, mercury droplets were more uniformly dispersed through the vapor phase. In addition to this, observation indicated that in the vapor stream and on the condenser wall, the droplets were moving toward the liquid interface, with more uniform axial velocity (less turbulent) in zero gravity than in a $1-\mathrm{g}$ environment. On the average, the size of droplets were somewhat larger and lesser in number in a $1-\mathrm{g}$ than in a zero-gravity environment. For example, for the same total weight flow rate and vapor quality condition, the most frequent droplet diameter in $1 \mathrm{~g}$ was $0.030 \mathrm{inch}$, and in zero gravity 0.026 inch (see fig. 14). In zero gravity, droplet distribution was more symmetric about its most frequent droplet size than in a $1-\mathrm{g}$ environment. The larger droplets, in a 1-g environment, on the tube bottom resulted from the effect of agglomeration, as the droplets migrated toward the bottom of the tube. Figure 15 indicates the degree to which gravity affects the flow regime. At $1 \mathrm{~g}$, the droplets on the wall have flowed to the bottom of the tube, agglomerating into larger droplets. In zero-gravity environment, the droplets on the wall have no tendency to migrate and collect in any one part of the tube wall, rather they flow parallel to the tube axis.

As can be seen from figures 12 and 13, in the range of mercury weight flow rate and vapor quality conditions studied, the pattern of droplet size distribution varied in the same fashion, approximately, from zero gravity to a 1 -g environment. Furthermure, examination of table $\mathrm{V}$ indicates that the ratios $(\mathscr{A}, \mathscr{B}, \mathscr{C}$, and $\Delta)$ of the parameter necessary to describe the droplet size distribution law for zero gravity to that of a $1-\mathrm{g}$ environment varied little from one test condition to the other. Therefore, in order to transpose the droplet size distribution from 1-g to zero-gravity environment, it is necessary to correct the parameters a, b, c, and $\delta_{\mathrm{mf}}$ of $1-\mathrm{g}$ environment by the proper gravity factors $\mathscr{A}, \mathscr{B}, \mathscr{C}$, and $\Delta$, respectively. 


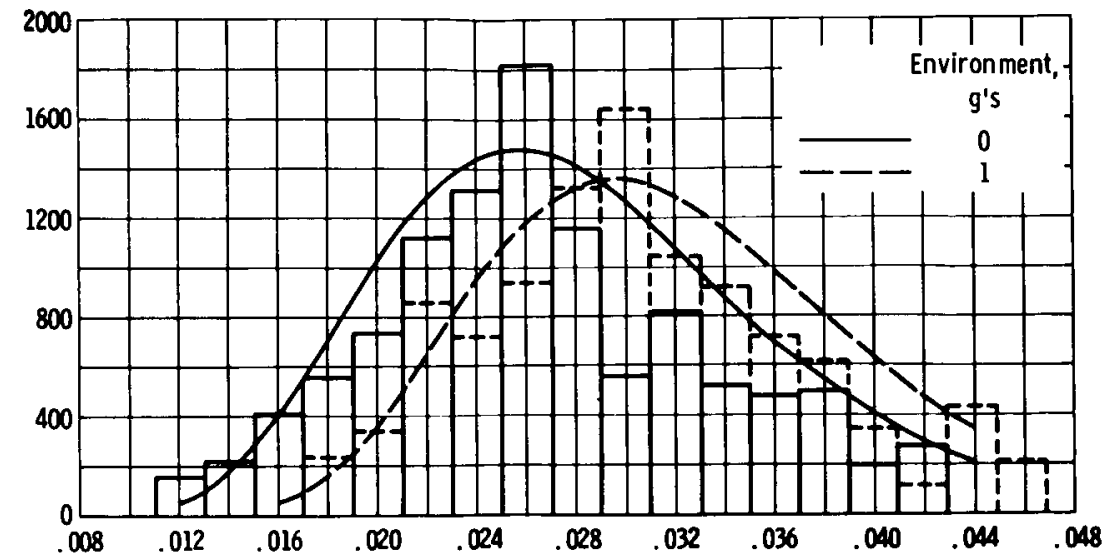

(a) Films 5 and 10, vapor quality, 0.03, total weight flow rate, 0.0435 pound per second.

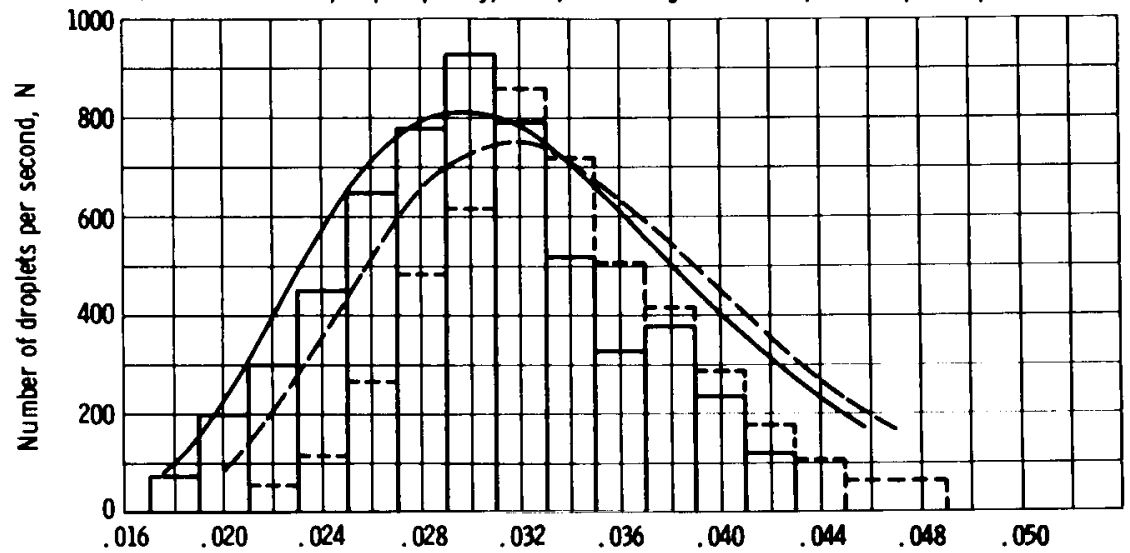

(b) Films 6 and 9; vapor quality, 0.03; total weight flow rate, 0.032 pound per second.

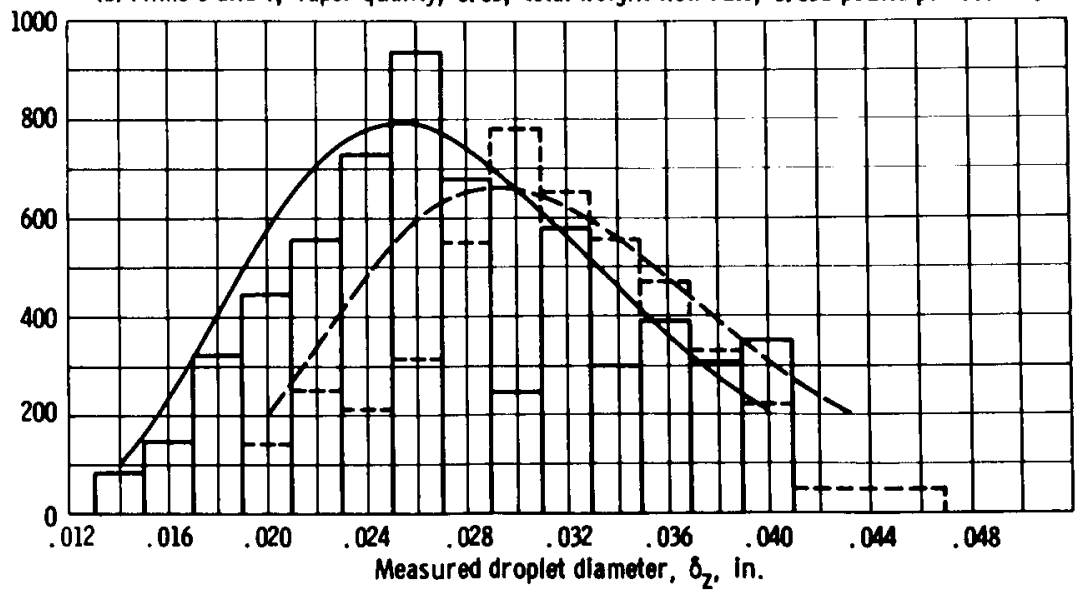

(c) Fllms 7 and 10 ; vapor quality, 0.02; total weight flow rate, 0.0329 pound per second.

Figure 12. - Distribution of droplet size per unit time in airplane tests. 


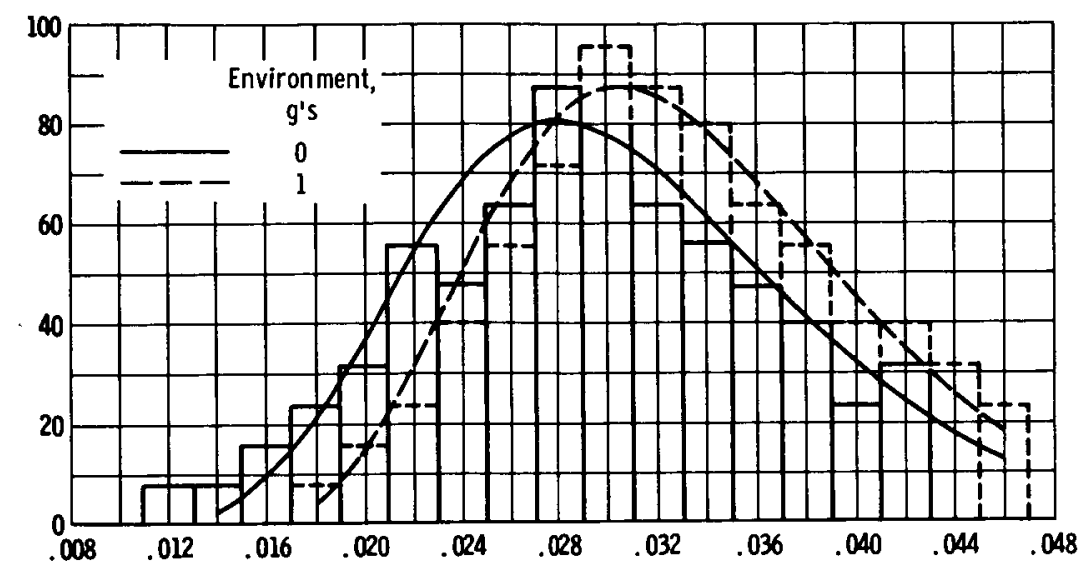

(a) Films 5 and 10, vapor quality, 0.03 ; tokal weight flow rate, 0.0435 pound per second.

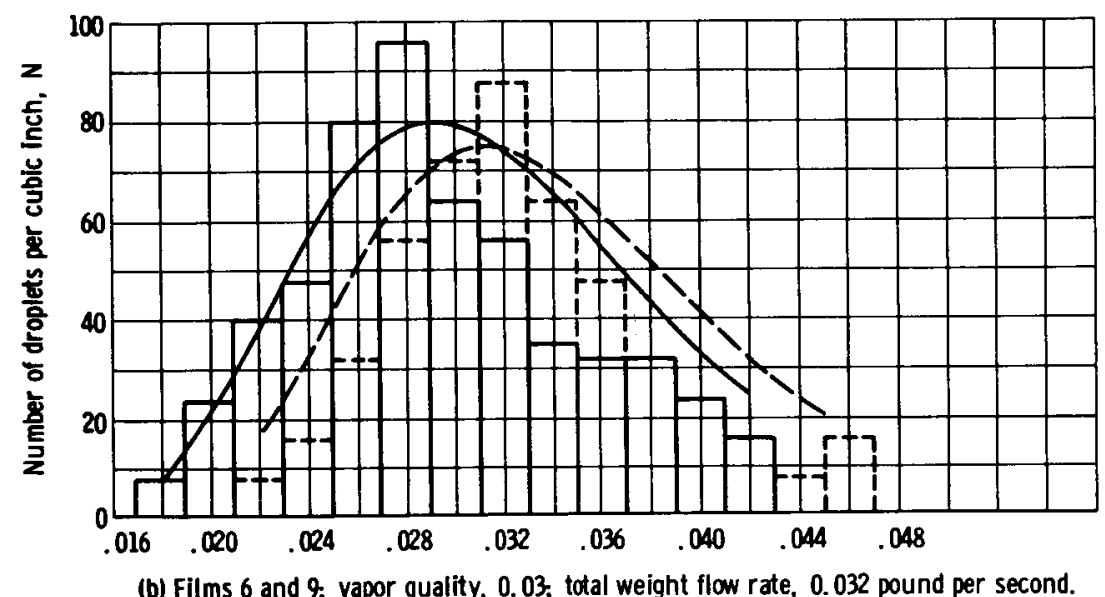

(b) Films 6 and 9; vapor quality, 0.03 ; total weight flow rate, 0.032 pound per second.

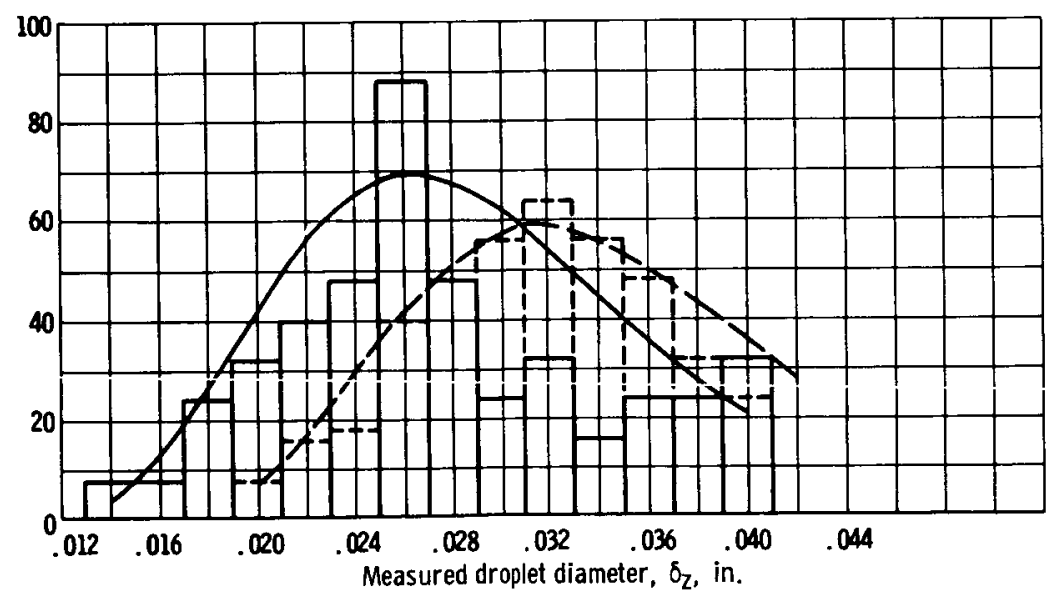

(c) Films 7 and 10, vapor quality, 0.20 , total weight flow rate, 0.0329 pound per second.

Figure 13. - Distribution of droplet size per unit volume in airplane tests. 


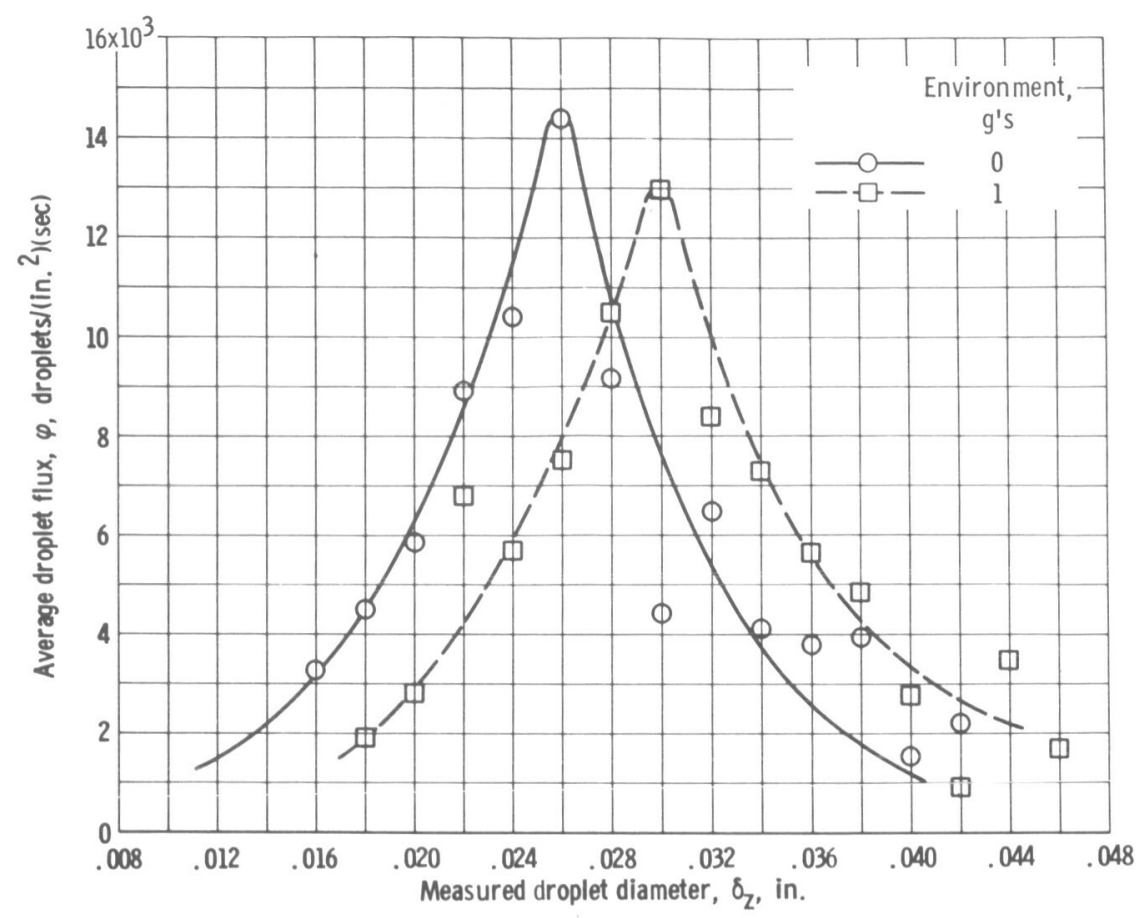

Figure 14. - Average distribution of droplet flux obtained from films 5 and 10 . Airplane test; total weight flow rate, $\mathbf{0 . 0 4 3 5}$ pound per second; vapor quality, 0.03 .

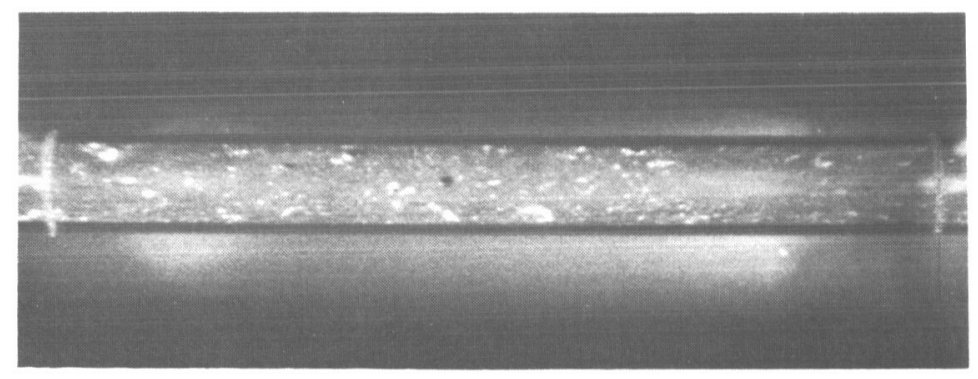

(a) Zero gravity.

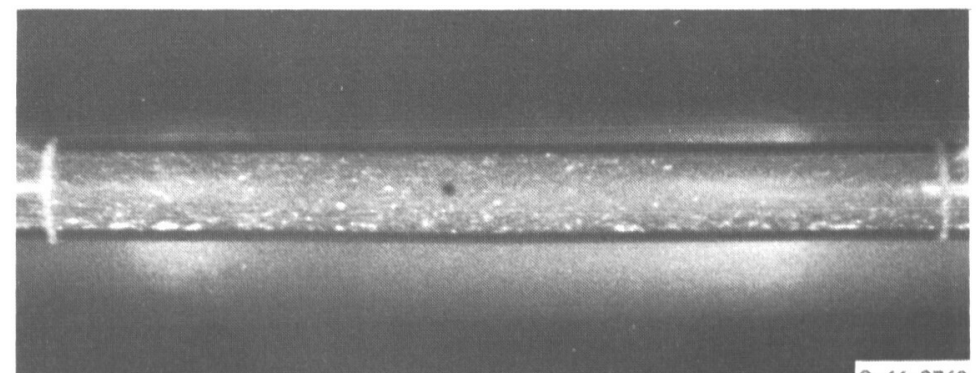

C- $66-3769$

Flow direction

(b) $1 \mathrm{~g}$.

Figure 15. - Condensing mercury vapor flow in vapor region. Vapor quality condition 0.20 , total weight flow rate, 0.0435 pound per second; tube diameter, 0.400 inch. 


\section{Modification of Ground Test Data to Obtain Zero-Gravity}

\section{Droplet Distribution}

In order to define a droplet size distribution function in zero-gravity environment from the ground test data, the parameters a, b, c, and $\delta_{\mathrm{mf}}$ expressed by equations (12) to (18), necessary to describe the droplet size distribution law on the ground, were corrected by the gravity factors $\mathscr{A}, \mathscr{S}, \mathscr{C}$, and $\Delta$, respectively. The values of these factors are listed in table $\mathrm{V}$ (pp. 41 and 42 ).

By substituting the values of the corrected parameters into equation (11), two empirical equations were obtained for predicting droplet size distribution in a condenser tube in zero-gravity environment when the ground test data are known. One of the equations expressed the number of droplets of a given size per second, and the other, the number of droplets of a given size per cubic inch of a condenser tube. These two equations, thus obtained, are plotted in figures 10 and 11.

Droplet flux. - The expression for droplet flux, given by equation (6), defines the number of droplets of a given size crossing through the cross-sectional area of a condenser tube per unit area per unit time.

Ground tests. - Equation (6) was used to calculate the droplet flux for each interval of the droplet size analyzed. The data obtained are tabulated in table III (pp. 13 to 33), and the results are presented in figures 16 and 17 . In figure 16, the droplet flux obtained by cross-plotting figure 14 for the three most frequent droplet diameters $(0.010,0.015$, and $0.020 \mathrm{in.}$ ) in a condenser tube is plotted as a function of a droplet diameter for various

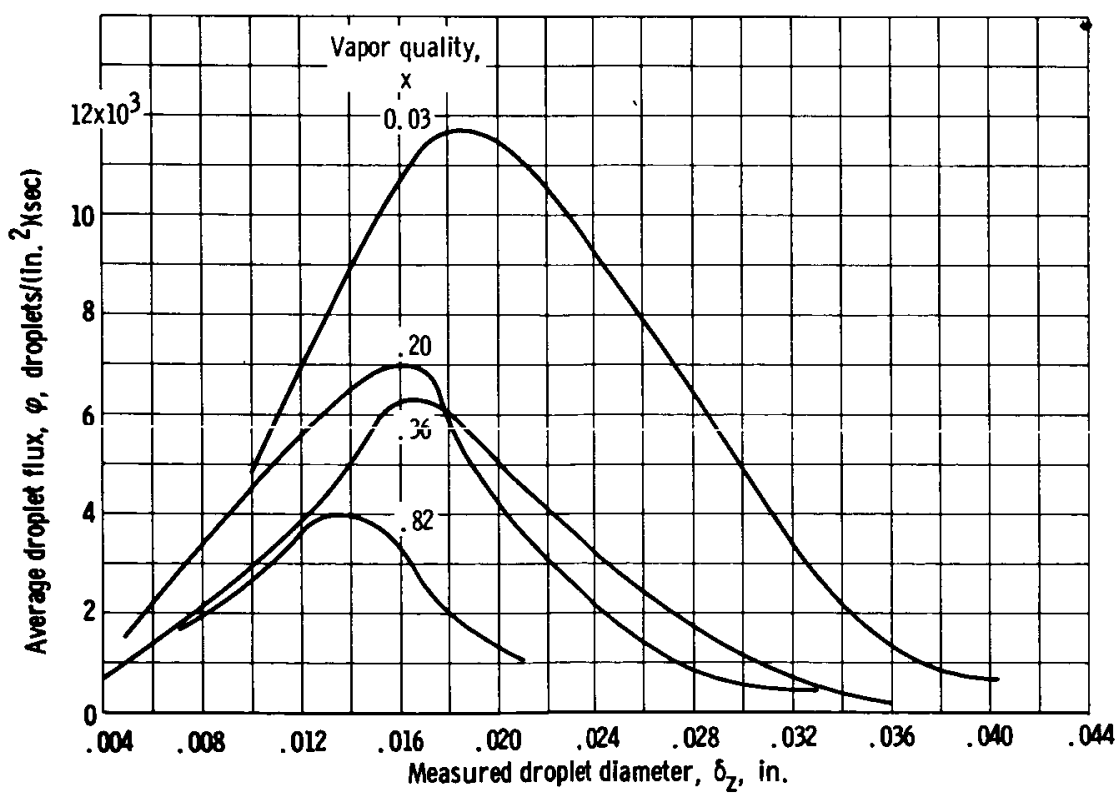

Figure 16. - Average distribution of droplet flux obtained from films 1 to 4 . Ground test; total weight flow rate, 0.0385 pound per second. 


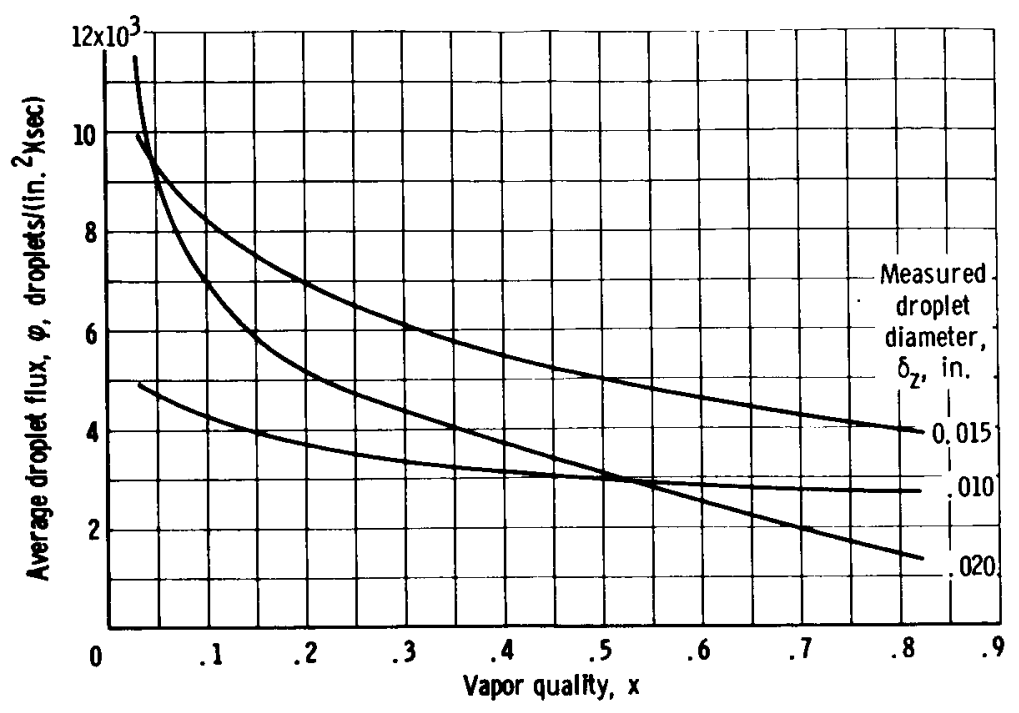

Figure 17. - Average distribution of droplet flux as function of vapor quality. Ground test; total weight flow rate, 0.0385 pound per second.

vapor qualities. As shown in this plot, the droplet flux increases very fast with a decrease in vapor quality. For example, an increase in the droplet flux of the 0.015-inchdiameter droplet, with a decrease in vapor quality is shown in the following table:

\begin{tabular}{|c|c|}
\hline $\begin{array}{c}\text { Vapor quality, } \\
\mathbf{x}\end{array}$ & $\begin{array}{c}\text { Droplet flux, } \\
\varphi,\end{array}$ \\
& $\frac{\text { Number of droplets }}{\left.\text { (in. }^{2}\right)(\mathrm{sec})}$ \\
\hline 0.80 & 2700 \\
.50 & 5000 \\
.03 & 9900 \\
\hline
\end{tabular}

Effect of gravity on droplet flux. - In a range of mercury weight flow rate and the vapor quality conditions investigated, equation (6) was used to calculate the average droplet flux for the airplane tests. The data obtained for 1-g and zero-gravity environments are tabulated in tables $\mathrm{III}(\mathrm{e})$ to (i)(pp. 22 to 31 ).

For the same mercury weight flow rate and vapor quality conditions investigated, the effect of gravity environment on the droplet flux in a condenser tube is shown in figure 16. The peak of the droplet flux is shown to have shifted somewhat toward the larger droplet size in a 1-g environment. In general, in a 1-g environment, the droplets were larger and moved with slower axial velocity in comparison with the droplets studied in zerogravity environment.

Maximum stable droplet size. - From the visual study of two-phase mercury vapor low in a condenser tube (both in ground and airplane tests), it was observed that at a 
TABLE VI. - ROOT-MEAN-SQUARE VALUES

OF MEASURED DROPLET PARAMETERS

IN 1 -g ENVIRONMENT

\begin{tabular}{|c|c|c|c|c|}
\hline $\begin{array}{c}\text { Vapor } \\
\text { quality, } \\
\mathrm{x}\end{array}$ & $\begin{array}{c}\text { Droplet } \\
\text { height, } \\
\delta_{\mathrm{y}}, \\
\text { in. }\end{array}$ & $\begin{array}{c}\text { Droplet } \\
\text { diameter, } \\
\delta_{\mathrm{z}}, \\
\text { in. }\end{array}$ & $\begin{array}{c}\text { Maximum } \\
\text { stable } \\
\text { diameter, } \\
\left(\delta_{\mathrm{max}}\right)_{\mathrm{x}} \\
\text { in. }\end{array}$ & $\begin{array}{c}\text { Critical } \\
\text { diameter, } \\
\left(\delta_{\mathrm{c}}\right)_{\mathrm{x}}, \\
\text { in. }\end{array}$ \\
\hline 0.82 & $\begin{array}{r}0.0110 \\
.36\end{array}$ & $\begin{array}{r}.0121 \\
.0134\end{array}$ & $\begin{array}{r}.0170 \\
.0182\end{array}$ & $\begin{array}{r}.0051 \\
.0190\end{array}$ \\
.03 & .0125 & .0178 & .0215 & .0093 \\
.0144 & .0187 & .0218 & .0120 \\
\hline
\end{tabular}

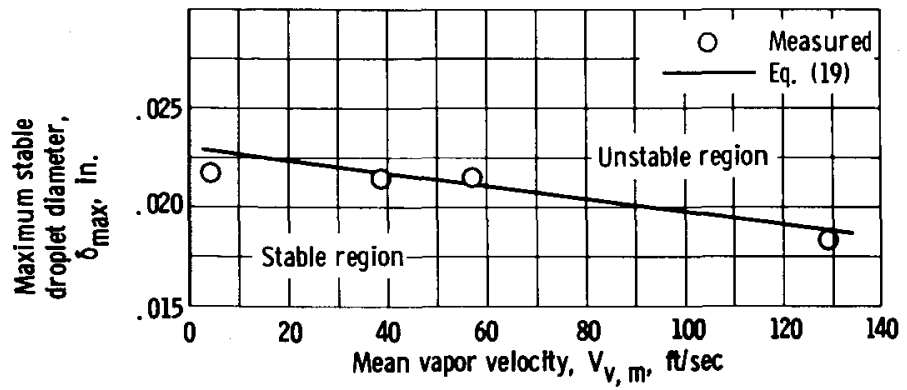

Figure 18. - Maximum stable droplet diameter as function of mean vapor velocity. given vapor quality condition, certain droplets, which exceed a diameter of the most frequent droplet (see table VI), were moving stably close to a condenser wall with no oscillation due to turbulence. The droplets that obeyed the stability in flow and belonged to this size group were termed "maximum stable droplets". Therefore, in order to get a good statistical average diameter of a maximum stable droplet, 12 size measurements along the film were made of this category of droplets. The data obtained at various vapor velocities and quality conditions are tabulated in table VI, and the results are plotted as a function of a mean vapor velocity in figure 18 . The straight-line equation

$$
\delta_{\max }=0.0230-3.1 \times 10^{-5} \mathrm{~V}_{\mathrm{v}, \mathrm{m}}
$$

fits the measured data within an accuracy of $2.5 \mathrm{rms}$ percent. The

diameter of a maximum stable droplet size decreases with an increase in a mean vapor velocity. For example, at the vapor velocity of 4 feet per second, $\delta_{\max }=0.0218$ inch, and at vapor velocity of 129 feet per second, $\delta_{\max }=0.0182 \mathrm{inch}$.

Equation (19) defines not the upper and not the lower limit of a stable droplet size, but it characterizes the statistical average obtained as a value of the root mean square. A knowledge of the maximum stable droplet size in a condenser tube is useful for several reasons. First, the maximum stabile dropiet size ${ }^{0}{ }_{\max }$ can itseî́ be usec in piace of́ an average droplet size for making conservative estimates of mass transfer rates. Second, under some conditions, it may be possible to relate a given droplet size to maximum droplet size in order to predict tube slugging in a small diameter condenser tube.

Critical droplet diameter in ground tests. - The critical droplet diameter was measured from the droplet track left on a grayish condenser wall, such as shown in figure 19 . Because of a small magnification factor of films $(\times 1.40$ to $\times 2.20)$, the critical droplet diameter was not observed in the airplane tests. Measurement of the critical droplet 


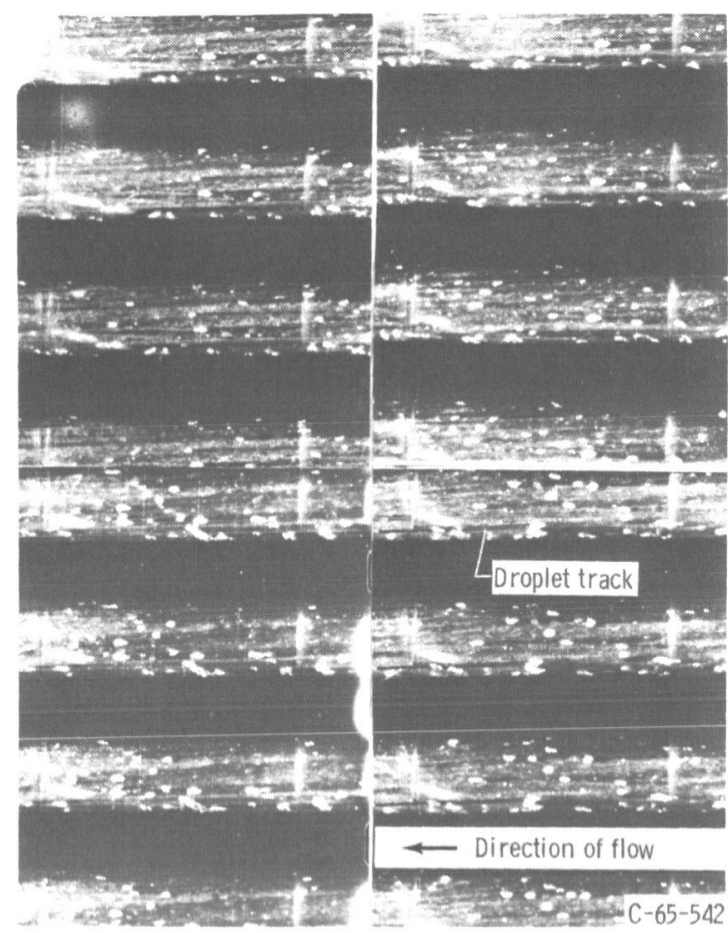

Figure 19. - Dropwise condensation in glass tube. Tracks left by moving droplets of variable width and equivalent to maximum droplet contact diameter. $\quad$ X8.9.

diameter at its incipient movement position was accomplished with the aid of crosshair micrometer dials with which the motion analyzer was equipped. For each vapor quality condition, from three to six such measurements were made along the film being analyzed. From these measurements, the average critical droplet diameter was determined as the root-meansquare value. The data obtained are listed in table VI, and the results are plotted as a function of vapor quality $\mathrm{x}$ in figure 20. As shown in the figure, the critical droplet diameter on a condenser wall is strongly dependent on the vapor phase quality. For example, for a vapor quality of 0.82 , the critical droplet diameter is $0.0051 \mathrm{inch}$, and for a vapor quality of 0.03 , the critical droplet diameter is 0.0120 inch.

The measured average values of the critical droplet diameter on a condenser wall were correlated with the theoretical values obtained by solving the force balance equation:

$$
\delta_{\mathrm{c}}=\left(\frac{\mathscr{B}-\mathscr{C}}{\mathscr{A}}\right)^{3 / 2}
$$

The derivation of this equation is given in appendix D.

The correlation of data, with the exception of droplets very close to the vapor-liquid interface, is fairly good. For example, at a vapor quality of 0.82 , the correlation is within 3.0 percent, at 0.36 , the correlation is within 4.0 percent, and at 0.20 and 0.03 , the correlation deviates to approximately 15.0 percent. On the average, the theoretical equation (eq. (20)) correlates with the measured data points within $9 \mathrm{rms}$ percent.

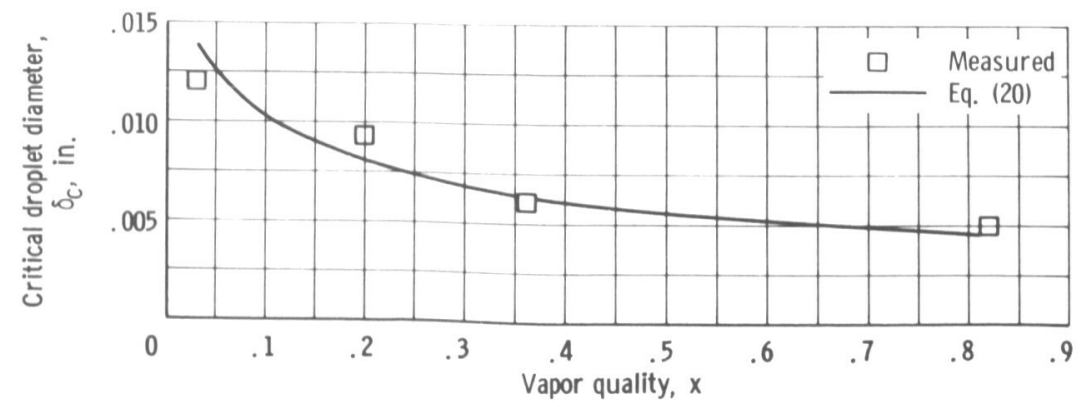

Figure 20. - Critical droplet diameter as function of vapor quality. 


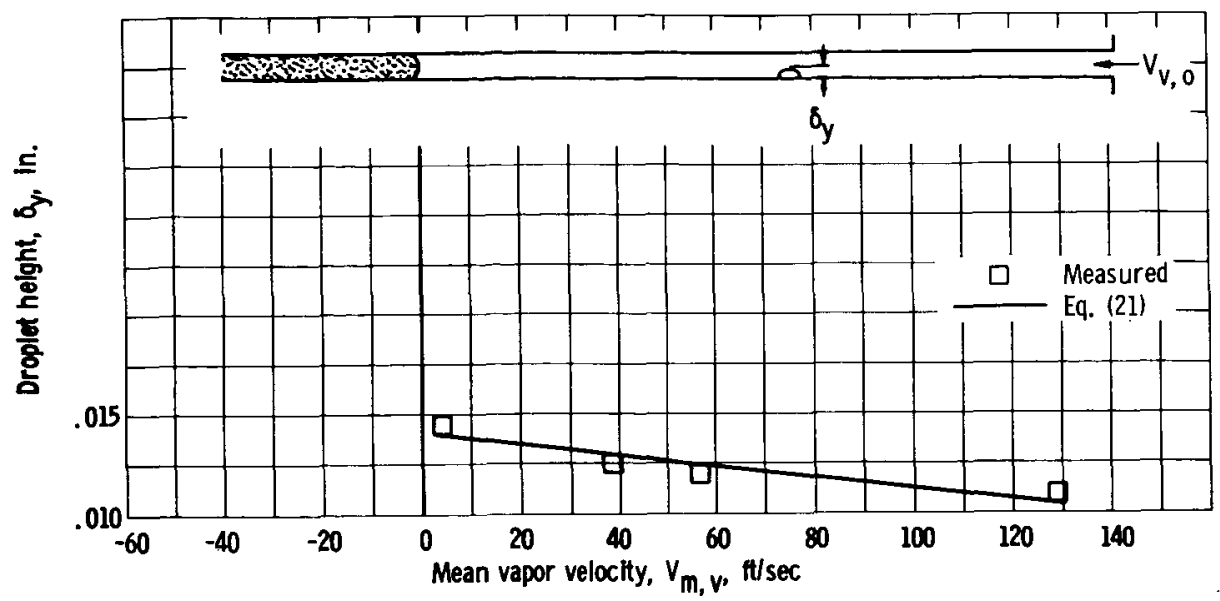

Figure 21. - Droplet height as function of vapor velocity.

Droplet height on condenser wall in ground tests. - In a study of frictional pressure drop of the vapor phase in turbulent flow through pipes, slowly moving droplets on a condenser wall may be regarded as the spherical segments that increase the effective roughness of the condenser tube wall. Therefore, the measured droplet height on a condenser wall may be regarded as an important roughness parameter $\delta_{\mathrm{y}} / \mathrm{R}$, the so-called relative roughness introduced by Nikuradse in reference 8 . In this case, the droplet height $\delta_{y}$ is regarded as a protrusion and $R$ denotes the hydraulic radius of the cross section. From considerations of similtude it may be concluded that, in this case, the resistance coefficient depends on the relative roughness only, which is the determining factor of the frictional pressure drop in the boundary layer.

In the size range of the most frequent droplet, 12 measurements were made of the droplet height $\delta_{\mathrm{y}}$ on a condenser wall. For a given vapor velocity and quality condition, the mean droplet height was taken as a value of the root mean square. The data, thus obtained, are tabulated in table VI, and the results are plotted in figure 21 as a function of mean vapor velocity. The straight line, expressed by the following relation

$$
\delta_{\mathrm{y}}=0.0140-0.269 \times 10^{-4} \mathrm{v}_{\mathrm{v}, \mathrm{m}}
$$

fits the plotted data points within an accuracy of $1.30 \mathrm{rms}$ percent.

\section{CONCLUDING REMARKS}

The dominating factor in this analysis is the definition of the mercury droplet size distribution function in a uniformly cooled ( $q / A=$ constant), glass condenser tube in $1-\mathrm{g}$ and zero-gravity environments. The observed droplet diameter and droplet frequencies followed the gamma distribution law, which expresses the fraction of droplets that have a 
certain diameter. The proposed model of droplet size distribution at various vapor velocities and quality conditions along the condenser tube proved to be quite successful, and $1.72 \mathrm{rms}$ percent agreement between the measured and calculated values was obtained. A correction factor was obtained to allow estimation of droplet distribution in a zerogravity environment from data obtained in a $1-\mathrm{g}$ environment. It is anticipated that this analysis will furnish a convenient starting point for quantitative statistical description of droplet size distribution in a uniformly cooled condenser tube.

The critical droplet diameter on a condenser tube wall was defined by a theoretical expression derived from the force balance equation, which correlated with the measured values within an accuracy of $9 \mathrm{rms}$ percent. Also, the measured values of the height of the most frequent droplet on a condenser wall and the diameter of the maximum stable droplet in turbulent vapor stream, at various vapor velocities and qualities, were defined by the best fit equations, which gave 1.30 and $2.50 \mathrm{rms}$ percent deviation from the measured values, respectively. The maximum stable droplet size in a turbulent vapor flow, and also the height of a most frequent droplet on a condenser wall was expressed as a function of a mean vapor velocity.

It was also observed that for a given flow condition, the droplets were more uniformly dispersed throughout the vapor phase and on the condenser tube wall in a zero-gravity environment than in a $1-\mathrm{g}$ environment. In addition to this, droplets moved with a more uniform axial velocity (less turbulent) in zero gravity than in a $1-\mathrm{g}$ environment.

\section{Lewis Research Center,}

National Aeronautics and Space Administration,

Cleveland, Ohio, September 28, 1966, 701-04-00-02-22. 


\section{APPENDIX A}

\section{SYMBOLS}

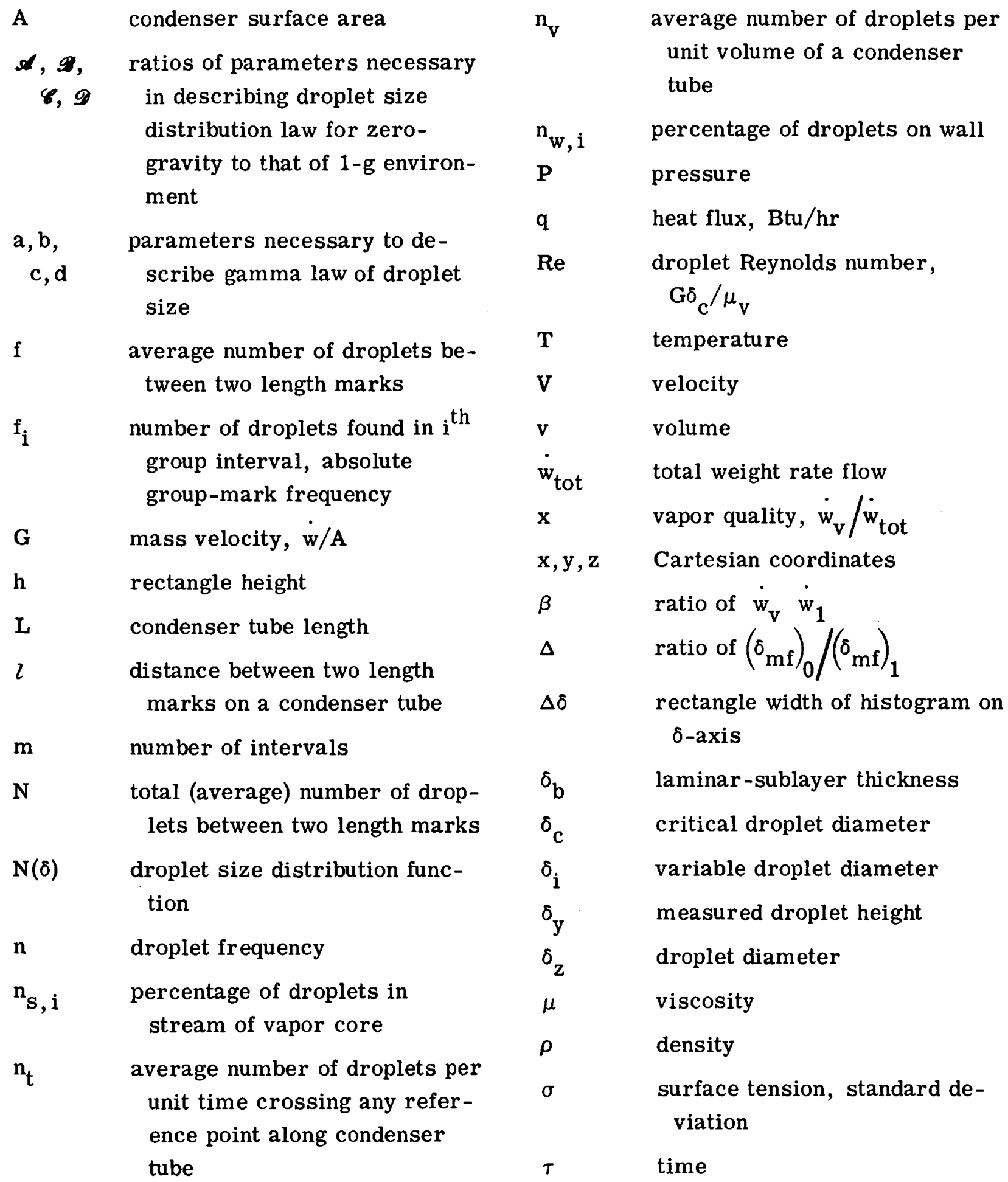




\begin{tabular}{|c|c|c|c|}
\hline \multirow[t]{2}{*}{$\Delta \tau$} & $\begin{array}{l}\text { time interval for droplet to travel } \\
\text { between two length marks, } \\
\text { Number of frames recorded }\end{array}$ & $\mathbf{s}$ & $\begin{array}{l}\text { liquid } \\
\text { stream of vapor core }\end{array}$ \\
\hline & Frame rate , sec & sat & saturation \\
\hline \multirow[t]{3}{*}{$\varphi$} & droplet flux, average number of & $\mathrm{sh}$ & superheat \\
\hline & droplets crossing per unit area & $\mathrm{t}$ & time \\
\hline & per unit time & tot & total \\
\hline \multicolumn{2}{|c|}{ Subscripts: } & $\mathrm{v}$ & vapor, volume \\
\hline act & actual & $\mathbf{w}$ & wall \\
\hline c & critical & $\mathrm{y}$ & height \\
\hline i & $\mathrm{i}^{\text {th }}$ interval & $\mathbf{z}$ & diameter \\
\hline $\mathrm{m}$ & mean & $\delta$ & droplet \\
\hline $\mathrm{mf}$ & most frequent & 0 & zero-gravity environment \\
\hline $\max$ & maximum & 1 & $1-g$ environment \\
\hline
\end{tabular}




\section{APPENDIX B}

\section{DROPLET SIZE DISTRIBUTION FUNCTION}

From the measured data, both the droplet diameters $\delta$ and droplet frequencies $\mathbf{n}$ followed the gamma distribution law. The gamma probability density function is given in reference 6 by

$$
f(\delta)=\frac{\lambda}{\Gamma(r)}(\lambda \delta)^{r-1} e^{-\lambda \delta} \quad \delta>0
$$

where $r$ and $\lambda$ are positive constants.

An area of the rectangle of the histogram which represents the relative frequency is given by $\mathrm{n} \Delta \delta$. Therefore, multiplying equation (B1) by this factor results in

$$
\mathrm{f}(\delta) \mathrm{n} \Delta \delta=\mathrm{n} \Delta \delta \frac{\lambda}{\Gamma(\mathrm{r})}(\lambda \delta)^{\mathrm{r}-1} \mathrm{e}^{-\lambda \delta}
$$

Letting

$$
\begin{gathered}
\mathrm{N}(\delta)=\mathrm{f}(\delta) \mathrm{n} \Delta \delta \\
\mathrm{c}=\mathrm{n} \Delta \delta \frac{\lambda}{\Gamma(\mathrm{r})} \\
\mathrm{a}=\lambda
\end{gathered}
$$

and

$$
b=(r-1)
$$

gives equation (B1) in the form

$$
\mathrm{N}(\delta)=c(\mathrm{a} \delta)^{\mathrm{b}} \mathrm{e}^{-\mathrm{a} \delta}
$$

where $a, b$, and $c$ are the three characteristic parameters that make a frequency function $\mathrm{N}(\delta)$ to be determined from the measured data.

The relation among the three characteristic parameters in equation (B2) was determined from the following two imposed conditions: 
(1) By definition, the total area under the distribution curve defining the frequency function $N(\delta)$ is equal to 1 . Thus,

$$
\int_{0}^{\infty} \mathrm{N}(\delta) \mathrm{d} \delta=1
$$

Even though the droplet size has been measured from $\delta=0$ to $\delta=\delta_{\max }$, the largest droplet size found, infinity as the upper limit of the integral was used in equation (B3) in order to simplify the calculations. The use of this limit does not introduce any appreciable error, since no excessive large droplets exist, and the distribution function $\mathrm{N}(\delta)$ must approach zero very rapidly for $\delta>\delta_{\max }$

Substituting equation (B2) into equation (B3) yields

$$
\int_{0}^{\infty} \mathrm{c}(\mathrm{a} \delta) \mathrm{e}^{-\mathrm{a} \delta} \mathrm{d} \delta=1
$$

Making the substitution $\mathrm{x}=\mathrm{a} \delta$ into equation (B4) gives

$$
\int_{0}^{\infty} x^{b} e^{-x} d x=\frac{a}{c}
$$

The integral may be evaluated by the gamma function thus giving the relation among the three parameters from the first imposed condition

$$
\frac{\mathrm{a}}{\mathrm{c}}=\Gamma(\mathrm{b}+1)
$$

(2) The gamma distribution curve exhibits a maximum that may be located by differentiating equation (B2) with respect to $\delta$ and setting the result equal to zero. Thus,

$$
\frac{d}{d \delta} N(\delta)=0=-a c(a \delta)^{b} e^{-a \delta}+a b c(a \delta)^{b-1} e^{-a \delta}
$$

Solving for ${ }^{\delta} \mathrm{mf}$ gives

$$
\delta_{\mathrm{mf}}=\frac{\mathrm{b}}{\mathrm{a}}
$$


The relation of the third parameter of the normal distribution function was obtained by substituting equation (B7) into equation (B6) and eliminating the parameter a. Thus,

$$
c=\frac{b}{\delta_{m f}} \frac{1}{\Gamma(b+1)}
$$

The droplet diameter $\delta_{\mathrm{mf}}$ is determined from the gamma distribution function, the frequency of occurrence of which is the maximum. The experimental value of $\delta_{\mathrm{mf}}$ is known.

Substitution of equations (B7) and (B8) into equation (B2) eliminates the parameters $\mathrm{a}$ and $\mathrm{c}$, which gives the maximum value $\mathrm{N}\left(\delta_{\mathrm{mf}}\right)$ of the gamma distribution function. Thus,

$$
\mathrm{N}\left(\delta_{\mathrm{mf}}\right) \delta_{\mathrm{mf}}=\frac{\mathrm{b}^{\mathrm{b}+1}}{\Gamma(\mathrm{b}+1)} \mathrm{e}^{-\mathrm{b}}
$$

Since equation (B9) is a transcendental function in $\mathrm{b}, \mathrm{N}\left(\delta_{\mathrm{mf}}\right) \delta_{\mathrm{mf}}$ was plotted as a function of $\mathrm{b}$, and for each value of $\mathrm{N}\left(\delta_{\mathrm{mf}}\right) \delta_{\mathrm{mf}}$ obtained from the measurements, the parameter $b$ was obtained from this plot. The numerical values of the parameters a and $c$ were calculated from equations (B7) and (B8), respectively. 


\section{APPENDIX C}

\section{VAPOR VELOCITY DISTRIBUTION ALONG CONDENSER TUBE}

If $\mathrm{q} / \mathrm{A}$ is constant, as it will be for condensing vapors, it is reasonable to assume that a mean vapor velocity will have a linear distribution along the condenser length. Thus,

$$
v_{v, m}=v_{v, o}\left(1-\frac{z}{L}\right)
$$

Consider the condenser tube at the region of inlet, as shown in figure 22 , where $\mathrm{P}_{4}$ is the measured pressure, equal to 18.30 pounds per square inch absolute, $P_{6}$ is the inlet pressure, equal to $0.90 \mathrm{P}_{4}, \mathrm{~T}_{\text {sat }}=685^{\circ} \mathrm{F}$ or $1145^{\circ} \mathrm{R}$, which corresponds to $\mathrm{P}_{6}, \mathrm{~T}_{\text {act }}=$ $875^{\circ} \mathrm{F}$ or $1335^{\circ} \mathrm{R}$ measured temperature, the vapor quality is 90 percent, $\rho_{\text {sat }}$ is 0.270 pound per cubic foot, which corresponds to $P_{6}$, and $\rho_{\text {sat }}=0.270 \frac{1145}{1335}=0.232$ pound per cubic foot.

For a constant diameter tube, the vapor velocity may be defined by

$$
\mathrm{v}_{\mathrm{v}}=\frac{\mathrm{x} \dot{\mathrm{w}}}{\rho_{\mathrm{v}} \mathrm{A}}
$$

Thus, the vapor velocity corresponding to saturation conditions will be

$$
\left(\mathrm{v}_{\mathrm{v}}\right)_{\mathrm{sat}}=\frac{0.090(0.0385)(144)}{0.270 \frac{\pi}{4}(0.440)^{2}}=\frac{5.000}{0.0412}=121 \mathrm{ft} / \mathrm{sec}
$$

and the inlet vapor velocity corrected due to superheat results in

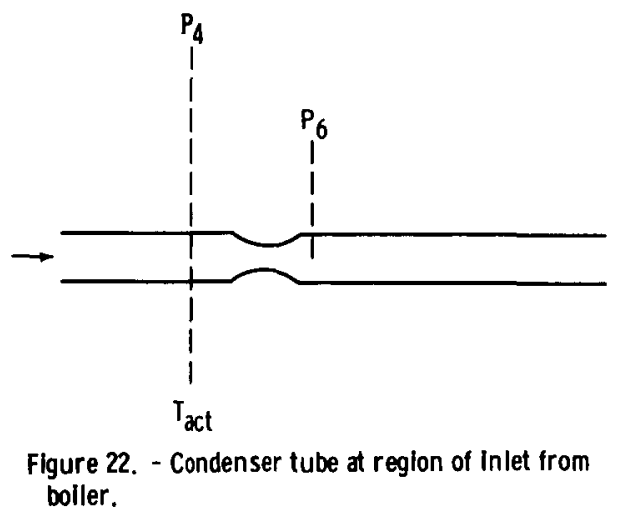

boller.

$$
\left(\mathrm{v}_{\mathrm{v}, \mathrm{o}}\right)_{\mathrm{sh}}=\frac{0.270}{0.232} 121=141 \mathrm{ft} / \mathrm{sec}
$$

Substituting this value of $\left(\mathrm{V}_{\mathrm{v}, \mathrm{o}}\right)_{\mathrm{sh}}$ into equation (C1) results in

$$
\mathrm{v}_{\mathrm{v}, \mathrm{m}}=141\left(1-\frac{\mathrm{z}}{\mathrm{L}}\right)
$$




\section{APPENDIX D}

\section{CRITICAL DROPLET SIZE}

Consider a microscopic droplet resting on a solid surface under the influence of static forces, gravity, and drag, as illustrated in figure 23. The retarding force that re-

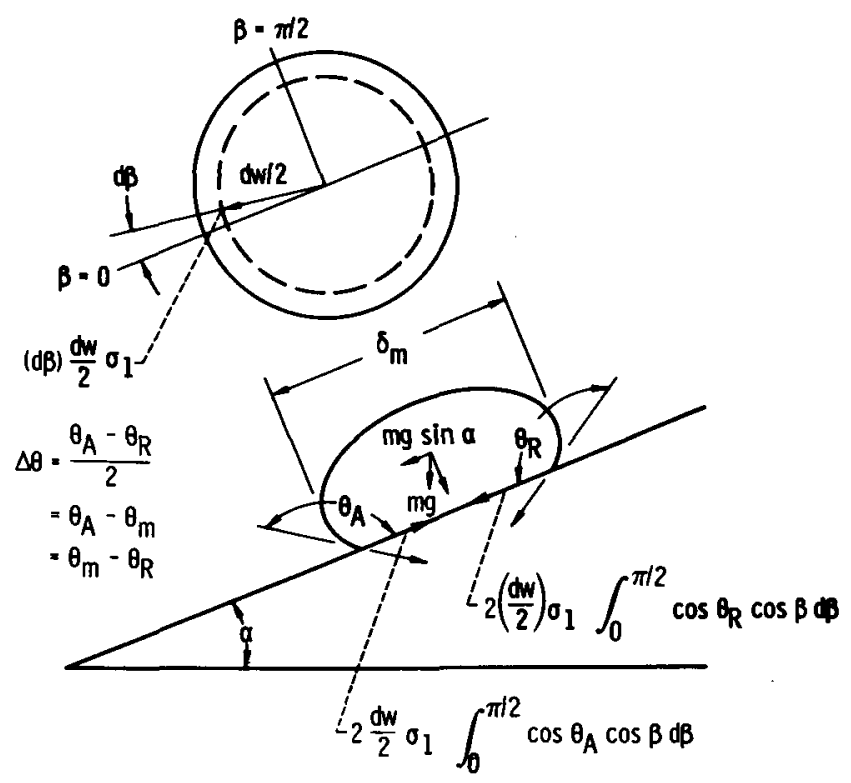

Figure 23. - Vector analysis of forces acting on droplet at moment of incipient movement. sists droplet movement is attributed to adhesion force (attraction force exerted between the molecules of liquid droplet and the molecules of solid surface). It is assumed that, for a small droplet, adhesion tension forces at the juncture of the liquid-solid surface will keep the droplet stationary on the tube wall in spite of the drag and gravity forces on the droplet. If droplet size increases above a critical size, the drag and gravity forces will exceed the resisting adhesion tension forces at the wall, and the droplet will begin to move. The shape of a stationary droplet is assumed to be unaffected by the drag or gravity forces. Thus,

$$
\sum F=0
$$

\section{Adhesion force \pm Gravity force - Drag force $=0$}

Changing the signs in the preceding equation gives

$$
\text { -Adhesion force } \pm \text { Gravity force }+ \text { Drag force }=0
$$

From figure 23 , the equation of motion is

$-2 \mathrm{~K}_{1} \frac{\mathrm{d}_{\mathrm{w}}}{2} \sigma_{l}\left(\cos \theta_{\mathrm{R}}+\cos \theta_{\mathrm{A}}\right) \int_{0}^{\pi / 2} \cos \beta \mathrm{d} \beta \pm \mathrm{K}_{2} \mathrm{v}_{\delta} \frac{\rho_{l}}{\mathrm{~g}_{\mathrm{c}}} \mathrm{g} \sin \alpha+\mathrm{K}_{3} \mathrm{~A}_{\delta} \frac{\rho_{\mathrm{v}} \mathrm{V}_{\mathrm{v}}}{2 \mathrm{~g}_{\mathrm{c}}} \mathrm{C}_{\mathrm{D}}=0$ 
where

g acceleration due to gravity

$g_{c} \quad$ conversion factor

$\mathrm{C}_{\mathrm{D}}$ drag coefficient on droplet

$\alpha \quad$ surface inclination relative to horizontal

Substituting the following trigonometric expressions

$$
\begin{aligned}
& { }^{\theta_{\mathrm{A}}}=\theta_{\mathrm{m}}+\Delta \theta \\
& \theta_{\mathrm{R}}=\theta_{\mathrm{m}}-\Delta \theta
\end{aligned}
$$

and

$$
\cos \theta_{\mathbf{R}}+\cos \theta_{\mathbf{A}}=2 \cos \theta_{\mathrm{m}} \cos \Delta \theta
$$

into equation (D1) and integrating result in

$$
-2 \mathrm{~d}_{\mathrm{w}} \sigma_{l} \cos \theta_{\mathrm{m}} \cos \Delta \theta \pm \mathrm{K}_{2} \mathrm{~V}_{\delta} \frac{\rho_{l}}{\mathrm{~g}_{l}} \mathrm{~g} \sin \alpha+\mathrm{K}_{3} \mathrm{~A}_{\delta} \frac{\rho_{\mathrm{v}} \mathrm{V}_{\mathrm{v}}}{2 \mathrm{~g}_{l}} \mathrm{C}_{\mathrm{D}}=\mathbf{0}
$$

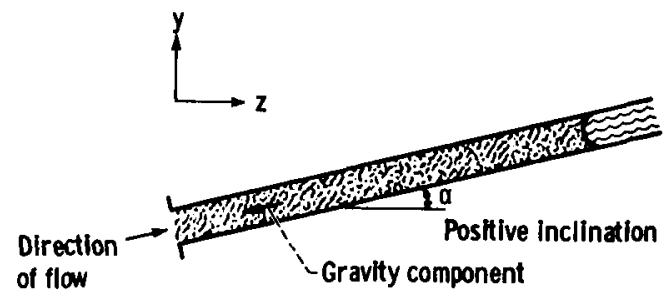

(a) Vapor flows against gravity.

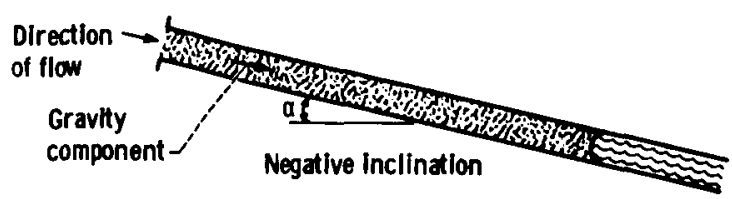

(b) Vapor flows in direction of gravity.

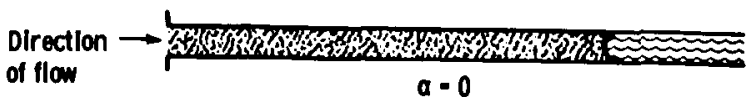

(c) Gravity has negligible effect when $a \rightarrow 0$ or $g / g_{c} \rightarrow 0$.
Equation (D2), with respective signs, applies for all three cases shown in figure 24. In this analysis, for a horizontal tube when $\alpha=0$, the gravity term drops out, and equation (D2) reduces to the following form:

$$
\begin{aligned}
-2 \mathrm{~K}_{1} \sigma_{l} \cos \theta_{\mathrm{m}} & \cos \Delta \theta \\
& +\mathrm{K}_{3} \mathrm{~A}_{\delta} \frac{\rho_{\mathrm{v}} \mathrm{V}_{\mathrm{v}}}{2 \mathrm{~g}_{\mathrm{c}}} \mathrm{C}_{\mathrm{D}}=0
\end{aligned}
$$

The quantities $\mathrm{K}_{1}, \mathrm{~K}_{2}$, and $\mathrm{K}_{3}$ in equation (D2) are the shape factors of a droplet distorted by the curvature of the tube wall defined as follows: 
Perimeter shape factor of a droplet contact with tube wall:

$$
\mathrm{K}_{1}=\frac{\text { Measured perimeter of droplet contact with tube wall }}{\text { Apparent perimeter of droplet contact with tube wall }}
$$

Volume shape factor:

$$
\mathrm{K}_{2}=\frac{\text { Volume of spherical segment having contact angle of } 110^{\circ} \text { approximately }}{\text { Volume of equivalent spherical droplet }}
$$

Cross-sectional area shape factor:

$$
\mathrm{K}_{3}=\frac{\text { Cross-sectional area of droplet on glass plate }}{\text { Cross-sectional area of equivalent droplet in glass tube }}
$$

In order to obtain statistical averages for $\mathrm{K}_{1}, \mathrm{~K}_{2}$, and $\mathrm{K}_{3}, 12$ mercury droplets ranging in diameter from 0.0051 to 0.0120 inch were photographed on a glass plate and in a glass tube. From enlarged images of a droplet (side view, bottom view, and top view), the shape factors were determined statistically from the measured droplet dimensions.

Using the following numerical values of the shape factors,

$$
\begin{aligned}
& K_{1}=1.23 \\
& K_{2}=0.832 \\
& K_{3}=0.875
\end{aligned}
$$

and the following properties of mercury at the temperature of saturation of $680^{\circ} \mathrm{F}$, from reference 9 ,

$$
\begin{aligned}
\rho_{l} & =794 \mathrm{lb} / \mathrm{cu} \mathrm{ft} \\
\rho_{\mathrm{v}} & =0.260 \mathrm{lb} / \mathrm{cu} \mathrm{ft} \\
\mu_{\mathrm{v}} & =0.148 \mathrm{lb} / \mathrm{ft} \mathrm{hr} \\
\sigma_{l} & =0.027 \mathrm{lb} / \mathrm{hr}
\end{aligned}
$$


and

$$
\mathrm{v}_{\mathrm{v}}^{2}=\frac{\mathrm{G}^{2}}{\rho_{\mathrm{v}}^{2}} \mathrm{x}^{2}
$$

where $G=\dot{w} / A=131300$ pounds per square foot per hour, and $d_{w}$ is approximately equal to $\delta_{\mathrm{m}}$ gives equation (D3) in the form

$$
-19.880 \delta_{\mathrm{c}}^{2} \frac{\mathrm{G}^{2}}{\mathrm{~g}_{\mathrm{c}}} \mathrm{x}^{2} \mathrm{C}_{\mathrm{D}}+\delta_{l} \cos \theta_{\mathrm{m}} \cos \Delta \theta=0
$$

Evaluation of drag coefficient. - In the critical droplet size range investigated $\left(0.0051 \leq \delta_{\mathrm{m}} \leq 0.0120\right.$ in. $)$ it may be safe to assume that the critical droplet formed on a condenser wall will be within the thickness of a laminar sublayer. This assumption is based on the laminar sublayer theory in turbulent flow developed by Eckert in reference 4.

A well-known expression for the drag coefficient $C_{D}$ is given in reference 10 . In the laminar sublayer, the drag coefficient may be expressed by

$$
C_{D}=\frac{24}{\operatorname{Re}}+\frac{4.8}{\operatorname{Re}^{1 / 3}}
$$

Defining the Reynolds number of a critical droplet size by

$$
\operatorname{Re}=\frac{G{ }^{\delta} \mathbf{x}}{\mu_{v}}
$$

gives the drag coefficient:

$$
C_{D}=\frac{24 \mu_{v}}{G \delta c_{c}}+\frac{4.8 \mu_{v}^{1 / 3}}{G^{1 / 3} \delta_{c}^{1 / 3} x^{1 / 3}}
$$

Substituting this expression for $C_{D}$, the numerical value of the conversion factor

$$
\mathrm{g}_{\mathrm{c}}=4.17 \times 10^{8} \frac{(\mathrm{lb} \text { mass })(\mathrm{ft})}{(\mathrm{lb} \text { force })\left(\mathrm{hr}^{2}\right)}
$$


and

$$
\mu_{\mathrm{v}}=0.148 \frac{\mathrm{lb} \text { mass }}{(\mathrm{ft})(\mathrm{hr})}
$$

into equation (D4) and simplifying result in

$$
\left(0.945 \times 10^{-8} \mathrm{G}^{5 / 3} \mathrm{x}^{5 / 3} \delta_{\mathrm{c}}^{5 / 3}+16.934 \times 10^{-8} \mathrm{Gx}-\cos \theta_{\mathrm{m}} \Delta \theta\right) \delta_{\mathrm{c}}=0
$$

Equation (D4) is the force-balance equation of a mercury droplet at its incipient movement in a horizontal glass tube. Solving equation (D4) for the droplet diameter gives an expression for the critical droplet size $\delta_{\mathrm{c}}$

$$
\delta_{\mathrm{c}}=\left(\frac{\mathscr{D}-\mathscr{C}}{\mathscr{A}}\right)^{3 / 2}
$$

where

$$
\begin{gathered}
\mathscr{\mathscr { C }}=0.945 \times 10^{-8} \mathrm{G}^{5 / 3} \mathrm{x}^{5 / 3} \\
\mathscr{D}=\cos \theta_{\mathrm{m}} \cos \Delta \theta \\
\mathscr{C}=16.934 \times 10^{-8} \mathrm{Gx}
\end{gathered}
$$

The following inputs obtained from the measurements

$$
\begin{gathered}
{ }_{\mathrm{m}}=110^{\circ} \\
\Delta \theta=10^{\circ} \\
\mathrm{G}=131300 \mathrm{lb} /(\mathrm{sq} \mathrm{ft})(\mathrm{hr})
\end{gathered}
$$

were used to solve equation (D5) in the range of vapor quality analyzed $(0.010 \leq x$ $\leq 0.900$ ) by the use of a digital computer. 


\section{REFERENCES}

1. Koestel, Alfred; and Gido, R. G.: Mercury Wetting and Non-wetting Condensing Research. Rep. No. ER-5214, Thompson Ramo Wooldridge, Inc., Jan. 1963.

2. Heller, J. A.: Sunflower I Condenser-Subcooler. Rep. No. ER-5088 (NASA CR54013), Thompson Ramo Wooldridge, Inc., June 1, 1964.

3. Albers, James A.; and Macosko, Robert P.: Experimental Pressure-Drop Investigation of Nonwetting, Condensing Flow of Mercury Vapor in a Constant-Diameter Tube in 1-G and Zero-Gravity Environments. NASA TN D-2838, 1965.

4. Eckert, E. R. G.: Introduction to Heat and Mass Transfer. McGraw-Hill Book Co., Inc., 1963.

5. Hoel, Paul G.: Introduction to Mathematical Statistics. Second ed., John Wiley and Sons, Inc., 1954.

6. Parzen, Emanuel: Stochastic Processes. Holden-Day, Inc., San Francisco, 1962.

7. Marquardt, Donald W.: An Algorithm for Least-Squares Estimation of Nonlinear Parameters. Soc. Indust. Appl. Math. J., vol. 11, no. 2, June 1963, pp. 431-441.

8. Schlichting, Hermann (J. Kestin, trans.): Boundary Layer Theory. McGraw Hill Book Co., Inc., 1955.

9. Weatherford, W. D., Jr.; Tyler, John C.; and Ku, P. M.: Properties of Inorganic Energy-Conversion and Heat-Transfer Fluids for Space Applications. (AF WADDTR-61-96), Southwest Research Institute, Nov. 1961.

10. Dussourd, Jules L.: A Theoretical and Experimental Investigation of a Deceleration Probe for the Measurement of Several Properties of a Droplet-Laden Air Stream. Mass. Inst. of Tech., PhD Thesis, Oct. 1954. 
Lewis motion picture C-221 is available on loan. Requests will be filled in the order received. You will be notified of the approximate date scheduled.

The film (16 mm, $12 \mathrm{~min}$, color, sound) includes an introduction to space power systems, a description of the MECA - AJ-2 flight package and associated hardware, and high-speed film sequences of mercury condensing in 1-g and zero-gravity environments.

Lewis motion picture $\mathrm{C}-221$ is available on request to:

Chief, Technical Information Division (5-5)

National Aeronautics and Space Administration

Lewis Research Center

21000 Brookpark Road

Cleveland, Ohio 44135

CUT

Date

Please scnd, on loan, copy of Iewis film C-221

Name of Organization

Street Number

City and State

Zip code Attention: $\mathrm{Mr}$.

Title 

stamp here

Chief, Technical Information Division National Aeronautics and Space Administration Lewis Research Center

21000 Brookpark Road

Cleveland, Ohio 44135 
The aeronawical and sace activities of the United States shall be condacted so as to contribute, to the expansion of buman knowledge of phenomena is the atmosphere and space. The Administration sball provide for the thidest practicable and appropriate dissemination of information concerning its actitities and the results thereof."

TNATIONAT AERONAUTICS AND SPACE ACT OF 1958

\section{NASA SCIENTIFIC AND TECHNICAL PUBLICATIONS}

TECHNICAL REPORTS: Scientific and technical information considered important, complete, and a lasting contribution to existing knowledge.

TECHNICAL NOTES: Information less broad in scope but nevertheless of Importance as a contribution to existing knowledge.

TECHNICAL MEMORANDUMS: Information receiving limited distribution because of preliminary data, security classification, or other reasons.

CONTRACTOR REPORTS. Technical information generated in connection with a NASA contract or grant and released under NASA auspices.

TECHNICAL TRANSLATIONS: Information published in a foreign language considered to merit NASA distribution in English.

TECHNICAL REPRINTS: Information derived from NASA activities and initially published in the form of journal articles.

SPECIAL PUBLICATIONS: Information derived from or of value to NASA activities but not necessarily reporting the results of individual NASA-programmed scientific efforts. Publications include conference proceedings, monographs, data compilations, handbooks, sourcebooks, and special bibliographies.

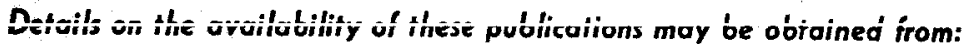

SCIENTIFIC AND TECHNICAL INFORMATION DIVISION

NATIONAL AERONAUTICS AND SPACE ADMINISTRATION

Woshington, D.C. 20546 